\title{
Sample Path Properties of Anisotropic Gaussian Random Fields
}

\author{
Yimin Xiao * \\ Michigan State University
}

February 15, 2008

\begin{abstract}
Anisotropic Gaussian random fields arise in probability theory and in various applications. Typical examples are fractional Brownian sheets, operator-scaling Gaussian fields with stationary increments, and the solution to the stochastic heat equation.

This paper is concerned with sample path properties of anisotropic Gaussian random fields in general. Let $X=\left\{X(t), t \in \mathbb{R}^{N}\right\}$ be a Gaussian random field with values in $\mathbb{R}^{d}$ and with parameters $H_{1}, \ldots, H_{N}$. Our goal is to characterize the anisotropic nature of $X$ in terms of its parameters explicitly.

Under some general conditions, we establish results on the modulus of continuity, small ball probabilities, fractal dimensions, hitting probabilities and local times of anisotropic Gaussian random fields. An important tool for our study is the various forms of strong local nondeterminism.
\end{abstract}

Running head: Sample Path Properties of Anisotropic Gaussian Fields

2000 AMS Classification numbers: 60G15, 60G17; 60G60; 42B10; 43A46; $28 \mathrm{~A} 80$.

Key words: Anisotropic Gaussian fields, fractional Brownian sheet, stochastic heat equation, stochastic wave equation, sectorial local nondeterminism, strong local nondeterminism, modulus of continuity, small ball probability, Hausdorff dimension, local times, joint continuity.

\section{Contents}

1 Introduction $\quad 2$

2 Examples and general assumptions $\quad \mathbf{5}$

2.1 Fractional Brownian sheets . . . . . . . . . . . . . . . . . . . . . 5

2.2 Anisotropic Gaussian random fields with stationary increments . . . . . . . . . . . . . 7

2.3 Solutions to stochastic partial differential equations . . . . . . . . . . . . . . . . . 8

2.4 General assumptions . . . . . . . . . . . . . . . . . . . . . . . . . . 12

3 Properties of strong local nondeterminism $\quad 14$

4 Modulus of continuity $\quad 18$

${ }^{*}$ Research partially supported by the NSF grant DMS-0404729. 


\section{Introduction}

Gaussian random fields have been extensively studied in probability theory and applied in a wide range of scientific areas including physics, engineering, hydrology, biology, economics and finance. Two of the most important Gaussian random fields are respectively the Brownian sheet and fractional Brownian motion.

The Brownian sheet $W=\left\{W(t), t \in \mathbb{R}_{+}^{N}\right\}$, which was first introduced by a statistician J. Kitagawa in 1951, is a centered Gaussian random field with values in $\mathbb{R}^{d}$ and covariance function given by

$$
\mathbb{E}\left[W_{i}(s) W_{j}(t)\right]=\delta_{i j} \prod_{k=1}^{N} s_{k} \wedge t_{k}, \quad \forall s, t \in \mathbb{R}_{+}^{N},
$$

where $\delta_{i j}=1$ if $i=j$ and 0 if $i \neq j$. When $N=1, W$ is the ordinary Brownian motion in $\mathbb{R}^{d}$. For $N \geq 2, W$ has independent increments over disjoint intervals in $\mathbb{R}_{+}^{N}$ and such increments are stationary. We refer to Adler (1981) and Khoshnevisan (2002) for systematic accounts on properties of the Brownian sheet and to Walsh (1986) and the articles in this volume for its important roles in stochastic partial differential equations.

For a fixed constant $0<\alpha<1$, an $(N, d)$-fractional Brownian motion with index $\alpha$ is a centered Gaussian random field $X^{\alpha}=\left\{X^{\alpha}(t), t \in \mathbb{R}^{N}\right\}$ with values in $\mathbb{R}^{d}$ and covariance function given by

$$
\mathbb{E}\left[X_{i}^{\alpha}(s) X_{j}^{\alpha}(t)\right]=\frac{1}{2} \delta_{i j}\left(|s|^{2 \alpha}+|t|^{2 \alpha}-|s-t|^{2 \alpha}\right), \quad \forall s, t \in \mathbb{R}^{N},
$$

where $|\cdot|$ denotes the Euclidean norm in $\mathbb{R}^{N}$. The existence of $X^{\alpha}$ follows from the positive semidefiniteness of the kernel on the right hand side of (1.2); see Samorodnitsky and Taqqu (1994) for a proof. When $N=1$ and $\alpha=1 / 2, X^{\alpha}$ is again the Brownian motion in $\mathbb{R}^{d}$; when $N>1, \alpha=1 / 2$ and $d=1$, it is the multiparameter Brownian motion introduced by P. Lévy; see Kahane (1985) and Samorodnitsky and Taqqu (1994) for more historical information, probabilistic and statistical properties of fractional Brownian motion.

By using (1.2) one can verify that $X^{\alpha}$ is self-similar with exponent $\alpha$, i.e. for every constant $c>0$,

$$
\left\{X^{\alpha}(c t), t \in \mathbb{R}^{N}\right\} \stackrel{\mathrm{d}}{=}\left\{c^{\alpha} X^{\alpha}(t), t \in \mathbb{R}^{N}\right\},
$$

where $\stackrel{\mathrm{d}}{=}$ means equality in finite dimensional distributions. Moreover, $X^{\alpha}$ has stationary increments in the strong sense; see Section 8.1 of Samorodnitsky and Taqqu (1994). In particular, $X$ is isotropic in the sense that the distribution of $X(s)-X(t)$ depends only on the Euclidean distance $|s-t|$. Fractional Brownian motion is naturally related to long range dependence which makes it important for modelling phenomena with self-similarity and/or long memory properties. In the last decade the 
literature on statistical analysis and applications of fractional Brownian motion has grown rapidly [cf. Doukhan et al. (2003)].

On the other hand, many data sets from various areas such as image processing, hydrology, geostatistics and spatial statistics have anisotropic nature in the sense that they have different geometric and probabilistic characteristics along different directions, hence fractional Brownian motion is not adequate for modelling such phenomena. Many people have proposed to apply anisotropic Gaussian random fields as more realistic models. See, for example, Davies and Hall (1999), Bonami and Estrade (2003) and Benson, et al. (2006).

Several classes of anisotropic Gaussian random fields have been introduced for theoretical and application purposes. For example, Kamont (1996) introduced fractional Brownian sheets [see the definition in Section 2.1] and studied some of their regularity properties. Benassi et al. (1997) and Bonami and Estrade (2003) considered some anisotropic Gaussian random fields with stationary increments. Biermé et al. (2007) constructed a large class of operator self-similar Gaussian or stable random fields with stationary increments. Anisotropic Gaussian random fields also arise naturally in stochastic partial differential equations [see, e.g., Dalang (1999), Mueller and Tribe (2002), Øksendal and Zhang (2000), Nualart (2006)]; and in studying the most visited sites of symmetric Markov processes [Eisenbaum and Khoshnevisan (2002)]. Hence it is of importance in both theory and applications to investigate the probabilistic and statistical properties of anisotropic random fields.

This paper is concerned with sample path properties of anisotropic Gaussian random fields in general. From the recent works on fractional Brownian sheets [see Section 2.1 for a list of references] it is known that the behavior of anisotropic Gaussian random fields may differ significantly from those of the Brownian sheet and fractional Brownian motion. Our objective is to gather and develop some general methods for studying the analytic and geometric properties of anisotropic Gaussian fields. In particular our results are applicable to the solutions of stochastic partial differential equations including the stochastic heat and wave equations. In a similar vein, Pitt and Robeva (2003), Robeva and Pitt (2004), Balan and Kim (2006) have proposed to study the Markov properties of (anisotropic) Gaussian random fields and the solutions to the stochastic heat equations.

The rest of this paper is organized as follows. Section 2 contains definitions and basic properties of several classes of anisotropic Gaussian random fields including fractional Brownian sheets, Gaussian random fields with stationary increments and solutions to stochastic partial differential equations. We also provide the general conditions [i.e., Conditions $(\mathrm{C} 1),(\mathrm{C} 2),(\mathrm{C} 3)$ and $\left(\mathrm{C} 3^{\prime}\right)$ ] for the Gaussian random fields that will be studied in this paper.

An important technical tool in this paper is the properties of strong local nondeterminism for anisotropic Gaussian random fields, extending the concept of local nondeterminism first introduced by Berman (1973) for Gaussian processes. In Section 3, we recall the recent result of Wu and Xiao (2007) on the property of sectorial local nondeterminism for fractional Brownian sheets; and we prove a sufficient condition for an anisotropic Gaussian field with stationary increments to be strongly locally nondeterministic (with respect to an appropriate metric).

Section 4 is concerned with analytic and asymptotic properties of the sample functions of anisotropic Gaussian fields. We summarize three methods for deriving a sharp modulus of continuity for all anisotropic Gaussian random fields satisfying Condition (C1). The first method is to use an extension of the powerful Garsia-Rodemich-Rumsey continuity lemma [see Arnold and Imkeller (1996), Funaki, Kikuchi and Potthoff (2006), Dalang, Khoshnevisan and Nualart (2007a)]; the second is the "minorizing metric" method of Kwapień and Risiński (2004); and the third is based 
on the Gaussian isoperimetric inequality. While the first two methods have wider applicability, the third method produces more precise results for Gaussian random fields.

Section 5 provides an application of strong local nondeterminism in studying small ball probabilities of anisotropic Gaussian fields.

In Section 6, we consider the Hausdorff and packing dimensions of the range $X\left([0,1]^{N}\right)=$ $\left\{X(t), t \in[0,1]^{N}\right\}$ and graph $\operatorname{Gr} X\left([0,1]^{N}\right)=\left\{(t, X(t)), t \in[0,1]^{N}\right\}$ of $X$. Due to anisotropy, these results are different from the corresponding results for fractional Brownian motion and the Brownian sheet. We also establish an explicit formula for the Hausdorff dimension of the image $X(E)$ in terms of the generalized Hausdorff dimension of $E$ (with respect to an appropriate metric) and the Hurst index $H$. Moreover, when $H=\langle\alpha\rangle$ [see below for the notation], we prove the following uniform Hausdorff dimension result for the images of $X$ : If $N \leq \alpha d$, then with probability one,

$$
\operatorname{dim}_{\mathcal{H}} X(E)=\frac{1}{\alpha} \operatorname{dim}_{\mathcal{H}} E \quad \text { for all Borel sets } E \subseteq(0, \infty)^{N}
$$

This extends the previous results of Monrad and Pitt (1987), Mountford (1989) and Khoshnevisan, $\mathrm{Wu}$ and Xiao (2006) for fractional Brownian motion and the Brownian sheet, respectively, and is another application of the strong local nondeterminism.

In Section 7, we determine the Hausdorff and packing dimensions of the level sets, and establish estimates on the hitting probabilities of Gaussian random fields $X$ satisfying Conditions (C1) and $(\mathrm{C} 2)$.

In Section 8, we study the existence and joint continuity of local times of anisotropic Gaussian random fields under Conditions (C3) and (C3 $\left.3^{\prime}\right)$. Moreover, we discuss local and uniform Hölder conditions of the local times in the set variable and show their applications in evaluating the exact Hausdorff measure of the level sets of $X$.

We end the Introduction with some notation. Throughout this paper, the underlying parameter space is $\mathbb{R}^{N}$ or $\mathbb{R}_{+}^{N}=[0, \infty)^{N}$. We use $|\cdot|$ to denote the Euclidean norm in $\mathbb{R}^{N}$. The inner product and Lebesgue measure in $\mathbb{R}^{N}$ are denoted by $\langle\cdot, \cdot\rangle$ and $\lambda_{N}$, respectively. A typical parameter, $t \in \mathbb{R}^{N}$ is written as $t=\left(t_{1}, \ldots, t_{N}\right)$, or as $\langle c\rangle$ if $t_{1}=\cdots=t_{N}=c$. For any $s, t \in \mathbb{R}^{N}$ such that $s_{j}<t_{j}(j=1, \ldots, N),[s, t]=\prod_{j=1}^{N}\left[s_{j}, t_{j}\right]$ is called a closed interval (or a rectangle). We will let $\mathcal{A}$ denote the class of all closed intervals in $\mathbb{R}^{N}$. For two functions $f$ and $g$, the notation $f(t) \asymp g(t)$ for $t \in T$ means that the function $f(t) / g(t)$ is bounded from below and above by positive constants that do not depend on $t \in T$.

We will use $c$ to denote an unspecified positive and finite constant which may not be the same in each occurrence. More specific constants in Section $i$ are numbered as $c_{i, 1}, c_{i, 2}, \ldots$.

Acknowledgments. This paper is based on lectures given at the Minicourse on Stochastic Partial Differential Equations held at the University of Utah during May 8-19, 2006. The author thanks the organizers, Davar Khoshnevisan and Firas Rassoul-Agha, for their invitation and encouragement. He is also indebted to the participants of the Minicourse for stimulating conversations, to Hermine Bierimé, Erkan Nane, Mark M. Meerschaert, Wensheng Wang, Dongsheng Wu for their comments on the manuscript which have led to many improvements of this paper. 


\section{Examples and general assumptions}

In this section, we give some important examples of anisotropic Gaussian random fields, among them, fractional Brownian sheets are the most studied. We will show that the methods for studying fractional Brownian sheets can be modified to investigate sample path properties of anisotropic Gaussian random fields in general. In $\S 2.4$, we provide the general conditions for the Gaussian random fields that will be studied in this paper.

Even though anisotropic random fields generally do not satisfy the ordinary self-similarity (1.3), they may have certain operator-scaling properties. Following the terminology of Biermé et al. (2007), we say that a random field $X=\left\{X(t), t \in \mathbb{R}^{N}\right\}$ is operator-self-similar [or operatorscaling] in the time variable if there exist a linear operator $A$ on $\mathbb{R}^{N}$ with positive real parts of the eigenvalues and some constant $\beta>0$ such that

$$
\left\{X\left(c^{A} t\right), t \in \mathbb{R}^{N}\right\} \stackrel{d}{=}\left\{c^{\beta} X(t), t \in \mathbb{R}^{N}\right\}, \quad \forall c>0
$$

In the above, $c^{A}$ is the linear operator defined by $c^{A}=\sum_{n=0}^{\infty} \frac{(\ln c)^{n} A^{n}}{n !}$. The linear operator $A$ is called a self-similarity exponent [which may not be unique].

There is also a notion of operator-self-similarity in the space variable [cf. e.g., Mason and Xiao (2001), Xiao (1995)], which will not be discussed in this paper.

\subsection{Fractional Brownian sheets}

Fractional Brownian sheets were first introduced by Kamont (1996) who also studied some of their regularity properties. For a given vector $H=\left(H_{1}, \ldots, H_{N}\right) \in(0,1)^{N}$, an $(N, 1)$-fractional Brownian sheet $B_{0}^{H}=\left\{B_{0}^{H}(t), t \in \mathbb{R}^{N}\right\}$ with Hurst index $H$ is a real-valued, centered Gaussian random field with covariance function given by

$$
\mathbb{E}\left[B_{0}^{H}(s) B_{0}^{H}(t)\right]=\prod_{j=1}^{N} \frac{1}{2}\left(\left|s_{j}\right|^{2 H_{j}}+\left|t_{j}\right|^{2 H_{j}}-\left|s_{j}-t_{j}\right|^{2 H_{j}}\right), \quad s, t \in \mathbb{R}^{N} .
$$

It follows from (2.2) that $B_{0}^{H}(t)=0$ a.s. for every $t \in \mathbb{R}^{N}$ with at least one zero coordinate.

Note that if $N=1$, then $B_{0}^{H}$ is a fractional Brownian motion in $\mathbb{R}$ with Hurst index $H_{1} \in(0,1)$; if $N>1$ and $H=\langle 1 / 2\rangle$, then $B^{H}$ is the Brownian sheet in $\mathbb{R}$. Hence $B_{0}^{H}$ can be regarded as a natural generalization of one parameter fractional Brownian motion as well as a generalization of the Brownian sheet.

It follows from $(2.2)$ that $B_{0}^{H}$ has the following operator-scaling property: For all constants $c>0$,

$$
\left\{B_{0}^{H}\left(c^{A} t\right), t \in \mathbb{R}^{N}\right\} \stackrel{d}{=}\left\{c^{N} B_{0}^{H}(t), t \in \mathbb{R}^{N}\right\}
$$

where $A=\left(a_{i j}\right)$ is the $N \times N$ diagonal matrix with $a_{i i}=1 / H_{i}$ for all $1 \leq i \leq N$ and $a_{i j}=0$ if $i \neq j$. Thus, $B_{0}^{H}$ is operator-self-similar with exponent $A$ and $\beta=N$.

The covariance structure of $B_{0}^{H}$ is more complicated than those of fractional Brownian motion and the Brownian sheet. The following stochastic integral representations are useful. They were established by Ayache et al. (2002) and Herbin (2006), respectively, and can be verified by checking the covariance functions. 
- Moving average representation:

$$
B_{0}^{H}(t)=\kappa_{H}^{-1} \int_{-\infty}^{t_{1}} \cdots \int_{-\infty}^{t_{N}} g(t, s) W(d s)
$$

where $W=\left\{W(s), s \in \mathbb{R}^{N}\right\}$ is a standard real-valued Brownian sheet and

$$
g(t, s)=\prod_{j=1}^{N}\left[\left(\left(t_{j}-s_{j}\right)_{+}\right)^{H_{j}-1 / 2}-\left(\left(-s_{j}\right)_{+}\right)^{H_{j}-1 / 2}\right]
$$

with $s_{+}=\max \{s, 0\}$, and where $\kappa_{H}>0$ is a normalization constant.

To give a harmonizable representation for $B_{0}^{H}$, let us recall briefly the definition of a complexvalued Gaussian measure. Let $(E, \mathcal{E}, \Delta)$ be a measure space and let $\mathcal{A}=\{A \in \mathcal{E}: \Delta(A)<\infty\}$. We say that $\widetilde{\mathcal{M}}$ is a centered complex-valued Gaussian measure on $(E, \mathcal{E}, \Delta)$ if $\{\widetilde{\mathcal{M}}(A), A \in \mathcal{A}\}$ is a centered complex-valued Gaussian process satisfying

$$
\mathbb{E}(\widetilde{\mathcal{M}}(A) \overline{\widetilde{\mathcal{M}}(B)})=\Delta(A \cap B) \quad \text { and } \quad \widetilde{\mathcal{M}}(-A)=\overline{\widetilde{\mathcal{M}}}(A)
$$

for all $A, B \in \mathcal{A}$. The measure $\Delta$ is called the control measure of $\widetilde{\mathcal{M}}$. For any complex valued function $\widetilde{f} \in L^{2}(E, \mathcal{E}, \Delta)$, the stochastic integral $\int_{E} \widetilde{f}(\xi) \widetilde{\mathcal{M}}(d \xi)$ can be defined; see, e.g., Section 7.2.2 of Samorodnitsky and Taqqu (1994). With this notion, we give the following

\section{- Harmonizable representation:}

$$
B_{0}^{H}(t)=K_{H}^{-1} \int_{\mathbb{R}^{N}} \psi_{t}(\lambda) \widetilde{W}(d \lambda),
$$

where $\widetilde{W}$ is a centered complex-valued Gaussian random measure in $\mathbb{R}^{N}$ with Lebesgue control measure and

$$
\psi_{t}(\lambda)=\prod_{j=1}^{N} \frac{e^{i t_{j} \lambda_{j}}-1}{\left|\lambda_{j}\right|^{H_{j}+\frac{1}{2}}},
$$

where $K_{H}>0$ is a constant. Recently, Wang (2007) gave another stochastic integral representation for $B_{0}^{H}$.

Let $B_{1}^{H}, \ldots, B_{d}^{H}$ be $d$ independent copies of $B_{0}^{H}$. Then the $(N, d)$-fractional Brownian sheet with Hurst index $H=\left(H_{1}, \ldots, H_{N}\right)$ is the Gaussian random field $B^{H}=\left\{B^{H}(t): t \in \mathbb{R}^{N}\right\}$ with values in $\mathbb{R}^{d}$ defined by

$$
B^{H}(t)=\left(B_{1}^{H}(t), \ldots, B_{d}^{H}(t)\right), \quad t \in \mathbb{R}^{N} .
$$

Several authors have studied various properties of fractional Brownian sheets. For example, Ayache et al. (2002) provided the moving average representation (2.4) for $B_{0}^{H}$ and studied its sample path continuity as well as its continuity in $H$. Dunker (2000), Mason and Shi (2001), Belinski and Linde (2002), Kühn and Linde (2002) studied the small ball probabilities of $B_{0}^{H}$. Mason and Shi (2001) also computed the Hausdorff dimension of some exceptional sets related to the oscillation of the sample paths of $B_{0}^{H}$. Ayache and Taqqu (2003) derived an optimal wavelet series expansion for fractional Brownian sheet $B_{0}^{H}$; see also Kühn and Linde (2002), Dzhaparidze and van Zanten (2005) for other optimal series expansions for $B_{0}^{H}$. Stochastic partial differential 
equations driven by fractional Brownian sheets have been studied by Øksendal and Zhang (2000), and by $\mathrm{Hu}, \varnothing \mathrm{ksendal}$ and Zhang (2000).

For fractal properties, Kamont (1996) and Ayache (2004) studied the box and Hausdorff dimensions of the graph set of an $(N, 1)$-fractional Brownian sheet. Ayache and Xiao (2005) investigated the uniform and local asymptotic properties of $B^{H}$ by using wavelet methods, and determined the Hausdorff dimensions of the image $B^{H}\left([0,1]^{N}\right)$, the graph $\operatorname{Gr} B^{H}\left([0,1]^{N}\right)$ and the level set $L_{x}=\left\{t \in(0, \infty)^{N}: B^{H}(t)=x\right\}$, where $x \in \mathbb{R}^{d}$. Further results on the geometric and Fourier analytic properties of the images of $B^{H}$ can be found in Wu and Xiao (2007).

Xiao and Zhang (2002) studied the existence of local times of an $(N, d)$-fractional Brownian sheet $B^{H}$ and proved a sufficient condition for the joint continuity of the local times. Ayache, Wu and Xiao (2006) established the joint continuity of the local times under the optimal condition and studied the local and uniform Hölder conditions for the maximum local times. Related to the above results, we mention that Tudor and Xiao (2007) have obtained results on Hausdorff dimensions of the sample paths, local times and their chaos expansion for $(N, d)$-bifractional Brownian sheets.

\subsection{Anisotropic Gaussian random fields with stationary increments}

Let $X=\left\{X(t), t \in \mathbb{R}^{N}\right\}$ be a real-valued, centered Gaussian random field with $X(0)=0$. We assume that $X$ has stationary increments and continuous covariance function $R(s, t)=\mathbb{E}[X(s) X(t)]$. According to Yaglom (1957), $R(s, t)$ can be represented as

$$
R(s, t)=\int_{\mathbb{R}^{N}}\left(e^{i\langle s, \xi\rangle}-1\right)\left(e^{-i\langle t, \xi\rangle}-1\right) \Delta(d \xi)+\langle s, \Theta t\rangle,
$$

where $\langle x, y\rangle$ is the ordinary inner product in $\mathbb{R}^{N}, \Theta$ is an $N \times N$ non-negative definite matrix and $\Delta(d \xi)$ is a nonnegative symmetric measure on $\mathbb{R}^{N} \backslash\{0\}$ satisfying

$$
\int_{\mathbb{R}^{N}} \frac{|\xi|^{2}}{1+|\xi|^{2}} \Delta(d \xi)<\infty
$$

The measure $\Delta$ and its density (if it exists) $f(\xi)$ are called the spectral measure and spectral density of $X$, respectively.

It follows from (2.7) that $X$ has the following stochastic integral representation:

$$
X(t)=\int_{\mathbb{R}^{N}}\left(e^{i\langle t, \xi\rangle}-1\right) \widetilde{\mathcal{M}}(d \xi)+\langle\mathbf{Y}, t\rangle,
$$

where $\mathbf{Y}$ is an $N$-dimensional Gaussian random vector with mean 0 and covariance matrix $\Theta$, and where $\widetilde{\mathcal{M}}$ is a centered complex-valued Gaussian random measure in $\mathbb{R}^{N}$ with control measure $\Delta$, which is independent of $\mathbf{Y}$. Since the linear term $\langle\mathbf{Y}, t\rangle$ in (2.9) will not have any effect on the problems considered in this paper, we will from now on assume $\mathbf{Y}=0$. This is equivalent to assuming $\Theta=0$ in (2.7). Consequently, we have

$$
\sigma^{2}(h)=\mathbb{E}\left[(X(t+h)-X(t))^{2}\right]=2 \int_{\mathbb{R}^{N}}(1-\cos \langle h, \xi\rangle) \Delta(d \xi) .
$$

It is important to note that $\sigma^{2}(h)$ is a negative definite function [see Berg and Forst (1975)] and, by the Lévy-Khintchine formula, can be viewed as the characteristic exponent of a symmetric infinitely divisible distribution. 
If the function $\sigma^{2}(h)$ depends only on $|h|$, then $X$ is called an isotropic random field. We say that a Gaussian random field $X$ is approximately isotropic if $\sigma^{2}(h) \asymp \phi(|h|)$ in a neighborhood of $h=0$ for some nonnegative function $\phi$. Sample path properties of such Gaussian random fields have been studied widely. See Xiao (1997b, 2007), Shieh and Xiao (2006) and the references therein for more information. The results in Ayache and Xiao (2005), Wu and Xiao (2007) on fractional Brownian sheets indicate that the properties of anisotropic Gaussian random fields can be very different and often more difficult to be established.

Many Gaussian random fields can be constructed by choosing the spectral measures appropriately. For example, if we consider the spectral density

$$
f(\xi)=\frac{1}{\left(\sum_{j=1}^{N}\left|\xi_{j}\right|^{H_{j}}\right)^{2+Q}}, \quad \forall \xi \in \mathbb{R}^{N} \backslash\{0\},
$$

where the constants $H_{j} \in(0,1)$ for $j=1, \ldots, N$ and $Q=\sum_{j=1}^{N} H_{j}^{-1}$, then the corresponding Gaussian random field $X$ has stationary increments and is operator-self-similar with exponent $A=\left(a_{i j}\right)$, where $a_{i i}=H_{i}^{-1}$ and $a_{i j}=0$ if $i \neq j$ and $\beta=1$. This Gaussian random field is similar to that in Example 3 of Bonami and Estrade (2003).

The following class of Gaussian random fields constructed by Biermé, Meerschaert and Scheffler (2007, Section 4) is more general.

Theorem 2.1 Let $A$ be a real $N \times N$ matrix with the real parts of the eigenvalues $1<a_{1} \leq a_{2} \leq$ $\cdots \leq a_{N}$ and let $Q=\operatorname{trace}(A)$. If $\psi: \mathbb{R}^{N} \rightarrow[0, \infty)$ is a continuous, $A^{\prime}$-homogeneous function [ i.e., $\psi\left(c^{A^{\prime}} \xi\right)=c \psi(\xi)$ for all $c>0$ and $\xi \in \mathbb{R}^{N}$. Here $A^{\prime}$ denotes the transpose of $\left.A\right]$ such that $\psi(\xi)>0$ for $\xi \neq 0$. Then the Gaussian random field

$$
X_{\psi}(t)=\operatorname{Re} \int_{\mathbb{R}^{N}}\left(e^{i\langle t, \xi\rangle}-1\right) \frac{\widetilde{\mathcal{W}}(d \xi)}{\psi(\xi)^{1+Q / 2}}, \quad x \in \mathbb{R}^{N},
$$

where $\widetilde{\mathcal{W}}$ is a centered complex-valued Gaussian random measure in $\mathbb{R}^{N}$ with Lebesgue control measure, has stationary increments and is operator-self-similar in the sense of (2.1) with exponent $A$ and $\beta=1$.

Compared with (2.9), we see that the spectral measure of $X_{\psi}$ is $\Delta(d \xi)=\psi(\xi)^{-(2+Q)} d \xi$. As the results of this paper will suggest, the sample functions of $X_{\psi}$ share many properties with fractional Brownian sheets and many of them can be described in terms of the real parts of the eigenvalues of $A$. See Biermé, Estrade, Meerschaert and Xiao (2008) for more details.

\subsection{Solutions to stochastic partial differential equations}

Gaussian random fields arise naturally as solutions to stochastic partial differential equations. In the following we list as examples the solutions to the stochastic heat equation and stochastic wave equation, and discuss possible ways to study their sample path properties using general methods for Gaussian random fields. We refer to Walsh (1986), Dalang (1999), Dalang and Frangos (1998), Dalang and Mueller (2003), Mueller and Tribe (2002), Dalang and Sanz-Solé (2005, 2007), Dalang, Khoshnevisan and Nualart (2007a, 2007b) and the articles in this volume for more information.

\subsubsection{The stochastic heat equation}


Funaki's model for random string in $\mathbb{R}$ is specified by the following stochastic heat equation:

$$
\frac{\partial u(t, x)}{\partial t}=\frac{\partial^{2} u(t, x)}{\partial x^{2}}+\dot{W}
$$

where $\dot{W}(x, t)$ is an $\mathbb{R}$-valued space-time white noise, which is assumed to be adapted with respect to a filtered probability space $\left(\Omega, \mathcal{F}, \mathcal{F}_{t}, \mathbb{P}\right)$, where $\mathcal{F}$ is complete and the filtration $\left\{\mathcal{F}_{t}, t \geq 0\right\}$ is right continuous; see Funaki (1983) and Mueller and Tribe (2002) for more information.

Recall from Mueller and Tribe (2002) that a solution of (2.13) is defined as an $\mathcal{F}_{t}$-adapted, continuous random field $\left\{u(t, x), t \in \mathbb{R}_{+}, x \in \mathbb{R}\right\}$ with values in $\mathbb{R}$ satisfying the following properties:

(i) $u(0, \cdot) \in \mathcal{E}_{\exp }$ almost surely and is adapted to $\mathcal{F}_{0}$, where $\mathcal{E}_{\exp }=\cup_{\lambda>0} \mathcal{E}_{\lambda}$ and

$$
\mathcal{E}_{\lambda}=\left\{f \in C(\mathbb{R}):|f(x)| e^{-\lambda|x|} \rightarrow 0 \text { as }|x| \rightarrow \infty\right\} ;
$$

(ii) For every $t>0$, there exists $\lambda>0$ such that $u(s, \cdot) \in \mathcal{E}_{\lambda}$ for all $s \leq t$, almost surely;

(iii) For every $t>0$ and $x \in \mathbb{R}$, the following Green's function representation holds

$$
u(t, x)=\int_{\mathbb{R}} G(t, x-y) u(0, y) d y+\int_{0}^{t} \int_{\mathbb{R}} G(t-r, x-y) W(d y d r)
$$

where $G(t, x)=\frac{1}{\sqrt{4 \pi t}} e^{-\frac{x^{2}}{4 t}}$ is the fundamental solution of the heat equation.

We call each solution $\left\{u(t, x), t \in \mathbb{R}_{+}, x \in \mathbb{R}\right\}$ of (2.13) a random string process with values in $\mathbb{R}$, or simply a random string as in Mueller and Tribe (2002). Note that, in general, a random string may not be Gaussian, a powerful step in the proofs of Mueller and Tribe (2002) is to reduce the problems about a general random string process to those of the stationary pinned string $U_{0}=\left\{U_{0}(t, x), t \in \mathbb{R}_{+}, x \in \mathbb{R}\right\}$, obtained by taking the initial function $u(0, \cdot)$ in $(2.14)$ to be defined by

$$
u(0, x)=\int_{0}^{\infty} \int_{\mathbb{R}}(G(r, x-z)-G(r, z)) \widehat{W}(d z d r),
$$

where $\widehat{W}$ is a space-time white noise independent of the white noise $\dot{W}$. Consequently, the stationary pinned string is a continuous version of the following Gaussian field

$$
\begin{aligned}
U_{0}(t, x)=\int_{0}^{\infty} \int_{\mathbb{R}} & (G(t+r, x-z)-G(t+r, z)) \widehat{W}(d z d r) \\
& +\int_{0}^{t} \int_{\mathbb{R}} G(r, x-z) W(d z d r),
\end{aligned}
$$

Mueller and Tribe (2002) proved that the Gaussian field $U_{0}=\left\{U_{0}(t, x), t \in \mathbb{R}_{+}, x \in \mathbb{R}\right\}$ has stationary increments and satisfies the Conditions $(\mathrm{C} 1)$ and $(\mathrm{C} 2)$ in Section 2.4. Let $U_{1}, \ldots, U_{d}$ be $d$ independent copies of $U_{0}$, and consider the Gaussian random field $U=\left\{U(t, x), t \in \mathbb{R}_{+}, x \in \mathbb{R}\right\}$ with values in $\mathbb{R}^{d}$ defined by $U(t, x)=\left(U_{1}(t, x), \ldots, U_{d}(t, x)\right)$. Mueller and Tribe (2002) found necessary and sufficient conditions [in terms of the dimension $d$ ] for $U$ to hit points or to have double points of various types. They also studied the question of recurrence and transience for $\left\{U(t, x), t \in \mathbb{R}_{+}, x \in \mathbb{R}\right\}$. Continuing the work of Mueller and Tribe (2002), Wu and Xiao (2006) 
studied the fractal properties of various random sets generated by the random string processes. Further results on hitting probabilities of non-linear stochastic heat equations can be found in Dalang et al. (2006, 2007).

On the other hand, Robeva and Pitt (2004, Proposition 3) showed that the Gaussian random field

$$
u_{0}(t, x)=\frac{1}{2 \pi} \int_{\mathbb{R}^{2}} \frac{e^{i\left(\xi_{1} t+\xi_{2} x\right)}-1}{i \xi_{1}+\xi_{2}^{2}} \widetilde{\mathcal{W}}\left(d \xi_{1}, d \xi_{2}\right), \quad \forall t \in \mathbb{R}_{+}, x \in \mathbb{R}
$$

is another solution to $(2.13)$ satisfying $u_{0}(0,0)=0$. Here $\widetilde{\mathcal{W}}$ is a centered complex Gaussian random measure in $\mathbb{R}^{2}$ with Lebesgue control measure. This Gaussian random field has stationary increments with spectral density

$$
f(\xi)=\frac{1}{\xi_{1}^{2}+\xi_{2}^{4}} .
$$

This density function is comparable to (2.11) with $H_{1}=1 / 4, H_{2}=1 / 2$ and $Q=6$. Hence, it follows from Theorem 3.2 that the Gaussian field $\left\{u_{0}(t, x), t \in \mathbb{R}_{+}, x \in \mathbb{R}\right\}$ satisfies the Conditions $(\mathrm{C} 1)$ and $\left(\mathrm{C} 3^{\prime}\right)$ in $\S 2.4$. If we define a $(2, d)$-Gaussian random field $\left\{u(t, x), t \in \mathbb{R}_{+}, x \in \mathbb{R}\right\}$ by $u(t, x)=\left(u_{1}(t, x), \ldots, u_{d}(t, x)\right)$, where $u_{1}, \ldots, u_{d}$ are independent copies of $u_{0}$, then many of its sample path properties follow from the results in later sections of this paper.

If $x \in \mathbb{R}^{N}$ and $N \geq 2$, the stochastic heat equation (2.13) has no process solution the solution is a random Schwartz distribution]. It might be helpful to remark that our random field notation is different from that in the references on s.p.d.e.'s: now the parameter $(t, x) \in \mathbb{R}^{N+1}$ and $\mathbb{R}^{d}$ is reserved for the state space of random fields.

The approach of Dalang (1999) is to replace $\dot{W}$ by a Gaussian noise $\dot{F}$ which is white in time and has spatial covariance induced by a kernel $f$, which is the Fourier transform of a tempered measure $\mu$ in $\mathbb{R}^{N}$. The covariance of $F$ is of the form

$$
\mathbb{E}(F(d t, d x) F(d s, d y))=\delta(t-s) f(x-y),
$$

where $\delta(\cdot)$ is the Dirac delta function. The case $f(r)=\delta(r)$ would correspond to the case of spacetime white noise. More precisely, let $\mathcal{D}\left(\mathbb{R}^{N+1}\right)$ be the topological space of functions $\phi \in C_{0}^{\infty}\left(\mathbb{R}^{N+1}\right)$ with the topology that corresponds to the following notion of convergence: $\phi_{n} \rightarrow \phi$ if and only if the following two conditions hold:

(i). There exists a compact set $K \subseteq \mathbb{R}^{N+1}$ such that $\operatorname{supp}\left(\phi_{n}-\phi\right) \subseteq K$ for all $n \geq 1$, and

(ii). $\lim _{n \rightarrow \infty} D^{a} \phi_{n}=D^{a} \phi$ uniformly on $K$ for every multi-index $a$.

Let $F=\left\{F(\phi), \phi \in \mathcal{D}\left(\mathbb{R}^{N+1}\right)\right\}$ be an $L^{2}(\Omega, \mathcal{F}, \mathbb{P})$-valued, centered Gaussian process with covariance of the form $(\phi, \psi) \mapsto \mathbb{E}(F(\phi) F(\psi))=J(\phi, \psi)$, where

$$
J(\phi, \psi)=\int_{\mathbb{R}_{+}} d t \int_{\mathbb{R}^{N}} d x \int_{\mathbb{R}^{N}} \phi(t, x) f(x-y) \psi(t, y) d y .
$$

As shown by Dalang (1999), $\phi \mapsto F(\phi)$ can be extended to a worthy martingale measure $(t, A) \mapsto M_{t}(A)$ [in the sense of Walsh (1986, pages 289-290)] with covariance measure

$$
Q([0, t] \times A \times B)=\langle M(A) ; M(B)\rangle_{t}=t \int_{\mathbb{R}^{N}} d x \int_{\mathbb{R}^{N}} \mathbf{1}_{A}(x) f(x-y) \mathbf{1}_{B}(y) d y
$$


and dominating measure $K \equiv Q$ such that

$$
F(\phi)=\int_{\mathbb{R}_{+}} \int_{\mathbb{R}^{N}} \phi(t, x) M(d t, d x), \quad \forall \phi \in \mathcal{D}\left(\mathbb{R}^{N+1}\right) .
$$

Moreover, Dalang (1999) constructed generalized stochastic integrals with respect to the martingale measure $M$.

Now we consider the stochastic heat equation with vanishing initial conditions, written formally as

$$
\frac{\partial u(t, x)}{\partial t}=\Delta u(t, x)+\dot{F}, \quad \forall(t, x) \in(0, T) \times \mathbb{R}^{N}
$$

and $u(0, \cdot) \equiv 0$. Here $T>0$ is any fixed constant and $\dot{F}$ is the Gaussian noise defined above.

Dalang (1999) proved that (2.19) has a process solution if and only if

$$
\int_{\mathbb{R}^{N}} \frac{1}{1+|\xi|^{2}} \mu(d \xi)<\infty .
$$

Under this condition, the mean zero Gaussian field $u=\left\{u(t, x) ; t \in[0, T], x \in \mathbb{R}^{N}\right\}$ defined by

$$
u(t, x)=\int_{0}^{T} \int_{\mathbb{R}^{N}} G(t-s, x-y) M(d s, d y)
$$

is the process solution of the stochastic heat equation (2.19) with vanishing initial condition. In the above, $G(r, x)=(4 \pi r)^{-N / 2} \exp \left(-|x|^{2} /(4 r)\right)\left(r>0, x \in \mathbb{R}^{N}\right)$ is the fundamental solution of the heat equation.

Many interesting examples can be constructed by choosing $\mu(d \xi)$ suitably; see Dalang (1999) and Balan and Kim (2006). As we mentioned in the Introduction, Robeva and Pitt (2004), and Balan and Kim (2006) studied the Markov property of the solution of stochastic heat equation (2.19). In view of the results in this paper, it would be interesting to see when the solutions of (2.19) satisfy Conditions (C3) or $\left(\mathrm{C} 3^{\prime}\right)$ in $\S 2.4$.

\subsubsection{The stochastic wave equation}

The stochastic wave equation in one spatial dimension [i.e., $N=1$ ]

$$
\frac{\partial^{2} u(t, x)}{\partial^{2} t}-\frac{\partial^{2} u(t, x)}{\partial x^{2}}=\dot{W}(t, x), \quad t>0, x \in \mathbb{R}
$$

driven by the white noise was considered by Walsh (1986) and many other authors [see Dalang and Frangos (1998) and Dalang and Mueller (2003) for a list of the references]. In spatial dimension two or higher, however, the stochastic wave equation driven by the white noise has no solution in the space of real valued measurable processes [see Walsh (1986)].

For $N=2$, Dalang and Frangos (1998) considered the stochastic wave equation driven by the Gaussian noise $\dot{F}$ with covariance (2.17):

$$
\left\{\begin{array}{l}
\frac{\partial^{2} u(t, x)}{\partial t^{2}}=\Delta u(t, x)+\dot{F}, \\
u(0, x)=0, \\
\frac{\partial u}{\partial t}(0, x)=0,
\end{array} \quad \forall(t, x) \in(0, \infty) \times \mathbb{R}^{2} .\right.
$$


They proved that (2.23) has a process solution $u=\left\{u(t, x): t \geq 0, x \in \mathbb{R}^{2}\right\}$ if and only if

$$
\int_{0+} f(r) r \log \left(\frac{1}{r}\right) d r<\infty
$$

where $f$ is the kernel in (2.17). Under the latter condition, $u=\left\{u(t, x): t \geq 0, x \in \mathbb{R}^{2}\right\}$ can be represented as

$$
u(t, x)=\int_{0}^{t} \int_{\mathbb{R}^{2}} S(t-s, x-y) M(d s, d y),
$$

where $S(t, x)=\frac{1}{2 \pi}\left(t^{2}-|x|^{2}\right)^{-1 / 2} \mathbb{1}_{\{|x|<t\}}$. Sample path regularity of the solution $\{u(t, x): t \geq 0, x \in$ $\left.\mathbb{R}^{2}\right\}$ has been investigated by Dalang and Frangos (1998), and Dalang and Sanz-Solé (2005).

For the stochastic wave equation with spatial dimension three, we refer to Dalang and Mueller (2003), and Dalang and Sanz-Solé (2007) for information on the existence of a process solution and its sample path regularities. It seems that, in all the cases considered so far, the questions on fractal properties, existence and regularity of the local times of the solutions remain to be investigated.

\subsection{General assumptions}

Let $X=\left\{X(t), t \in \mathbb{R}^{N}\right\}$ be a Gaussian random field in $\mathbb{R}^{d}$ defined on some probability space $(\Omega, \mathcal{F}, \mathbb{P})$ by

$$
X(t)=\left(X_{1}(t), \ldots, X_{d}(t)\right), \quad t \in \mathbb{R}^{N},
$$

where $X_{1}, \ldots, X_{d}$ are independent copies of $X_{0}$. We assume that $X_{0}$ is a mean zero Gaussian random field with $X_{0}(0)=0$ a.s.

Let $\left(H_{1}, \ldots, H_{N}\right) \in(0,1)^{N}$ be a fixed vector. In order to study anisotropic Gaussian fields, we have found the following metric $\rho$ on $\mathbb{R}^{N}$ is often more convenient than the Euclidean metric:

$$
\rho(s, t)=\sum_{j=1}^{N}\left|s_{j}-t_{j}\right|^{H_{j}}, \quad \forall s, t \in \mathbb{R}^{N} .
$$

For any $r>0$ and $t \in \mathbb{R}^{N}$, we denote by $B_{\rho}(t, r)=\left\{s \in \mathbb{R}^{N}: \rho(s, t) \leq r\right\}$ the closed (or open) ball in the metric $\rho$.

Let $I \in \mathcal{A}$ be a fixed closed interval, and we will consider various sample path properties of $X(t)$ when $t \in I$. For simplicity we will mostly assume $I=[\varepsilon, 1]^{N}$, where $\varepsilon \in(0,1)$ is fixed. Typically, the assumption for $I$ to be away from the axis is only needed for Gaussian fields similar to fractional Brownian sheets. Even in these later cases, many results, such as those on Hausdorff and packing dimensions, remain to be true for $I=[0,1]^{N}$.

Many sample path properties of $X$ can be determined by the following function:

$$
\sigma^{2}(s, t)=\mathbb{E}\left(X_{0}(s)-X_{0}(t)\right)^{2}, \quad \forall s, t \in \mathbb{R}^{N} .
$$

In this paper, we will make use of the following general conditions on $X_{0}$ :

(C1). There exist positive constants $c_{2,1}, \ldots, c_{2,4}$ such that

$$
c_{2,1} \leq \sigma^{2}(t):=\sigma^{2}(0, t) \leq c_{2,2}, \quad \forall t \in I
$$


and

$$
c_{2,3} \sum_{j=1}^{N}\left|s_{j}-t_{j}\right|^{2 H_{j}} \leq \sigma^{2}(s, t) \leq c_{2,4} \sum_{j=1}^{N}\left|s_{j}-t_{j}\right|^{2 H_{j}}
$$

for all $s, t \in I$. It may be helpful to note that (2.29) is in terms of $\rho(s, t)^{2}$.

(C2). There exists a constant $c_{2,5}>0$ such that for all $s, t \in I$,

$$
\operatorname{Var}\left(X_{0}(t) \mid X_{0}(s)\right) \geq c_{2,5} \rho(s, t)^{2} .
$$

Here and in the sequel, $\operatorname{Var}(Y \mid Z)$ denotes the conditional variance of $Y$ given $Z$.

(C3). There exists a constant $c_{2,6}>0$ such that for all integers $n \geq 1$ and all $u, t^{1}, \ldots, t^{n} \in I$,

$$
\operatorname{Var}\left(X_{0}(u) \mid X_{0}\left(t^{1}\right), \ldots, X_{0}\left(t^{n}\right)\right) \geq c_{2,6} \sum_{j=1}^{N} \min _{0 \leq k \leq n}\left|u_{j}-t_{j}^{k}\right|^{2 H_{j}}
$$

where $t_{j}^{0}=0$ for every $j=1, \ldots, N$.

$\left(\mathrm{C} 3^{\prime}\right)$. There exists a constant $c_{2,7}>0$ such that for all integers $n \geq 1$ and all $u, t^{1}, \ldots, t^{n} \in I$,

$$
\operatorname{Var}\left(X_{0}(u) \mid X_{0}\left(t^{1}\right), \ldots, X_{0}\left(t^{n}\right)\right) \geq c_{2,7} \min _{0 \leq k \leq n} \rho\left(u, t^{k}\right)^{2}
$$

where $t^{0}=0$.

Remark 2.2 The following are some remarks about the above conditions.

- Conditions (C1)-(C3) can be significantly weakened and/or modified in various parts of the paper to obtain more general results. The present formulation of these conditions has the advantage that it is more convenient and produces cleaner results.

- Condition (2.28) assumes that $X$ is non-degenerate on $I$. If (2.29) holds for $s=0$ as well, then $(2.28)$ is automatically true.

- Under condition (C1), $X$ has a version which has continuous sample functions on $I$ almost surely. Henceforth we will assume without loss of generality that the Gaussian random field $X$ has continuous sample paths.

- Conditions (C1) and (C2) are related. It is easy to see that (C1) implies that $\operatorname{Var}\left(X_{0}(t) \mid X_{0}(s)\right)$ $\leq c_{2,4} \sum_{j=1}^{N}\left|s_{j}-t_{j}\right|^{2 H_{j}}$ for all $s, t \in I$ and, on the other hand, (C2) implies $\sigma^{2}(s, t) \geq$ $c_{2,5} \sum_{j=1}^{N}\left|s_{j}-t_{j}\right|^{2 H_{j}}$. Moreover, if the function $\sigma(0, t)$ satisfies certain smoothness condition, say, it has continuous first order derivatives on $I$, then one can show that $(\mathrm{C} 1)$ implies $(\mathrm{C} 2)$ by using the following fact [which can be easily verified]: If $(U, V)$ is a Gaussian vector, then

$$
\operatorname{Var}(U \mid V)=\frac{\left(\rho_{U, V}^{2}-\left(\sigma_{U}-\sigma_{V}\right)^{2}\right)\left(\left(\sigma_{U}+\sigma_{V}\right)^{2}-\rho_{U, V}^{2}\right)}{4 \sigma_{V}^{2}},
$$

where $\rho_{U, V}^{2}=\mathbb{E}\left[(U-V)^{2}\right], \sigma_{U}^{2}=\mathbb{E}\left(U^{2}\right)$ and $\sigma_{V}^{2}=\mathbb{E}\left(V^{2}\right)$. 
- Pitt (1978) proved that fractional Brownian motion $X^{\alpha}$ satisfies Condition $\left(\mathrm{C} 3{ }^{\prime}\right)$ for all $I \in \mathcal{A}$ with $H=\langle\alpha\rangle$; Khoshnevisan and Xiao (2007a) proved that the Brownian sheet satisfies the property (C3) with $H=\langle 1 / 2\rangle$ for all $I \in \mathcal{A}$ which are away from the boundary of $\mathbb{R}_{+}^{N}$. It has been proved in Ayache and Xiao (2005), Wu and Xiao (2007) that, for every $\varepsilon \in(0,1)$, fractional Brownian sheets satisfy Conditions (C1), (C2) and (C3) for all $I \subseteq[\varepsilon, \infty)^{N}$.

- Let $X$ be a Gaussian random field with stationary increments and spectral density comparable to (2.11). Then one can verify that $X$ satisfies Condition (C1). In the next section, we will prove that $X$ satisfies Condition $\left(\mathrm{C}^{\prime}\right)$ [thus it also satisfies (C2)]. Therefore, all the results in this paper are applicable to such Gaussian random fields.

- Note that Condition (C3') implies (C3). It can be verified that the converse does not even hold for the Brownian sheet [this is an exercise]. Roughly speaking, when $H=\langle\alpha\rangle$, the behavior of a Gaussian random field $X$ satisfying conditions $(\mathrm{C} 1)$ and $\left(\mathrm{C} 3^{\prime}\right)$ is comparable to that of a fractional Brownian motion of index $\alpha$; while the behavior of a Gaussian random field $X$ satisfying conditions $(\mathrm{C} 1)$ and $(\mathrm{C} 3)$ [but not $\left(\mathrm{C} 3^{\prime}\right)$ ] is comparable to that of a fractional Brownian sheet. Hence, in analogy to the terminology respectively for fractional Brownian motion and the Brownian sheet, Condition $\left(\mathrm{C} 3^{\prime}\right)$ will be called the strong local nondeterminism [in metric $\rho$ ] and Condition (C3) will be called the sectorial local nondeterminism.

- It is well-known that there is a tight relation between Gaussian processes and operators in Hilbert spaces; see Li and Shao (2001) and the references therein. Recently Linde (2007) has extended the notion of strong local nondeterminism to a linear operator $u: \mathcal{H} \rightarrow C(T)$, where $\mathcal{H}$ is a real Hilbert space and $C(T)$ is the Banach space of continuous functions on the compact metric space $T$, and applied this property to derive a lower bound for the entropy number of $u$. As examples, Linde (2007) shows that the integral operators related to fractional Brownian motion and fractional Brownian sheets are strongly locally nondeterministic in his sense. Following this line, it would be interesting to further study the properties of strong local nondeterminism analogous to $(\mathrm{C} 3)$ and $\left(\mathrm{C} 3^{\prime}\right)$ for linear operators related to anisotropic Gaussian random fields such as the solutions to the stochastic heat and wave equations.

\section{Properties of strong local nondeterminism}

One of the main difficulties in studying sample path properties of anisotropic Gaussian random fields such as fractional Brownian sheets is the complexity of their dependence structure. For example, unlike fractional Brownian motion which is locally nondeterministic [see Pitt (1978)] or the Brownian sheet which has independent increments, a fractional Brownian sheet has neither of these properties. The same is true for anisotropic Gaussian random fields in general. The main technical tool which we will apply to study anisotropic Gaussian random fields is the properties of strong local nondeterminism [SLND] and sectorial local nondeterminism.

Recall that the concept of local nondeterminism was first introduced by Berman (1973) to unify and extend his methods for studying local times of real-valued Gaussian processes, and then extended by Pitt (1978) to Gaussian random fields. The notion of strong local nondeterminism was later developed to investigate the regularity of local times, small ball probabilities and other sample 
path properties of Gaussian processes and Gaussian random fields. We refer to Xiao $(2006,2007)$ for more information on the history and applications of the properties of local nondeterminism.

For Gaussian random fields, the aforementioned properties of local nondeterminism can only be satisfied by those with approximate isotropy. It is well-known that the Brownian sheet does not satisfy the properties of local nondeterminism in the senses of Berman or Pitt. Because of this, many problems for fractional Brownian motion and the Brownian sheet have to be investigated using different methods.

Khoshnevisan and Xiao (2007a) have recently proved that the Brownian sheet satisfies the sectorial local nondeterminism [i.e., (C3) with $H=\langle 1 / 2\rangle$ ] and applied this property to study various analytic and geometric properties of the Brownian sheet; see also Khoshnevisan, Wu and Xiao (2006).

$\mathrm{Wu}$ and Xiao (2007) extended the result of Khoshnevisan and Xiao (2007a) and proved that fractional Brownian sheet $B_{0}^{H}$ satisfies Condition (C3).

Theorem 3.1 Let $B_{0}^{H}=\left\{B_{0}^{H}(t), t \in \mathbb{R}^{N}\right\}$ be an (N,1)-fractional Brownian sheet with index $H=\left(H_{1}, \ldots, H_{N}\right) \in(0,1)^{N}$. For any fixed number $\varepsilon \in(0,1)$, there exists a positive constant $c_{3,1}$, depending on $\varepsilon, H$ and $N$ only, such that for all positive integers $n \geq 1$, and all $u, t^{1}, \ldots, t^{n}$ $\in[\varepsilon, \infty)^{N}$, we have

$$
\operatorname{Var}\left(B_{0}^{H}(u) \mid B_{0}^{H}\left(t^{1}\right), \ldots, B_{0}^{H}\left(t^{n}\right)\right) \geq c_{3,1} \sum_{j=1}^{N} \min _{0 \leq k \leq n}\left|u_{j}-t_{j}^{k}\right|^{2 H_{j}},
$$

where $t_{j}^{0}=0$ for $j=1, \ldots, N$.

Proof While the argument of Khoshnevisan and Xiao (2007a) relies on the property of independent increments of the Brownian sheet and its connection to Brownian motion, the proof for $B_{0}^{H}$ is based on a Fourier analytic argument in Kahane (1985, Chapter 18) and the harmonizable representation (2.5) of $B_{0}^{H}$. We refer to $\mathrm{Wu}$ and Xiao (2007) for details.

Now we prove a sufficient condition for an anisotropic Gaussian random field with stationary increments to satisfy Condition $\left(\mathrm{C} 3^{\prime}\right)$.

Theorem 3.2 Let $X=\left\{X(t), t \in \mathbb{R}^{N}\right\}$ be a centered Gaussian random field in $\mathbb{R}$ with stationary increments and spectral density $f(\lambda)$. Assume that there is a vector $H=\left(H_{1}, \ldots, H_{N}\right) \in(0,1)^{N}$ such that

$$
f(\lambda) \asymp \frac{1}{\left(\sum_{j=1}^{N}\left|\lambda_{j}\right|^{H_{j}}\right)^{2+Q}}, \quad \forall \lambda \in \mathbb{R}^{N} \backslash\{0\},
$$

where $Q=\sum_{j=1}^{N} \frac{1}{H_{j}}$. Then there exists a constant $c_{3,2}>0$ such that for all $n \geq 1$, and all $u, t^{1}, \ldots, t^{n} \in \mathbb{R}^{N}$,

$$
\operatorname{Var}\left(X(u) \mid X\left(t^{1}\right), \ldots, X\left(t^{n}\right)\right) \geq c_{3,2} \min _{0 \leq k \leq n} \rho\left(u, t^{k}\right)^{2}
$$

where $t^{0}=0$. 
Remark 3.3 The following are some comments about Theorem 3.2.

(i) When $H_{1}=\cdots=H_{N},(3.3)$ is of the same form as the SLND of fractional Brownian motion [cf. Pitt (1978)]. As shown by Xiao (1997b, 2007) and Shieh and Xiao (2006), many sample path properties of such Gaussian random fields are similar to those of fractional Brownian motion.

(ii) Condition (3.2) can be significantly weakened. In particular, one can prove that similar results hold for Gaussian random fields with stationary increments and discrete spectrum measures; see Xiao (2008) for details.

(iii) It would be interesting to study under which conditions the solutions to the stochastic heat and wave equations (2.19) and (2.23) are strongly local nondeterministic.

Proof of Theorem 3.2 Denote $r \equiv \min _{0 \leq k \leq n} \rho\left(u, t^{k}\right)$. Since the conditional variance in (3.3) is the square of the $L^{2}(\mathbb{P})$-distance of $X(u)$ from the subspace generated by $\left\{X\left(t^{1}\right), \ldots, X\left(t^{n}\right)\right\}$, it is sufficient to prove that for all $a_{k} \in \mathbb{R}(1 \leq k \leq n)$,

$$
\mathbb{E}\left(X(u)-\sum_{k=1}^{n} a_{k} X\left(t^{k}\right)\right)^{2} \geq c_{3,2} r^{2}
$$

and $c_{3,2}>0$ is a constant which may only depend on $H$ and $N$.

By the stochastic integral representation (2.9) of $X$, the left hand side of (3.4) can be written as

$$
\mathbb{E}\left(X(u)-\sum_{k=1}^{n} a_{k} X\left(t^{k}\right)\right)^{2}=\int_{\mathbb{R}^{N}}\left|e^{i\langle u, \lambda\rangle}-1-\sum_{k=1}^{n} a_{k}\left(e^{i\left\langle t^{k}, \lambda\right\rangle}-1\right)\right|^{2} f(\lambda) d \lambda .
$$

Hence, we only need to show

$$
\int_{\mathbb{R}^{N}}\left|e^{i\langle u, \lambda\rangle}-\sum_{k=0}^{n} a_{k} e^{i\left\langle t^{k}, \lambda\right\rangle}\right|^{2} f(\lambda) d \lambda \geq c_{3,2} r^{2},
$$

where $t^{0}=0$ and $a_{0}=-1+\sum_{k=1}^{n} a_{k}$.

Let $\delta(\cdot): \mathbb{R}^{N} \rightarrow[0,1]$ be a function in $C^{\infty}\left(\mathbb{R}^{N}\right)$ such that $\delta(0)=1$ and it vanishes outside the open ball $B_{\rho}(0,1)$ in the metric $\rho$. Denote by $\widehat{\delta}$ the Fourier transform of $\delta$. Then $\widehat{\delta}(\cdot) \in C^{\infty}\left(\mathbb{R}^{N}\right)$ as well and $\widehat{\delta}(\lambda)$ decays rapidly as $|\lambda| \rightarrow \infty$.

Let $E$ be the diagonal matrix with $H_{1}^{-1}, \ldots, H_{N}^{-1}$ on its diagonal and let $\delta_{r}(t)=r^{-Q} \delta\left(r^{-E} t\right)$. Then the inverse Fourier transform and a change of variables yield

$$
\delta_{r}(t)=(2 \pi)^{-N} \int_{\mathbb{R}^{N}} e^{-i\langle t, \lambda\rangle} \widehat{\delta}\left(r^{E} \lambda\right) d \lambda .
$$

Since $\min \left\{\rho\left(u, t^{k}\right): 0 \leq k \leq n\right\} \geq r$, we have $\delta_{r}\left(u-t^{k}\right)=0$ for $k=0,1, \ldots, n$. This and (3.7) 
together imply that

$$
\begin{aligned}
J & :=\int_{\mathbb{R}^{N}}\left(e^{i\langle u, \lambda\rangle}-\sum_{k=0}^{n} a_{k} e^{i\left\langle t^{k}, \lambda\right\rangle}\right) e^{-i\langle u, \lambda\rangle} \widehat{\delta}\left(r^{E} \lambda\right) d \lambda \\
& =(2 \pi)^{N}\left(\delta_{r}(0)-\sum_{k=0}^{n} a_{k} \delta_{r}\left(u-t^{k}\right)\right) \\
& =(2 \pi)^{N} r^{-Q} .
\end{aligned}
$$

On the other hand, by the Cauchy-Schwarz inequality and (3.5), we have

$$
\begin{aligned}
J^{2} & \leq \int_{\mathbb{R}^{N}}\left|e^{i\langle u, \lambda\rangle}-\sum_{k=0}^{n} a_{k} e^{i\left\langle t^{k}, \lambda\right\rangle}\right|^{2} f(\lambda) d \lambda \cdot \int_{\mathbb{R}^{N}} \frac{1}{f(\lambda)}\left|\widehat{\delta}\left(r^{E} \lambda\right)\right|^{2} d \lambda \\
& \leq \mathbb{E}\left(X(u)-\sum_{k=1}^{n} a_{k} X\left(t^{k}\right)\right)^{2} \cdot r^{-Q} \int_{\mathbb{R}^{N}} \frac{1}{f\left(r^{-E} \lambda\right)}|\widehat{\delta}(\lambda)|^{2} d \lambda \\
& \leq c \mathbb{E}\left(X(u)-\sum_{k=1}^{n} a_{k} X\left(t^{k}\right)\right)^{2} \cdot r^{-2 Q-2},
\end{aligned}
$$

where $c>0$ is a constant which may only depend on $H$ and $N$.

We square both sides of (3.8) and use (3.9) to obtain

$$
(2 \pi)^{2 N} r^{-2 Q} \leq c r^{-2 Q-2} \mathbb{E}\left(X(u)-\sum_{k=1}^{n} a_{k} X\left(t^{k}\right)\right)^{2} .
$$

Hence (3.6) holds. This finishes the proof of the theorem.

Given jointly Gaussian random variables $Z_{1}, \ldots, Z_{n}$, we $\operatorname{denote}$ by $\operatorname{det} \operatorname{Cov}\left(Z_{1}, \ldots, Z_{n}\right)$ the determinant of their covariance matrix. If $\operatorname{det} \operatorname{Cov}\left(Z_{1}, \ldots, Z_{n}\right)>0$, then we have the identity

$$
\frac{(2 \pi)^{n / 2}}{\operatorname{det} \operatorname{Cov}\left(Z_{1}, \ldots, Z_{n}\right)}=\int_{\mathbb{R}^{n}} \mathbb{E} \exp \left(-i \sum_{k=1}^{n} u_{k} Z_{k}\right) d u_{1} \cdots d u_{n}
$$

By using the fact that, for every $k$, the conditional distribution of $Z_{k}$ given $Z_{1}, \ldots, Z_{k-1}$ is still Gaussian with mean $\mathbb{E}\left(Z_{k} \mid Z_{1}, \ldots, Z_{k-1}\right)$ and variance $\operatorname{Var}\left(Z_{k} \mid Z_{1}, \ldots, Z_{k-1}\right)$, one can evaluate the integral in the right-hand side of (3.10) and thus verify the following formula:

$$
\operatorname{det} \operatorname{Cov}\left(Z_{1}, \ldots, Z_{n}\right)=\operatorname{Var}\left(Z_{1}\right) \prod_{k=2}^{n} \operatorname{Var}\left(Z_{k} \mid Z_{1}, \ldots, Z_{k-1}\right) .
$$

A little thought reveals that (3.11) still holds when $\operatorname{det} \operatorname{Cov}\left(Z_{1}, \ldots, Z_{n}\right)=0$. Note that the lefthand side of (3.11) is permutation invariant for $Z_{1}, \ldots, Z_{n}$, one can represent $\operatorname{det} \operatorname{Cov}\left(Z_{1}, \ldots, Z_{n}\right)$ in terms of the conditional variances in $n$ ! different ways.

Combined with (3.1) or (3.3), the identity (3.11) can be applied to estimate the joint distribution of the Gaussian random variables $X\left(t^{1}\right), \ldots, X\left(t^{n}\right)$, where $t^{1}, \ldots, t^{n} \in \mathbb{R}^{N}$. This is why the properties of strong local nondeterminism are not only essential in this paper, but will also be 
useful in studying self-intersection local times [see Meerschaert et al. (2007) for results on fractional Brownian sheets], exact Hausdorff measure of the sample paths and other related problems.

The following simple result will be needed in Section 8 .

Lemma 3.4 Let $X$ be a Gaussian random field satisfying Condition (C3') [resp., (C3)]. Then for all integers $n \geq 1$ and for all distinct points $t^{1}, \ldots, t^{n} \in[\varepsilon, 1]^{N}\left[\right.$ resp., all points $t^{1}, \ldots, t^{n} \in$ $[\varepsilon, 1]^{N}$ with distinct coordinates, i.e., $t_{i}^{k} \neq t_{j}^{l}$ when $\left.(i, k) \neq(j, l)\right]$, the Gaussian random variables $X\left(t^{1}\right), \ldots, X\left(t^{n}\right)$ are linearly independent.

Proof We assume Condition $\left(\mathrm{C} 3^{\prime}\right)$ holds and let $t^{1}, \ldots, t^{n} \in[\varepsilon, 1]^{N}$ be $n$ distinct points. Then it follows from (3.11) that $\operatorname{det} \operatorname{Cov}\left(X\left(t^{1}\right), \ldots, X\left(t^{n}\right)\right)>0$. This proves the lemma. Similar conclusion holds when Condition (C3) is satisfied.

\section{Modulus of continuity}

It is sufficient to consider real-valued Gaussian random fields. Ayache and Xiao (2005) established a sharp modulus of continuity (i.e., including the logarithmic correction) for fractional Brownian sheets as a consequence of their wavelet expansion for $B_{0}^{H}$. Since the wavelet method depends on the stochastic integral representation (2.4), it can not be easily applied to Gaussian random fields in general. In this section, we describe several ways to establish sharp modulus of continuity for all anisotropic Gaussian random fields satisfying Condition (C1). The first two methods, i.e., the extended Garsia-Rodemich-Rumsey continuity lemma and the minorization metric method of Kwapień and Rosiński (2004), can be applied to random fields which are not necessarily Gaussian. Hence they can be more convenient when applied to solutions of stochastic partial differential equations. The third method, which is based on Dudley's entropy theorem and the Gaussian isoperimetric inequality, provides a stronger result in the sense that the upper bound is a constant instead of a random variable [cf. (4.15)].

Theorem 4.1 is an extension of the well-known Garsia-Rodemich-Rumsey continuity lemma [Garsia et al. (1970)]. It follows from Theorem 2.1 of Funaki, Kikuchi and Potthoff (2006), which is slightly more general [because of its freedom in choosing the function $p$ ] than an analogous result of Arnold and Imkeller (1996). A similar result can also be found in Dalang, Khoshnevisan and Nualart (2007a).

For our purpose, we have formulated it in terms of the metric $\rho$ defined in (2.27). Let $T \subseteq \mathbb{R}^{N}$ be a fixed closed interval. For any $r>0$ and $s \in T$, recall that $B_{\rho}(s, r)=\{t \in T: \rho(t, s) \leq r\}$ denotes the closed ball (in $T$ ) with center $s$ and radius $r$ in the metric $\rho$.

Theorem 4.1 Suppose that $Y: T \rightarrow \mathbb{R}$ is a continuous mapping. If there exist two strictly increasing functions $\Psi$ and $p$ on $\mathbb{R}_{+}$with $\Psi(0)=p(0)=0$ and $\lim _{u \rightarrow \infty} \Psi(u)=\infty$ such that

$$
K:=\int_{T} \int_{T} \Psi\left(\frac{|Y(s)-Y(t)|}{p(\rho(s, t))}\right) d s d t<\infty .
$$

Then for all $s, t \in T$, we have

$$
|Y(s)-Y(t)| \leq 8 \max _{z \in\{s, t\}} \int_{0}^{\rho(s, t)} \Psi^{-1}\left(\frac{4 K}{\lambda_{N}\left(B_{\rho}(z, u)\right)^{2}}\right) \tilde{p}(d u),
$$


where $\tilde{p}(u)=p(4 u)$ for all $u \in \mathbb{R}_{+}$.

Applying Theorem 4.1, we prove the following theorem on the modulus of continuity of an anisotropic Gaussian random field.

Theorem 4.2 Let $X=\left\{X(t), t \in \mathbb{R}^{N}\right\}$ be a centered Gaussian field in $\mathbb{R}$ satisfying Condition (C1). Then, almost surely, there exists a random variable $A$ depending on $N$ and $\left(H_{1}, \ldots, H_{N}\right)$ only such that $A$ has finite moments of all orders and for all $s, t \in I$,

$$
|X(s)-X(t)| \leq A \rho(s, t) \sqrt{\log \left(1+\rho(s, t)^{-1}\right)} .
$$

Proof In Theorem 4.1, let $T=I$ and we choose the functions $\Psi(x)=\exp \left(\frac{x^{2}}{4 c_{2,3}}\right)-1$ and $p(x)=x$, where $c_{2,3}>0$ is the constant in (2.29). It follows from Condition $(\mathrm{C} 1)$ that the random variable $K$ in (4.1) has finite moments of all orders and

$$
\begin{aligned}
\mathbb{E}(K) & =\mathbb{E} \int_{I} \int_{I} \Psi\left(\frac{|X(s)-X(t)|}{\rho(s, t)}\right) d s d t \\
& \leq \int_{I} \int_{I} \mathbb{E} \Psi(c|\xi|) d s d t=c_{4,1}<\infty .
\end{aligned}
$$

In the above $\xi$ is a standard normal random variable. Note that $\Psi^{-1}(u)=\sqrt{4 c_{2,3} \log (1+u)}$ and $\lambda_{N}\left(B_{\rho}(z, u)\right) \asymp u^{Q}$ is independent of $z$. Hence by Theorem 4.1 we have

$$
\begin{aligned}
|X(s)-X(t)| & \leq c \int_{0}^{\rho(s, t)} \sqrt{\log \left(1+\frac{4 K}{u^{Q}}\right)} d u \\
& \leq A \rho(s, t) \sqrt{\log \left(1+\rho(s, t)^{-1}\right)}
\end{aligned}
$$

where $A$ is a random variable depending on $K$ and we can choose it so that $A \leq c \max \{1, \log K\}$. Thus all moments of $A$ are finite. This finishes the proof of Theorem 4.2.

Let $X=\{X(t), t \in T\}$ be a stochastic process defined on a separable metric space $(T, d)$ and let $\psi$ be a Young function [that is, $\psi$ is strictly increasing, convex and $\psi(0)=0$ ]. Recently, Kwapien and Rosiński (2004) investigated the following problem: When can one find an appropriate metric $\tau$ on $T$ such that the implication

$$
\sup _{s, t \in T} \mathbb{E} \psi\left(\frac{|X(s)-X(t)|}{d(s, t)}\right)<\infty \Rightarrow \sup _{s, t \in T} \frac{|X(s)-X(t)|}{\tau(s, t)}<\infty, \quad \text { a.s. }
$$

holds? Their results can be applied to derive sharp modulus of continuity for a large class of stochastic processes including Gaussian random fields [but not stable random fields].

Recall from Kwapień and Rosiński (2004) that a probability measure $m$ on $T$ is called a weakly majorizing measure relative to $\psi$ and the metric $d$ if for all $s, t \in T$,

$$
\int_{0}^{d(s, t)} \psi^{-1}\left(\frac{1}{m\left(B_{d}(s, r)\right)}\right) d r<\infty
$$


where $\psi^{-1}$ denotes the inverse function of $\psi$ and $B_{d}(s, r)=\{t \in T: d(t, s) \leq r\}$. For every weakly majorizing measure $m$, the "minorizing metric" $\tau=\tau_{\psi, d, m}$ on $T$ relative to $\psi, d$ and $m$ is defined as

$$
\tau(s, t)=\max \left\{\int_{0}^{d(s, t)} \psi^{-1}\left(\frac{1}{m\left(B_{d}(s, r)\right)}\right) d r, \int_{0}^{d(t, s)} \psi^{-1}\left(\frac{1}{m\left(B_{d}(t, r)\right)}\right) d r\right\} .
$$

The following theorem of Kwapień and Rosiński (2004) gives a sufficient condition for (4.6) to hold.

Theorem 4.3 Let $\psi$ be a Young function satisfying the following growth condition:

$$
\psi(x) \psi(y) \leq \psi\left(c_{4,2}(x+y)\right) \quad \text { for all } x, y \geq 0,
$$

where $c_{4,2}>0$ is a constant. Let $m$ be a weakly majorizing measure relative to $\psi$ and $d$ on $T$. Then there exists a positive constant $c_{4,3}$ depending only on $\psi$ such that for every stochastic process $X=\{X(t), t \in T\}$,

$$
\mathbb{E} \psi\left(c_{4,3} \sup _{s, t \in T} \frac{|X(s)-X(t)|}{\tau(s, t)}\right) \leq 1+\sup _{s, t \in T} \mathbb{E} \psi\left(\frac{|X(s)-X(t)|}{d(s, t)}\right),
$$

where $\tau$ is the minorizing metric relative to $\psi, d$ and $m$.

Note that, for any $\alpha>0, \psi(x)=x^{\alpha}$ does not satisfy the growth condition (4.9), hence Theorem 4.3 is not applicable to stable random fields.

By applying Theorem 4.3 to the metric space $(I, \rho)$ in our setting, we can provide more information about the random variable $A$ in Theorem 4.2 .

Corollary 4.4 Let $X=\left\{X(t), t \in \mathbb{R}^{N}\right\}$ be a centered Gaussian field in $\mathbb{R}$ satisfying Condition (C1). Then there exists a constant $c_{4,4}>0$ such that

$$
\mathbb{E} \exp \left(c_{4,4} \sup _{s, t \in I} \frac{|X(s)-X(t)|^{2}}{\rho^{2}(s, t) \log \left(1+\rho(s, t)^{-1}\right)}\right)<\infty .
$$

Proof This can be verified by showing that the Lebesgue measure on $I$ is a weakly majorizing measure relative to the Young function $\psi(x)=e^{x^{2}}-1$ and the metric $\rho$; and the corresponding minorizing metric $\tau(s, t)$ satisfies

$$
c_{4,5} \rho(s, t) \sqrt{\log \left(1+\rho(s, t)^{-1}\right)} \leq \tau(s, t) \leq c_{4,6} \rho(s, t) \sqrt{\log \left(1+\rho(s, t)^{-1}\right)},
$$

for all $s, t \in I$. We leave the details to an interested reader. 
As a third method, we mention that it is also possible to obtain a uniform modulus of continuity for a Gaussian random field satisfying Condition (C1) by using the Gaussian isoperimetric inequality [cf. Lemma 2.1 in Talagrand (1995)]. To this end, we introduce an auxiliary Gaussian random field $Y=\{Y(s, t): t \in I, s \in[0, h]\}$ defined by $Y(t, s)=X(t+s)-X(t)$, where $h \in(0,1)^{N}$. Then the canonical metric $d$ on $T:=I \times[0, h]$ associated with $Y$ satisfies the following inequality:

$$
d\left((t, s),\left(t^{\prime}, s^{\prime}\right)\right) \leq c \min \left\{\rho(0, s)+\rho\left(0, s^{\prime}\right), \rho\left(s, s^{\prime}\right)+\rho\left(t, t^{\prime}\right)\right\} .
$$

Denote the $d$-diameter of $T$ by $D$. It follows from (4.12) that $D \leq c_{4,7} \sum_{j=1}^{N} h_{j}^{H_{j}}=c_{4,7} \rho(0, h)$, and the $d$-covering number of $T$ satisfies

$$
N_{d}(T, \varepsilon) \leq c\left(\frac{1}{\varepsilon}\right)^{Q} \prod_{j=1}^{N}\left(\frac{h_{j}}{\varepsilon^{1 / H_{j}}}\right) \leq c_{4,8} \varepsilon^{-2 Q} .
$$

One can verify that

$$
\int_{0}^{D} \sqrt{\log N_{d}(T, \varepsilon)} d \varepsilon \leq c_{4,9} \rho(0, h) \sqrt{\log \left(1+\rho(0, h)^{-1}\right)} .
$$

It follows from Lemma 2.1 in Talagrand (1995) that for all $u \geq 2 c_{4,9} \rho(0, h) \sqrt{\log \left(1+\rho(0, h)^{-1}\right)}$,

$$
\mathbb{P}\left\{\sup _{(t, s) \in T}|X(t+s)-X(t)| \geq u\right\} \leq \exp \left(-\frac{u^{2}}{D^{2}}\right)
$$

By using (4.14) and a standard Borel-Cantelli argument, we can prove that

$$
\limsup _{|h| \rightarrow 0} \frac{\sup _{t \in I, s \in[0, h]}|X(t+s)-X(t)|}{\rho(0, h) \sqrt{\log \left(1+\rho(0, h)^{-1}\right)}} \leq c_{4,10},
$$

where $c_{4,10}>0$ is a finite constant depending on $c_{2,4}, I$ and $H$ only.

We believe that, for Gaussian random fields satisfying $(\mathrm{C} 1)$, the rate function in (4.3) is sharp. This has been partly verified by Meerschaert, Wang and Xiao (2007) who proved that, if a Gaussian field $X$ satisfies Conditions (C1) and (C3), then

$$
c_{4,11} \leq \limsup _{|h| \rightarrow 0} \frac{\sup _{t \in I, s \in[0, h]}|X(s)-X(t)|}{\rho(0, h) \sqrt{\log \left(1+\rho(0, h)^{-1}\right)}} \leq c_{4,12},
$$

where $c_{4,11}$ and $c_{4,12}$ are positive constants depending on $c_{2,3}, c_{2,4}, I$ and $H$ only.

On the other hand, we can also use the above metric entropy method to prove that, for all $t_{0} \in I$ and $u>0$ large enough,

$$
\mathbb{P}\left\{\sup _{s \in[0, h]}\left|X\left(t_{0}+s\right)-X\left(t_{0}\right)\right| \geq \rho(0, h) u\right\} \leq \exp \left(-c_{4,13} u^{2}\right),
$$

where $c_{4,13}$ is a positive constant depending on $c_{2,4}, I$ and $H$ only. 
By using (4.17) and the Borel-Cantelli lemma, we derive the following local modulus of continuity for Gaussian random fields satisfying $(\mathrm{C} 1)$ : There exists a positive constant $c_{4,14}$ such that for every $t_{0} \in I$,

$$
\limsup _{|h| \rightarrow 0} \frac{\sup _{s \in[0, h]}\left|X\left(t_{0}+s\right)-X\left(t_{0}\right)\right|}{\rho(0, h) \sqrt{\log \log \left(1+\rho(0, h)^{-1}\right)}} \leq c_{4,14}, \quad \text { a.s. }
$$

Under certain mild conditions, it can be shown that (4.18) is sharp. For example, Meerschaert, Wang and Xiao (2007) proved that, if $X$ is a Gaussian random field with stationary increments and satisfies $(\mathrm{C} 1)$, then for every $t_{0} \in I$,

$$
\limsup _{|h| \rightarrow 0} \frac{\sup _{s \in[0, h]}\left|X\left(t_{0}+s\right)-X\left(t_{0}\right)\right|}{\rho(0, h) \sqrt{\log \log \left(1+\rho(0, h)^{-1}\right)}}=c_{4,15}, \quad \text { a.s. }
$$

where $c_{4,15}$ is a positive constant.

We should mention that one can also study the uniform and local moduli of continuity in terms of the increments of $X$ over intervals. Related results of this type for fractional Brownian sheets have been obtained by Wang (2007).

In the special case when $X$ is a direct sums of independent fractional Brownian motions of indices $H_{1}, \ldots, H_{N}$, that is,

$$
X(t)=X_{1}\left(t_{1}\right)+\cdots+X_{N}\left(t_{N}\right), \quad \forall t=\left(t_{1}, \ldots, t_{N}\right) \in \mathbb{R}^{N},
$$

where $X_{1}, \ldots, X_{N}$ are independent fractional Brownian motions in $\mathbb{R}$ of indices $H_{1}, \ldots, H_{N}$, respectively, Kôno (1975) established integral tests for the uniform and local upper and lower classes. It is natural to ask whether his results hold for more general anisotropic Gaussian random fields.

\section{$5 \quad$ Small ball probabilities}

In recent years, there has been much interest in studying the small ball probabilities of Gaussian processes. We refer to Li and Shao (2001) and Lifshits (1999) for extensive surveys on small ball probabilities and their applications. Small ball properties of fractional Brownian sheets have been considered by Dunker (2000), Mason and Shi (2001), Belinski and Linde (2002).

The small ball behavior of operator-scaling Gaussian random fields with stationary increments and the solution to the stochastic heat equation is different, as shown by the following general result.

Theorem 5.1 Let $X=\left\{X(t), t \in \mathbb{R}^{N}\right\}$ be a centered Gaussian field in $\mathbb{R}$ satisfying Conditions (C1) and $\left(C 3^{\prime}\right)$ on $I=[0,1]^{N}$. Then there exist positive constants $c_{5,1}$ and $c_{5,2}$ such that for all $\varepsilon>0$

$$
\exp \left(-\frac{c_{5,1}}{\varepsilon^{Q}}\right) \leq \mathbb{P}\left\{\max _{t \in[0,1]^{N}}|X(t)| \leq \varepsilon\right\} \leq \exp \left(-\frac{c_{5,2}}{\varepsilon^{Q}}\right),
$$

where $Q=\sum_{j=1}^{N} \frac{1}{H_{j}}$.

In order to prove the lower bound in (5.1), we will make use of the following general result of Talagrand (1993), see also Ledoux (1996, p.257). 
Lemma 5.2 Let $Y=\{Y(t), t \in T\}$ be a real-valued Gaussian process with mean zero and let $d$ be the canonical metric on $T$ defined by

$$
d(s, t)=\left(\mathbb{E}|Y(s)-Y(t)|^{2}\right)^{1 / 2}, \quad s, t \in T
$$

and denote by $N_{d}(T, \varepsilon)$ the smallest number of d-balls of radius $\varepsilon>0$ needed to cover $T$. Assume that there is a nonnegative function $\psi$ on $\mathbb{R}_{+}$such that $N_{d}(T, \varepsilon) \leq \psi(\varepsilon)$ for $\varepsilon>0$ and such that

$$
c_{5,3} \psi(\varepsilon) \leq \psi\left(\frac{\varepsilon}{2}\right) \leq c_{5,4} \psi(\varepsilon)
$$

for some constants $1<c_{5,3} \leq c_{5,4}<\infty$ and all $\varepsilon>0$. Then there is a constant $c_{5,5}>0$ such that

$$
\mathbb{P}\left\{\sup _{t, s \in T}|Y(t)-Y(s)| \leq \varepsilon\right\} \geq \exp \left(-c_{5,5} \psi(\varepsilon)\right) .
$$

Proof of Theorem 5.1 It follows from (C1) that for all $\varepsilon \in(0,1)$,

$$
N_{\rho}(I, \varepsilon) \leq c \varepsilon^{-Q}:=\psi(\varepsilon) .
$$

Clearly $\psi(\varepsilon)$ satisfies the condition (5.2). Hence the lower bound in (5.1) follows from Lemma 5.2.

The proof of the upper bound in (5.1) is based on Condition $\left(\mathrm{C} 3^{\prime}\right)$ and a conditioning argument in Monrad and Rootzén (1995). For any integer $n \geq 2$, we divide $[0,1]^{N}$ into $n^{Q}$ rectangles of side-lengths $n^{-1 / H_{j}}(j=1, \ldots, N)$. We denote the lower-left vertices of these rectangles (in any order) by $t_{n, k}\left(k=1,2, \ldots, n^{Q}\right)$. Then

$$
\mathbb{P}\left\{\max _{t \in[0,1]^{N}}|X(t)| \leq \varepsilon\right\} \leq \mathbb{P}\left\{\max _{1 \leq k \leq n}\left|X\left(t_{n, k}\right)\right| \leq \varepsilon\right\} .
$$

It follows from Condition $\left(\mathrm{C} 3^{\prime}\right)$ that for every $1 \leq k \leq n^{Q}$,

$$
\operatorname{Var}\left(X\left(t_{n, k}\right) \mid X\left(t_{n, i}\right), 1 \leq i \leq k-1\right) \geq c n^{-1} .
$$

This and Anderson's inequality for Gaussian measures imply the following upper bound for the conditional probabilities

$$
\mathbb{P}\left\{\left|X\left(t_{n, k}\right)\right| \leq \varepsilon \mid X\left(t_{n, j}\right), 1 \leq j \leq k-1\right\} \leq \Phi(c \varepsilon n),
$$

where $\Phi(x)$ is the distribution function of a standard normal random variable. It follows from (5.4) and (5.6) that

$$
\mathbb{P}\left\{\max _{t \in[0,1]^{N}}|X(t)| \leq \varepsilon\right\} \leq[\Phi(c \varepsilon n)]^{n^{Q}} .
$$

By taking $n=(c \varepsilon)^{-1}$, we obtain the upper bound in (5.1).

Remark 5.3 If $H_{1}=H_{2}=\cdots=H_{N}$, then (5.1) is of the same form as the small ball probability estimates for multiparameter fractional Brownian motion; see Talagrand (1995), Li and Shao (2001).

Among many applications, Theorem 5.1 can be applied to establish Chung-type laws of the iterated logarithm for anisotropic Gaussian random fields. Moreover, it would also be interesting to investigate the small ball probabilities of $X$ in other norms such as the $L^{2}$ or Hölder norms. 


\section{Hausdorff and packing dimensions of the range and graph}

In this section, we study the Hausdorff and packing dimensions of the range $X\left([0,1]^{N}\right)=\{X(t)$ : $\left.t \in[0,1]^{N}\right\}$ and the graph $\operatorname{Gr} X\left([0,1]^{N}\right)=\left\{(t, X(t)): t \in[0,1]^{N}\right\}$ of a Gaussian random field $X$ satisfying Condition $(\mathrm{C} 1)$ on $[0,1]^{N}$.

Hausdorff dimension and Hausdorff measure have been extensively used in describing thin sets and fractals. For any set $E \subseteq \mathbb{R}^{d}$ and $\gamma>0$, we will denote the Hausdorff dimension and the $\gamma$-dimensional Hausdorff measure of $E$ by $\operatorname{dim}_{\mathcal{H}} E$ and $\mathcal{H}^{\gamma}(E)$, respectively. We refer to Kahane (1985), Falconer (1990) or Mattila (1995) for their definitions and properties. More generally, for any nondecreasing, right continuous function $\varphi:[0,1] \rightarrow[0, \infty)$ with $\varphi(0)=0$, one can define the Hausdorff measure of $E$ with respect to $\varphi$ and denoted it by $\mathcal{H}^{\varphi}(E)$. We say that a function $\varphi$ is an exact Hausdorff measure function for $E$ if $0<\mathcal{H}^{\varphi}(E)<\infty$.

Now we recall briefly the definition of capacity and its connection to Hausdorff dimension. A kernel $\kappa$ is a measurable function $\kappa: \mathbb{R}^{d} \times \mathbb{R}^{d} \rightarrow[0, \infty]$. For a Borel measure $\mu$ on $\mathbb{R}^{d}$, the energy of $\mu$ with respect to the kernel $\kappa$ is defined by

$$
\mathcal{E}_{\kappa}(\mu)=\int_{\mathbb{R}^{d}} \int_{\mathbb{R}^{d}} \kappa(x, y) \mu(d x) \mu(d y) .
$$

For any Borel set $E \subseteq \mathbb{R}^{d}$, the capacity of $E$ with respect to $\kappa$, denoted by $\mathcal{C}_{\kappa}(E)$, is defined by

$$
\mathcal{C}_{\kappa}(E)=\left[\inf _{\mu \in \mathcal{P}(E)} \mathcal{E}_{\kappa}(\mu)\right]^{-1}
$$

where $\mathcal{P}(E)$ is the family of probability measures carried by $E$, and, by convention, $\infty^{-1}=0$. Note that $\mathcal{C}_{\kappa}(E)>0$ if and only if there is a probability measure $\mu$ on $E$ with finite $\kappa$-energy. We will mostly consider the case when $\kappa(x, y)=f(|x-y|)$, where $f$ is a non-negative and non-increasing function on $\mathbb{R}_{+}$. In particular, if

$$
f(r)= \begin{cases}r^{-\alpha} & \text { if } \alpha>0, \\ \log \left(\frac{e}{r \wedge 1}\right) & \text { if } \alpha=0,\end{cases}
$$

then the corresponding $\mathcal{E}_{\kappa}$ and $\mathcal{C}_{\kappa}$ will be denoted by $\mathcal{E}_{\alpha}$ and $\mathcal{C}_{\alpha}$, respectively; and the latter will be called the Bessel-Riesz capacity of order $\alpha$. The capacity dimension of $E$ is defined by

$$
\operatorname{dim}_{c}(E)=\sup \left\{\alpha>0: \mathcal{C}_{\alpha}(E)>0\right\}
$$

The well-known Frostman's theorem [cf. Kahane (1985, p.133) or Khoshnevisan (2002)] states that $\operatorname{dim}_{\mathcal{H}} E=\operatorname{dim}_{c}(E)$ for every compact set $E$ in $\mathbb{R}^{d}$. This result provides a very useful analytic way for the lower bound calculation of Hausdorff dimension. That is, for $E \subseteq \mathbb{R}^{d}$ in order to show $\operatorname{dim}_{\mathcal{H}} E \geq \alpha$, one only needs to find a measure $\mu$ on $E$ such that the $\alpha$-energy of $\mu$ is finite. For many deterministic and random sets such as self-similar sets or the range of a stochastic process, there are natural choices of $\mu$. This argument is usually referred to as the capacity argument.

Packing dimension and packing measure were introduced by Tricot (1982) and Taylor and Tricot (1985) as dual concepts to Hausdorff dimension and Hausdorff measure. We only recall briefly a definition of packing dimension, which will be denoted by $\operatorname{dim}_{\mathcal{P}}$. For any $\varepsilon>0$ and any bounded 
set $F \subseteq \mathbb{R}^{d}$, let $N(F, \varepsilon)$ be the smallest number of balls of radius $\varepsilon$ [in Euclidean metric] needed to cover $F$. Then the upper box-counting dimension of $F$ is defined as

$$
\overline{\operatorname{dim}}_{\mathcal{B}} F=\limsup _{\varepsilon \rightarrow 0} \frac{\log N(F, \varepsilon)}{-\log \varepsilon} .
$$

The packing dimension of $F$ can be defined by

$$
\operatorname{dim}_{\mathcal{P}} F=\inf \left\{\sup _{n} \overline{\operatorname{dim}}_{\mathcal{B}} F_{n}: \quad F \subseteq \bigcup_{n=1}^{\infty} F_{n}\right\}
$$

It is known that for any bounded set $F \subseteq \mathbb{R}^{d}$,

$$
\operatorname{dim}_{\mathcal{H}} F \leq \operatorname{dim}_{\mathcal{P}} F \leq \overline{\operatorname{dim}}_{\mathcal{B}} F \leq d .
$$

Further information on packing dimension and packing measure can be found in Falconer (1990) and Mattila (1995). We mention that various tools from fractal geometry have been applied to studying sample path properties of stochastic processes since 1950's. The survey papers of Taylor (1986) and Xiao (2004) summarize various fractal properties of random sets related to sample paths of Markov processes.

Throughout the rest of this paper, we will assume that

$$
0<H_{1} \leq \ldots \leq H_{N}<1
$$

Theorem 6.1 Let $X=\left\{X(t), t \in \mathbb{R}^{N}\right\}$ be an $(N, d)$-Gaussian field satisfying Condition (C1) on $I=[0,1]^{N}$. Then, with probability 1 ,

$$
\operatorname{dim}_{\mathcal{H}} X\left([0,1]^{N}\right)=\operatorname{dim}_{\mathcal{P}} X\left([0,1]^{N}\right)=\min \left\{d ; \quad \sum_{j=1}^{N} \frac{1}{H_{j}}\right\}
$$

and

$$
\begin{aligned}
& \operatorname{dim}_{\mathcal{H}} \operatorname{Gr} X\left([0,1]^{N}\right)=\operatorname{dim}_{\mathcal{P}} \operatorname{Gr} X\left([0,1]^{N}\right) \\
& =\min \left\{\sum_{j=1}^{k} \frac{H_{k}}{H_{j}}+N-k+\left(1-H_{k}\right) d, 1 \leq k \leq N ; \sum_{j=1}^{N} \frac{1}{H_{j}}\right\} \\
& \quad= \begin{cases}\sum_{j=1}^{N} \frac{1}{H_{j}}, & \text { if } \sum_{j=1}^{N} \frac{1}{H_{j}} \leq d, \\
\sum_{j=1}^{k} \frac{H_{k}}{H_{j}}+N-k+\left(1-H_{k}\right) d, & \text { if } \sum_{j=1}^{k-1} \frac{1}{H_{j}} \leq d<\sum_{j=1}^{k} \frac{1}{H_{j}},\end{cases}
\end{aligned}
$$

where $\sum_{j=1}^{0} \frac{1}{H_{j}}:=0$.

The last equality in (6.7) is verified by the following lemma, whose proof is elementary and is omitted. Denote

$$
\kappa:=\min \left\{\sum_{j=1}^{k} \frac{H_{k}}{H_{j}}+N-k+\left(1-H_{k}\right) d, 1 \leq k \leq N ; \sum_{j=1}^{N} \frac{1}{H_{j}}\right\} .
$$


Lemma 6.2 Assume (6.5) holds. We have

(i) If $d \geq \sum_{j=1}^{N} \frac{1}{H_{j}}$, then $\kappa=\sum_{j=1}^{N} \frac{1}{H_{j}}$.

(ii) If $\sum_{j=1}^{\ell-1} \frac{1}{H_{j}} \leq d<\sum_{j=1}^{\ell} \frac{1}{H_{j}}$ for some $1 \leq \ell \leq N$, then

$$
\kappa=\sum_{j=1}^{\ell} \frac{H_{\ell}}{H_{j}}+N-\ell+\left(1-H_{\ell}\right) d
$$

and $\kappa \in(N-\ell+d, N-\ell+d+1]$.

Because of (6.4) we can divide the proof of Theorem 6.1 into proving the upper bounds for the upper box dimensions and the lower bounds for the Hausdorff dimensions separately. The proofs are similar to those in Ayache and Xiao (2005) for fractional Brownian sheets. In the following, we first show that the upper bounds for $\overline{\operatorname{dim}}_{\mathcal{B}} X\left([0,1]^{N}\right)$ and $\overline{\operatorname{dim}}_{\mathcal{B}} \operatorname{Gr} X\left([0,1]^{N}\right)$ follow from Theorem 4.2 and a covering argument.

Proof of the upper bounds in Theorem 6.1. In order to prove the upper bound in (6.6), we note that clearly $\overline{\operatorname{dim}}_{\mathcal{B}} X\left([0,1]^{N}\right) \leq d$ a.s., so it suffices to prove the following inequality:

$$
\overline{\operatorname{dim}}_{\mathcal{B}} X\left([0,1]^{N}\right) \leq \sum_{j=1}^{N} \frac{1}{H_{j}}, \quad \text { a.s. }
$$

For any constants $0<\gamma_{j}<H_{j}(1 \leq j \leq N)$, it follows from Theorem 4.2 that there is a random variable $A$ of finite moments of all orders such that for almost all $\omega \in \Omega$,

$$
\sup _{s, t \in[0,1]^{N}} \frac{|X(s, \omega)-X(t, \omega)|}{\sum_{j=1}^{N}\left|s_{j}-t_{j}\right|^{\gamma_{j}}} \leq A(\omega) .
$$

We fix an $\omega$ such that (6.10) holds and then suppress it. For any integer $n \geq 2$, we divide $[0,1]^{N}$ into $m_{n}$ sub-rectangles $\left\{R_{n, i}\right\}$ with sides parallel to the axes and side-lengths $n^{-1 / H_{j}}(j=1, \ldots, N)$, respectively. Then

$$
m_{n} \leq c_{6,1} n^{\sum_{j=1}^{N} \frac{1}{H_{j}}}
$$

and $X\left([0,1]^{N}\right)$ can be covered by $X\left(R_{n, i}\right)\left(1 \leq i \leq m_{n}\right)$. By $(6.10)$, we see that the diameter of the image $X\left(R_{n, i}\right)$ satisfies

$$
\operatorname{diam} X\left(R_{n, i}\right) \leq c_{6,2} n^{-1+\delta},
$$

where $\delta=\max \left\{\left(H_{j}-\gamma_{j}\right) / H_{j}, 1 \leq j \leq N\right\}$. Consequently, for $\varepsilon_{n}=c_{6,2} n^{-1+\delta}, X\left([0,1]^{N}\right)$ can be covered by at most $m_{n}$ balls in $\mathbb{R}^{d}$ of radius $\varepsilon_{n}$. That is,

$$
N\left(X\left([0,1]^{N}\right), \varepsilon_{n}\right) \leq c_{6,1} n^{\sum_{j=1}^{N} \frac{1}{H_{j}}} .
$$

This implies

$$
\overline{\operatorname{dim}}_{\mathcal{B}} X\left([0,1]^{N}\right) \leq \frac{1}{1-\delta} \sum_{j=1}^{N} \frac{1}{H_{j}}, \quad \text { a.s. }
$$


By letting $\gamma_{j} \uparrow H_{j}$ along rational numbers, we have $\delta \downarrow 0$ and (6.9) follows from (6.14).

Now we turn to the proof of the upper bound in (6.7). We will show that there are several different ways to cover $\operatorname{Gr} X\left([0,1]^{N}\right)$ by balls in $\mathbb{R}^{N+d}$ of the same radius, each of which leads to an upper bound for $\overline{\operatorname{dim}}_{\mathcal{B}} \operatorname{Gr} X\left([0,1]^{N}\right)$.

For each fixed integer $n \geq 2$, we have

$$
\operatorname{Gr} X\left([0,1]^{N}\right) \subseteq \bigcup_{i=1}^{m_{n}} R_{n, i} \times X\left(R_{n, i}\right) .
$$

It follows from (6.12) and (6.15) that $\operatorname{Gr} X\left([0,1]^{N}\right)$ can be covered by $m_{n}$ balls in $\mathbb{R}^{N+d}$ with radius $c_{6,2} n^{-1+\delta}$ and the same argument as the above yields

$$
\overline{\operatorname{dim}}_{\mathcal{B}} \operatorname{Gr} X\left([0,1]^{N}\right) \leq \sum_{j=1}^{N} \frac{1}{H_{j}}, \quad \text { a.s. }
$$

We fix an integer $1 \leq k \leq N$. Observe that each $R_{n, i} \times X\left(R_{n, i}\right)$ can be covered by $\ell_{n, k}$ balls (or cubes) in $\mathbb{R}^{N+d}$ of radius (or side-length) $n^{-\frac{1}{H_{k}}}$, where by (6.10) we have

$$
\ell_{n, k} \leq c n^{\sum_{j=k}^{N}\left(\frac{1}{H_{k}}-\frac{1}{H_{j}}\right)} \times n^{\left(\frac{1}{H_{k}}-1+\delta\right) d}, \quad \text { a.s. }
$$

Hence $\operatorname{Gr} X\left([0,1]^{N}\right)$ can be covered by $m_{n} \times \ell_{n, k}$ balls in $\mathbb{R}^{N+d}$ with radius $n^{-\frac{1}{H_{k}}}$. Consequently,

$$
\overline{\operatorname{dim}}_{\mathcal{B}} \operatorname{Gr} X\left([0,1]^{N}\right) \leq \sum_{j=1}^{k} \frac{H_{k}}{H_{j}}+N-k+\left(1-H_{k}+\delta H_{k}\right) d, \quad \text { a.s. }
$$

Letting $\gamma_{j} \uparrow H_{j}$ along rational numbers, we derive that for every $k=1, \ldots, N$,

$$
\overline{\operatorname{dim}}_{\mathcal{B}} \operatorname{Gr} X\left([0,1]^{N}\right) \leq \sum_{j=1}^{k} \frac{H_{k}}{H_{j}}+N-k+\left(1-H_{k}\right) d .
$$

Combining (6.16) and (6.18) yields the upper bound in (6.7).

For proving the lower bounds in Theorem 6.1, we will make use of the following elementary Lemmas 6.3 and 6.4. The former is proved in Xiao and Zhang (2002, p.212) which will be used to derive a lower bound for $\operatorname{dim}_{\mathcal{H}} X\left([0,1]^{N}\right)$; the latter is proved in Ayache and Xiao (2005) which will be needed for determining a lower bound for $\operatorname{dim}_{\mathcal{H}} \operatorname{Gr} X\left([0,1]^{N}\right)$. Both lemmas will be useful in the proof of Theorem 7.1 in Section 7.

Lemma 6.3 Let $0<\alpha<1$ and $\varepsilon>0$ be given constants. Then for any constants $\delta>2 \alpha, M>0$ and $p>0$, there exists a positive and finite constant $c_{6,3}$, depending on $\varepsilon, \delta, p$ and $M$ only, such that for all $0<A \leq M$,

$$
\int_{\varepsilon}^{1} d s \int_{\varepsilon}^{1} \frac{d t}{\left(A+|s-t|^{2 \alpha}\right)^{p}} \leq c_{6,3}\left(A^{-\left(p-\frac{1}{\delta}\right)}+1\right) .
$$


Lemma 6.4 Let $\alpha, \beta$ and $\eta$ be positive constants. For $A>0$ and $B>0$, let

$$
J:=J(A, B)=\int_{0}^{1} \frac{d t}{\left(A+t^{\alpha}\right)^{\beta}(B+t)^{\eta}} .
$$

Then there exist finite constants $c_{6,4}$ and $c_{6,5}$, depending on $\alpha, \beta, \eta$ only, such that the following hold for all real numbers $A, B>0$ satisfying $A^{1 / \alpha} \leq c_{6,3} B$ :

(i) If $\alpha \beta>1$, then

$$
J \leq c_{6,5} \frac{1}{A^{\beta-\alpha^{-1} B^{\eta}}}
$$

(ii) If $\alpha \beta=1$, then

$$
J \leq c_{6,5} \frac{1}{B^{\eta}} \log \left(1+B A^{-1 / \alpha}\right)
$$

(iii) If $0<\alpha \beta<1$ and $\alpha \beta+\eta \neq 1$, then

$$
J \leq c_{6,5}\left(\frac{1}{B^{\alpha \beta+\eta-1}}+1\right) .
$$

Proof of the lower bounds in Theorem 6.1. First we prove the lower bound in (6.6). Note that for any $\varepsilon \in(0,1), \operatorname{dim}_{\mathcal{H}} X\left([0,1]^{N}\right) \geq \operatorname{dim}_{\mathcal{H}} X\left([\varepsilon, 1]^{N}\right)$. It is sufficient to show that $\operatorname{dim}_{\mathcal{H}} X\left([\varepsilon, 1]^{N}\right) \geq$ $\gamma$ a.s. for every $0<\gamma<\min \left\{d, \sum_{j=1}^{N} \frac{1}{H_{j}}\right\}$.

Let $\mu_{X}$ be the image measure of the Lebesgue measure on $[\varepsilon, 1]^{N}$ under the mapping $t \mapsto X(t)$. Then the energy of $\mu_{X}$ of order $\gamma$ can be written as

$$
\int_{\mathbb{R}^{d}} \int_{\mathbb{R}^{d}} \frac{\mu_{X}(d x) \mu(d y)}{|x-y|^{\gamma}}=\int_{[\varepsilon, 1]^{N}} \int_{[\varepsilon, 1]^{N}} \frac{d s d t}{|X(s)-X(t)|^{\gamma}} .
$$

Hence by Frostman's theorem [see, e.g., Kahane (1985, Chapter 10)], it is sufficient to show that for every $0<\gamma<\min \left\{d, \sum_{j=1}^{N} \frac{1}{H_{j}}\right\}$,

$$
\mathcal{E}_{\gamma}=\int_{[\varepsilon, 1]^{N}} \int_{[\varepsilon, 1]^{N}} \mathbb{E}\left(\frac{1}{|X(s)-X(t)|^{\gamma}}\right) d s d t<\infty
$$

Since $0<\gamma<d$, we have $0<\mathbb{E}\left(|\Xi|^{-\gamma}\right)<\infty$, where $\Xi$ is a standard $d$-dimensional normal vector. Combining this fact with Condition (C1), we have

$$
\varepsilon_{\gamma} \leq c \int_{\varepsilon}^{1} d s_{1} \int_{\varepsilon}^{1} d t_{1} \cdots \int_{\varepsilon}^{1} d s_{N} \int_{\varepsilon}^{1} \frac{1}{\left(\sum_{j=1}^{N}\left|s_{j}-t_{j}\right|^{2 H_{j}}\right)^{\gamma / 2}} d t_{N} .
$$

We choose positive constants $\delta_{2}, \ldots, \delta_{N}$ such that $\delta_{j}>2 H_{j}$ for each $2 \leq j \leq N$ and

$$
\frac{1}{\delta_{2}}+\cdots+\frac{1}{\delta_{N}}<\frac{\gamma}{2}<\frac{1}{2 H_{1}}+\frac{1}{\delta_{2}}+\cdots+\frac{1}{\delta_{N}}
$$

This is possible since $\gamma<\sum_{j=1}^{N} \frac{1}{H_{j}}$. 
Applying Lemma 6.3 to (6.25) with

$$
A=\sum_{j=1}^{N-1}\left|s_{j}-t_{j}\right|^{2 H_{j}} \quad \text { and } p=\gamma / 2
$$

we obtain that

$$
\varepsilon_{\gamma} \leq c_{6,6}+c_{6,6} \int_{\varepsilon}^{1} d s_{1} \int_{\varepsilon}^{1} d t_{1} \cdots \int_{\varepsilon}^{1} d s_{N-1} \int_{\varepsilon}^{1} \frac{d t_{N-1}}{\left(\sum_{j=1}^{N-1}\left|s_{j}-t_{j}\right|^{2 H_{j}}\right)^{\gamma / 2-1 / \delta_{N}}}
$$

By repeatedly using Lemma 6.3 to the integral in (6.27) for $N-2$ steps, we derive that

$$
\varepsilon_{\gamma} \leq c_{6,7}+c_{6,7} \int_{\varepsilon}^{1} d s_{1} \int_{\varepsilon}^{1} \frac{d t_{1}}{\left(\left|s_{1}-t_{1}\right|^{2 H_{1}}\right)^{\gamma / 2-\left(1 / \delta_{2}+\cdots+1 / \delta_{N}\right)}}
$$

Since the $\delta_{j}$ 's satisfy $(6.26)$, we have $2 H_{1}\left[\gamma / 2-\left(1 / \delta_{2}+\cdots+1 / \delta_{N}\right)\right]<1$. Thus the integral in the right hand side of (6.28) is finite. This proves (6.24), and (6.6) follows.

Now we prove the lower bound in (6.7). Since $\operatorname{dim}_{\mathcal{H}} \operatorname{Gr} X\left([0,1]^{N}\right) \geq \operatorname{dim}_{\mathcal{H}} X\left([0,1]^{N}\right)$ always holds, we only need to consider the case when

$$
\sum_{j=1}^{k-1} \frac{1}{H_{j}} \leq d<\sum_{j=1}^{k} \frac{1}{H_{j}} \quad \text { for some } \quad 1 \leq k \leq N .
$$

Here and in the sequel, $\sum_{j=1}^{0} \frac{1}{H_{j}}=0$.

Let $0<\varepsilon<1$ and $0<\gamma<\sum_{j=1}^{k} \frac{H_{k}}{H_{j}}+N-k+\left(1-H_{k}\right) d$ be fixed, but arbitrary, constants. By Lemma 6.2, we may and will assume $\gamma \in(N-k+d, N-k+d+1)$. In order to prove $\operatorname{dim}_{\mathcal{H}} \operatorname{Gr} X\left([\varepsilon, 1]^{N}\right) \geq \gamma$ a.s., again by Frostman's theorem, it is sufficient to show

$$
\mathcal{G}_{\gamma}=\int_{[\varepsilon, 1]^{N}} \int_{[\varepsilon, 1]^{N}} \mathbb{E}\left[\frac{1}{\left(|s-t|^{2}+|X(s)-X(t)|^{2}\right)^{\gamma / 2}}\right] d s d t<\infty .
$$

Since $\gamma>d$, we note that for a standard normal vector $\Xi$ in $\mathbb{R}^{d}$ and any number $a \in \mathbb{R}$,

$$
\mathbb{E}\left[\frac{1}{\left(a^{2}+|\Xi|^{2}\right)^{\gamma / 2}}\right] \leq c_{6,8} a^{-(\gamma-d)}
$$

see, e.g., Kahane (1985, p.279). Consequently, we derive that

$$
\mathcal{G}_{\gamma} \leq c_{6,8} \int_{[\varepsilon, 1]^{N}} \int_{[\varepsilon, 1]^{N}} \frac{1}{\sigma(s, t)^{d}|s-t|^{\gamma-d}} d s d t
$$

where $\sigma^{2}(s, t)=\mathbb{E}\left[\left(X_{1}(s)-X_{1}(t)\right)^{2}\right]$. By Condition $(\mathrm{C} 1)$ and a change of variables, we have

$$
\mathcal{S}_{\gamma} \leq c_{6,9} \int_{0}^{1} d t_{N} \cdots \int_{0}^{1} \frac{1}{\left(\sum_{j=1}^{N} t_{j}^{H_{j}}\right)^{d}\left(\sum_{j=1}^{N} t_{j}\right)^{\gamma-d}} d t_{1} .
$$


In order to show the integral in (6.32) is finite, we will integrate $\left[d t_{1}\right], \ldots,\left[d t_{k}\right]$ iteratively. Furthermore, we will assume $k>1$ in (6.29) [If $k=1$, we can use (6.23) to obtain (6.36) directly].

We integrate $\left[d t_{1}\right]$ first. Since $H_{1} d>1$, we can use (6.21) of Lemma 6.4 with $A=\sum_{j=2}^{N} t_{j}^{H_{j}}$ and $B=\sum_{j=2}^{N} t_{j}$ to get

$$
\mathcal{S}_{\gamma} \leq c_{6,10} \int_{0}^{1} d t_{N} \cdots \int_{0}^{1} \frac{1}{\left(\sum_{j=2}^{N} t_{j}^{H_{j}}\right)^{d-1 / H_{1}}\left(\sum_{j=2}^{N} t_{j}\right)^{\gamma-d}} d t_{2} .
$$

We can repeat this procedure for integrating $d t_{2}, \ldots, d t_{k-1}$. Note that if $d=\sum_{j=1}^{k-1} \frac{1}{H_{j}}$, then we need to use (6.22) to integrate $\left[d t_{k-1}\right]$ and obtain

$$
\mathcal{G}_{\gamma} \leq c_{6,11} \int_{0}^{1} d t_{N} \cdots \int_{0}^{1} \frac{1}{\left(\sum_{j=k}^{N} t_{j}\right)^{\gamma-d}} \log \left(1+\frac{1}{\sum_{j=k}^{N} t_{j}}\right) d t_{k}<\infty .
$$

Note that the last integral is finite since $\gamma-d<N-k+1$. On the other hand, if $d>\sum_{j=1}^{k-1} \frac{1}{H_{j}}$, then (6.21) gives

$$
\mathcal{G}_{\gamma} \leq c_{6,12} \int_{0}^{1} d t_{N} \cdots \int_{0}^{1} \frac{d t_{k}}{\left(\sum_{j=k}^{N} t_{j}^{H_{j}}\right)^{d-\sum_{j=1}^{k-1} 1 / H_{j}}\left(\sum_{j=k}^{N} t_{j}\right)^{\gamma-d}} .
$$

We integrate $\left[d t_{k}\right]$ in (6.35) and by using (6.23), we see that

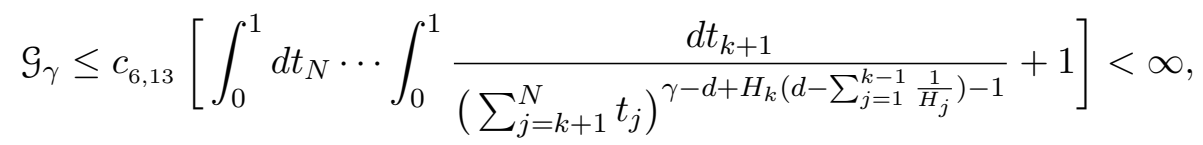

since $\gamma-d+H_{k}\left(d-\sum_{j=1}^{k-1} \frac{1}{H_{j}}\right)-1<N-k$. Combining (6.34) and (6.36) yields (6.30). This completes the proof of Theorem 6.1.

There are several possible ways to strengthen and extend Theorem 6.1. For example, it would be interesting to determine the exact Hausdorff and packing measure functions for the range $X\left([0,1]^{N}\right)$ and graph $\operatorname{Gr} X\left([0,1]^{N}\right)$ for anisotropic Gaussian random fields. When $X$ is the Brownian sheet or a fractional Brownian motion, the problems on exact Hausdorff measure functions have been considered by Ehm (1981), Talagrand (1995, 1998), Xiao (1996a, 1997a, 1997b). Here is a summary of the known results:

(i). Let $X^{\alpha}=\left\{X^{\alpha}(t), t \in \mathbb{R}^{N}\right\}$ be an $(N, d)$-fractional Brownian motion of index $\alpha$. If $N<$ $\alpha d$, then $\varphi_{1}(r)=r^{N / \alpha} \log \log 1 / r$ ia an exact Hausdorff measure function for the range and graph of $X^{\alpha}$. If $N>\alpha d$, then $X^{\alpha}\left([0,1]^{N}\right)$ a.s. has positive Lebesgue measure and interior points; and $\varphi_{2}(r)=r^{N+(1-\alpha) d}(\log \log 1 / r)^{\frac{\alpha d}{N}}$ is an exact Hausdorff measure function for the graph of $X^{\alpha}$. If $N=\alpha d$, then $\mathcal{H}^{\varphi_{3}}\left(X^{\alpha}\left([0,1]^{N}\right)\right)$ is $\sigma$-finite almost surely, where $\varphi_{3}(r)=$ $r^{d} \log (1 / r) \log \log \log 1 / r$. In the latter case the same is also true for the Hausdorff measure of the graph set of $X^{\alpha}(t)$. However, the lower bound problems for the Hausdorff measure of the range and graph remain open. 
(ii). Let $W=\left\{W(t), t \in \mathbb{R}_{+}^{N}\right\}$ be the Brownian sheet in $\mathbb{R}^{d}$. If $2 N<d$, then $\varphi_{4}(r)=$ $r^{2 N}(\log \log 1 / r)^{N}$ ia an exact Hausdorff measure function for the range and graph of $W$. If $2 N>d$, then $W\left([0,1]^{N}\right)$ a.s. has interior points and $\varphi_{5}(r)=r^{N+\frac{d}{2}}(\log \log 1 / r)^{\frac{d}{2}}$ is an exact Hausdorff measure function for the graph of $W$. When $2 N=d$, the problems for finding exact Hausdorff measure functions for the range and graph of $W$ are completely open.

It is interesting to notice the subtle differences in the exact Hausdorff functions for the range and graph sets of fractional Brownian motion and the Brownian sheet, respectively. I believe the differences are a reflection of the two different types of local nondeterminism that they satisfy.

We remark that the methods in the aforementioned references rely respectively on specific properties of the Brownian sheet and fractional Brownian motion, and it is not clear whether these methods are applicable to Gaussian random fields satisfying $(\mathrm{C} 3)$ or $\left(\mathrm{C} 3^{\prime}\right)$. It would be interesting to develop general methods that are applicable to larger classes of (Gaussian) random fields.

The problems on exact packing measure functions for $X\left([0,1]^{N}\right)$ and $\operatorname{Gr} X\left([0,1]^{N}\right)$ are related to the liminf properties of the occupation measures of $X$ and are more difficult to study. When $X$ is an $(N, d)$-fractional Brownian motion of index $\alpha$ and $N<\alpha d$, Xiao (1996b, 2003) proved that $\varphi_{6}(r)=r^{N / \alpha}(\log \log 1 / r)^{-N /(2 \alpha)}$ is an exact packing measure function for $X\left([0,1]^{N}\right)$ and $\operatorname{Gr} X\left([0,1]^{N}\right)$. For all the other Gaussian fields including the Brownian sheet, the corresponding problems remain to be open.

On the other hand, it is a natural question is to find $\operatorname{dim}_{\mathcal{H}} X(E)$ when $E \subseteq \mathbb{R}^{N}$ is an arbitrary Borel set, say a fractal set. It is not hard to see that, due to the anisotropy of $X$, the Hausdorff dimension of $X(E)$ can not be determined by $\operatorname{dim}_{\mathcal{H}} E$ and the index $H$ alone, as shown by Example 6.6 below. This is in contrast with the cases of fractional Brownian motion or the Brownian sheet.

We start with the following Proposition 6.5 which determines $\operatorname{dim}_{\mathcal{H}} X(E)$ when $E$ belongs to a special class of Borel sets in $\mathbb{R}^{N}$. Since the proof is almost the same as that of Proposition 3.1 in $\mathrm{Wu}$ and Xiao (2007), we omit the proof.

Proposition 6.5 Let $X=\left\{X(t), t \in \mathbb{R}^{N}\right\}$ be an $(N, d)$-Gaussian random field satisfying Condition (C1) on $I=[0,1]^{N}$ with parameters $\left(H_{1}, \ldots, H_{N}\right)$. Assume that $E_{j}(j=1, \ldots, N)$ are Borel subsets of $(0,1)$ satisfying the following property: $\exists\left\{j_{1}, \ldots, j_{N-1}\right\} \subseteq\{1, \ldots, N\}$ such that $\operatorname{dim}_{\mathcal{H}} E_{j_{k}}=$ $\operatorname{dim}_{\mathcal{P}} E_{j_{k}}$ for $k=1, \ldots, N-1$. Let $E=E_{1} \times \cdots \times E_{N} \subseteq \mathbb{R}^{N}$, then we have

$$
\operatorname{dim}_{\mathcal{H}} X(E)=\min \left\{d ; \quad \sum_{j=1}^{N} \frac{\operatorname{dim}_{\mathcal{H}} E_{j}}{H_{j}}\right\}, \quad \text { a.s. }
$$

The following simple example illustrates that, in general, $\operatorname{dim}_{\mathcal{H}} E$ alone is not enough to determine the Hausdorff dimension of $X(E)$.

Example 6.6 Let $X=\left\{X(t), t \in \mathbb{R}^{2}\right\}$ be a $(2, d)$-Gaussian field with index $H=\left(H_{1}, H_{2}\right)$ and $H_{1}<H_{2}$. Let $E=E_{1} \times E_{2}$ and $F=E_{2} \times E_{1}$, where $E_{1} \subseteq(0,1)$ satisfies $\operatorname{dim}_{\mathcal{H}} E_{1}=\operatorname{dim}_{\mathcal{P}} E_{1}$ and $E_{2} \subseteq(0,1)$ is arbitrary. It is well known that

$$
\operatorname{dim}_{\mathcal{H}} E=\operatorname{dim}_{\mathcal{H}} E_{1}+\operatorname{dim}_{\mathcal{H}} E_{2}=\operatorname{dim}_{\mathcal{H}} F,
$$

cf. Falconer (1990, p.94). However, by Proposition 6.5 we have

$$
\operatorname{dim}_{\mathcal{H}} X(E)=\min \left\{d ; \quad \frac{\operatorname{dim}_{\mathcal{H}} E_{1}}{H_{1}}+\frac{\operatorname{dim}_{\mathcal{H}} E_{2}}{H_{2}}\right\}
$$


and

$$
\operatorname{dim}_{\mathcal{H}} X(F)=\min \left\{d ; \quad \frac{\operatorname{dim}_{\mathcal{H}} E_{2}}{H_{1}}+\frac{\operatorname{dim}_{\mathcal{H}} E_{1}}{H_{2}}\right\} .
$$

We see that $\operatorname{dim}_{\mathcal{H}} X(E) \neq \operatorname{dim}_{\mathcal{H}} X(F)$ in general unless $\operatorname{dim}_{\mathcal{H}} E_{1}=\operatorname{dim}_{\mathcal{H}} E_{2}$.

Example 6.6 shows that for determining $\operatorname{dim}_{\mathcal{H}} X(E)$, we need to have more information about the geometry of $E$ than its Hausdorff dimension.

In order to solve the problem for finding the Hausdorff dimension of the image $B^{H}(E)$ of fractional Brownian sheet $B^{H}$, Wu and Xiao (2007) applied a measure-theoretic approach and introduced a notion of Hausdorff dimension contour for finite Borel measures and Borel sets.

Recall that the Hausdorff dimension of a Borel measure $\mu$ on $\mathbb{R}^{N}$ (or lower Hausdorff dimension as it is sometimes called) is defined by

$$
\operatorname{dim}_{\mathcal{H}} \mu=\inf \left\{\operatorname{dim}_{\mathcal{H}} F: \mu(F)>0 \text { and } F \subseteq \mathbb{R}^{N} \text { is a Borel set }\right\} .
$$

$\mathrm{Hu}$ and Taylor (1994) proved the following characterization of $\operatorname{dim}_{\mathcal{H}} \mu$ : If $\mu$ is a finite Borel measure on $\mathbb{R}^{N}$, then

$$
\operatorname{dim}_{\mathcal{H}} \mu=\sup \left\{\gamma \geq 0: \limsup _{r \rightarrow 0^{+}} \frac{\mu(B(t, r))}{r^{\gamma}}=0 \text { for } \mu \text {-a.e. } t \in \mathbb{R}^{N}\right\},
$$

where $B(t, r)=\left\{s \in \mathbb{R}^{N}:|s-t| \leq r\right\}$. It can be verified that for every Borel set $E \subseteq \mathbb{R}^{N}$, we have

$$
\operatorname{dim}_{\mathcal{H}} E=\sup \left\{\operatorname{dim}_{\mathcal{H}} \mu: \mu \in \mathcal{M}_{c}^{+}(E)\right\},
$$

where $\mathcal{M}_{c}^{+}(E)$ denotes the family of finite Borel measures on $E$ with compact support in $E$.

From (6.39), we note that $\operatorname{dim}_{\mathcal{H}} \mu$ only describes the local behavior of $\mu$ in an isotropic way and is not quite informative if $\mu$ is highly anisotropic. To overcome this difficulty, Wu and Xiao (2007) introduce the following notion of "dimension" for $E \subseteq(0, \infty)^{N}$ that is natural for studying $X(E)$.

Definition 6.7 Given a Borel probability measure $\mu$ on $\mathbb{R}^{N}$, we define the set $\Lambda_{\mu} \subseteq \mathbb{R}_{+}^{N}$ by

$$
\Lambda_{\mu}=\left\{\lambda=\left(\lambda_{1}, \ldots, \lambda_{N}\right) \in \mathbb{R}_{+}^{N}: \limsup _{r \rightarrow 0^{+}} \frac{\mu(R(t, r))}{r^{\left\langle\lambda, H^{-1}\right\rangle}}=0 \text { for } \mu \text {-a.e. } t \in \mathbb{R}^{N}\right\}
$$

where $R(t, r)=\prod_{j=1}^{N}\left[t_{j}-r^{1 / H_{j}}, t_{j}+r^{1 / H_{j}}\right]$ and $H^{-1}=\left(\frac{1}{H_{1}}, \ldots, \frac{1}{H_{N}}\right)$.

The following lemma is proved in Wu and Xiao (2007), which summarizes some basic properties of $\Lambda_{\mu}$. Recall that $H_{1}=\min \left\{H_{j}: 1 \leq j \leq N\right\}$.

Lemma $6.8 \Lambda_{\mu}$ has the following properties:

(i) The set $\Lambda_{\mu}$ is bounded:

$$
\Lambda_{\mu} \subseteq\left\{\lambda=\left(\lambda_{1}, \ldots, \lambda_{N}\right) \in \mathbb{R}_{+}^{N}:\left\langle\lambda, H^{-1}\right\rangle \leq \frac{N}{H_{1}}\right\}
$$

(ii) For all $\beta \in(0,1]^{N}$ and $\lambda \in \Lambda_{\mu}$, the Hadamard product of $\beta$ and $\lambda$, $\beta \circ \lambda=\left(\beta_{1} \lambda_{1}, \ldots, \beta_{N} \lambda_{N}\right) \in$ $\Lambda_{\mu}$. 
(iii) $\Lambda_{\mu}$ is convex, i.e. $\forall \lambda, \eta \in \Lambda_{\mu}$ and $0<b<1, b \lambda+(1-b) \eta \in \Lambda_{\mu}$.

(iv) For every $a \in(0, \infty)^{N}$, $\sup _{\lambda \in \Lambda_{\mu}}\langle\lambda, a\rangle$ is achieved on the boundary of $\Lambda_{\mu}$.

We call the boundary of $\Lambda_{\mu}$, denoted by $\partial \Lambda_{\mu}$, the Hausdorff dimension contour of $\mu$. See Wu and Xiao (2007) for some examples for determining $\partial \Lambda_{\mu}$.

For any Borel set $E \subseteq \mathbb{R}^{N}$, we define

$$
\Lambda(E)=\bigcup_{\mu \in \mathcal{M}_{c}^{+}(E)} \Lambda_{\mu}
$$

Similar to the case for measures, we call the set $\cup_{\mu \in \mathcal{M}_{c}^{+}(E)} \partial \Lambda_{\mu}$ the Hausdorff dimension contour

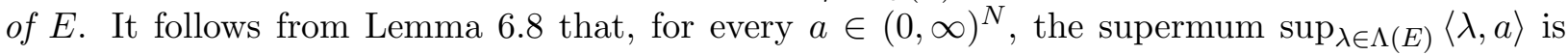
determined by the Hausdorff dimension contour of $E$.

The same proof of Theorem 3.10 in $\mathrm{Wu}$ and Xiao (2007) yields the following result.

Theorem 6.9 Let $X=\left\{X(t), t \in \mathbb{R}^{N}\right\}$ be an $(N, d)$-Gaussian random field satisfying Condition (C1) on $I=[0,1]^{N}$. Then for every Borel set $E \subseteq[0,1]^{N}$,

$$
\operatorname{dim}_{\mathcal{H}} X(E)=\min \{d, s(H, E)\} \quad \text { a.s., }
$$

where $s(H, E)=\sup _{\lambda \in \Lambda(E)}\left\langle\lambda, H^{-1}\right\rangle=\sup _{\mu \in \mathcal{M}_{c}^{+}(E)} s_{\mu}(E)$.

In the following, we give a more direct approach. Our results yield more geometric information about the quantity $s(H, E)$ as well.

For an arbitrary vector $\left(H_{1}, \ldots, H_{N}\right) \in(0,1)^{N}$, we consider the metric space $\left(\mathbb{R}^{N}, \rho\right)$, where $\rho$ is defined by (2.27). For any $\beta>0$ and $E \subseteq \mathbb{R}^{N}$, define the $\beta$-dimensional Hausdorff measure [in the metric $\rho$ ] of $E$ by

$$
\mathcal{H}_{\rho}^{\beta}(E)=\lim _{\delta \rightarrow 0} \inf \left\{\sum_{n=1}^{\infty}\left(2 r_{n}\right)^{\beta}: E \subseteq \bigcup_{n=1}^{\infty} B_{\rho}\left(r_{n}\right), r_{n} \leq \delta\right\}
$$

This is a metric outer measure and all Borel sets are $\mathcal{H}_{\rho}^{\beta}$-measurable. The corresponding Hausdorff dimension of $E$ is defined by

$$
\operatorname{dim}_{\mathcal{H}}^{\rho} E=\inf \left\{\beta>0: \mathcal{H}_{\rho}^{\beta}(E)=0\right\} .
$$

In some special cases, Hausdorff measure and dimension of this type have been applied by Kaufman (1972), Hawkes (1978), Taylor and Watson (1985), and Testard (1986) to study the hitting probability of space-time processes of Brownian motion and other processes.

Note that the metric space $\left(\mathbb{R}^{N}, \rho\right)$ is complete and separable. Hence the following generalized Frostman's lemma is a consequence of Theorem 8.17 in Mattila (1995) and a remark on page 117 of the same reference. It can also be proved by using a result of Assouad (1977) [cf. Kahane (1985, p. 137)] on the quasi-helix and the classical Frostman's lemma; see Testard (1986, p. 4) for a special case.

Lemma 6.10 For any Borel set $E \subseteq \mathbb{R}^{N}, \mathcal{H}_{\rho}^{\beta}(E)>0$ if and only if there exist a Borel probability measure on $E$ and a positive constant $c$ such that $\mu\left(B_{\rho}(x, r)\right) \leq c r^{\beta}$ for all $x \in \mathbb{R}^{N}$ and $r>0$. 
We can now prove the following extension of the Hausdorff dimension result for the range of $X$ in Theorem 6.1 .

Theorem 6.11 Suppose the conditions of Theorem 6.9 hold. Then for every Borel set $E \subseteq[0,1]^{N}$,

$$
\operatorname{dim}_{\mathcal{H}} X(E)=\min \left\{d ; \operatorname{dim}_{\mathcal{H}}^{\rho} E\right\} \quad \text { a.s. }
$$

Proof Since the idea for proving (6.47) is quite standard, we only give a sketch of it. For any $\gamma>\operatorname{dim}_{\mathcal{H}}^{\rho} E$, there is a covering $\left\{B_{\rho}\left(r_{n}\right), n \geq 1\right\}$ of $E$ such that $\sum_{n=1}^{\infty}\left(2 r_{n}\right)^{\gamma} \leq 1$. Note that $X(E) \subseteq \cup_{n=1}^{\infty} X\left(B_{\rho}\left(r_{n}\right)\right)$ and the uniform modulus of continuity of $X$ implies that the diameter of $X\left(B_{\rho}\left(r_{n}\right)\right)$ is at most $c r_{n}^{1-\delta}$, where $\delta \in(0,1)$ is a constant. We can show that $\operatorname{dim}_{\mathcal{H}} X(E) \leq \gamma /(1-\delta)$ almost surely. The desired upper bound follows from the arbitrariness of $\gamma$ and $\delta$.

To prove the lower bound, let $\gamma \in\left(0, \min \left\{d ; \operatorname{dim}_{\mathcal{H}}^{\rho} E\right\}\right)$ be fixed. Then by using the generalized Frostman's lemma [Lemma 6.10] one can show that there exists a probability measure $\mu$ on $E$ such that

$$
\iint \frac{1}{\rho(s, t)^{\gamma}} \mu(d s) \mu(d t)<\infty .
$$

This and Condition (C1) immediately imply

$$
\mathbb{E} \iint \frac{\mu(d s) \mu(d t)}{|X(s)-X(t)|^{\gamma}}<\infty
$$

Hence $\operatorname{dim}_{\mathcal{H}} X(E) \geq \min \left\{d ; \operatorname{dim}_{\mathcal{H}}^{\rho} E\right\}$ almost surely.

Combining Theorems 6.9 and 6.11, the invariance properties of $\operatorname{dim}_{\mathcal{H}}^{\rho} E$ and $s(H, E)$, we can derive the following alternative expression for $s(H, E)$. Of course, this can also be proved directly by using measure-theoretic methods.

Corollary 6.12 For every Borel set $E \subseteq \mathbb{R}^{N}$, we have $\operatorname{dim}_{\mathcal{H}}^{\rho} E=s(H, E)$.

As in the case of fractional Brownian sheets considered by Wu and Xiao (2007), the image $X(E)$ has rich Fourier analytic and topological properties. For example, by modifying the proofs in Wu and Xiao (2007), one can prove that if $X$ is a Gaussian random field with stationary increments and spectral density satisfying (3.2) then $X(E)$ is a Salem set [see Kahane (1985) or Mattila (1995) for definition] whenever $\operatorname{dim}_{\mathcal{H}}^{\rho} E \leq d$, and $X(E)$ has interior points whenever $\operatorname{dim}_{\mathcal{H}}^{\rho} E>d$ [It is an exercise to work out the details].

Finally, we consider the special case when $H=\langle\alpha\rangle$. Theorem 6.11 implies that for every Borel set $E \subseteq[0,1]^{N}$,

$$
\operatorname{dim}_{\mathcal{H}} X(E)=\min \left\{d, \frac{1}{\alpha} \operatorname{dim}_{\mathcal{H}} E\right\} \quad \text { a.s. }
$$

The following theorem gives us a uniform version of (6.50).

Theorem 6.13 Let $X=\left\{X(t), t \in \mathbb{R}^{N}\right\}$ be as in Theorem 6.11 with $H=\langle\alpha\rangle$. If $N \leq \alpha d$ and $X$ satisfies either Condition (C3) or $\left(C 3^{\prime}\right)$, then with probability 1

$$
\operatorname{dim}_{\mathcal{H}} X(E)=\frac{1}{\alpha} \operatorname{dim}_{\mathcal{H}} E \text { for all Borel sets } E \subseteq I
$$

and

$$
\operatorname{dim}_{\mathcal{P}} X(E)=\frac{1}{\alpha} \operatorname{dim}_{\mathcal{P}} E \text { for all Borel sets } E \subseteq I .
$$


The proof of Theorem 6.13 is reminiscent to those in Monrad and Pitt (1987), Khoshnevisan, $\mathrm{Wu}$ and Xiao (2006) and $\mathrm{Wu}$ and Xiao (2007). The key step is to apply Condition (C3) or (C3') to prove the following lemma. For simplicity, assume $I=[0,1]^{N}$.

Lemma 6.14 Suppose the assumptions of Theorem 6.13 hold, and let $\delta>0$ and $0<2 \alpha-\delta<\beta<$ $2 \alpha$ be given constants. Then with probability 1 , for all integers $n$ large enough, there do not exist more than $2^{\text {ndd }}$ distinct points of the form $t^{j}=4^{-n} k^{j}$, where $k^{j} \in\left\{1,2, \ldots, 4^{n}\right\}^{N}$, such that

$$
\left|X\left(t^{i}\right)-X\left(t^{j}\right)\right|<3 \cdot 2^{-n \beta} \quad \text { for } i \neq j
$$

Proof A proof of Lemma 6.14 under Condition (C3) is given in Wu and Xiao (2007) [see also Khoshnevisan, $\mathrm{Wu}$ and Xiao (2006)]. The proof under $\left(\mathrm{C} 3^{\prime}\right)$ is similar and is left to the reader as an exercise.

Both (6.50) and Theorem 6.13 imply that sometimes one can determine the packing dimension of the image $X(E)$ by the packing dimension of $E$. However, it follows from the results in Talagrand and Xiao (1996) that the conclusion is false if $N>\alpha d$. The method in Xiao (1997c) shows that if $X=\left\{X(t), t \in \mathbb{R}^{N}\right\}$ is a Gaussian random field satisfying (C1) with $H=\langle\alpha\rangle$ then for every Borel set $E \subseteq I$,

$$
\operatorname{dim}_{\mathcal{P}} X(E)=\frac{1}{\alpha} \operatorname{Dim}_{\alpha d} E \quad \text { a.s. }
$$

where $\operatorname{Dim}_{s} E$ is the packing dimension profile of $E$ defined by Falconer and Howroyd (1997). However, the analogous problem for general anisotropic Gaussian random fields has not been settled.

\section{$7 \quad$ Hausdorff dimension of the level sets and hitting probabilities}

Under Conditions (C1) and (C2), we can study fractal properties of the level set $L_{x}=\{t \in I$ : $X(t)=x\}\left(x \in \mathbb{R}^{d}\right)$ and the hitting probabilities of Gaussian random field $X$.

The following result determines the Hausdorff and packing dimensions of the level set.

Theorem 7.1 Let $X=\left\{X(t), t \in \mathbb{R}^{N}\right\}$ be an $(N, d)$-Gaussian random field satisfying Conditions (C1) and (C2) on $I=[\varepsilon, 1]^{N}$.

(i) If $\sum_{j=1}^{N} \frac{1}{H_{j}}<d$, then for every $x \in \mathbb{R}^{d}, L_{x}=\emptyset$ a.s.

(ii) If $\sum_{j=1}^{N} \frac{1}{H_{j}}>d$, then for every $x \in \mathbb{R}^{d}$,

$$
\begin{aligned}
\operatorname{dim}_{\mathcal{H}} L_{x} & =\operatorname{dim}_{\mathcal{P}} L_{x} \\
& =\min \left\{\sum_{j=1}^{k} \frac{H_{k}}{H_{j}}+N-k-H_{k} d, 1 \leq k \leq N\right\} \\
& =\sum_{j=1}^{k} \frac{H_{k}}{H_{j}}+N-k-H_{k} d, \quad \text { if } \sum_{j=1}^{k-1} \frac{1}{H_{j}} \leq d<\sum_{j=1}^{k} \frac{1}{H_{j}}
\end{aligned}
$$

holds with positive probability. 
Remark 7.2 In the critical case when $\sum_{j=1}^{N} \frac{1}{H_{j}}=d$, it is believed that $L_{x}=\emptyset$ a.s. In the Brownian sheet case, this was proved by Orey and Pruitt (1973, Theorem 3.4). It also follows from a potential theoretic result of Khoshnevisan and Shi (1999). If $X$ is a fractional Brownian motion of index $\alpha \in(0,1)$, then an argument of Talagrand (1998) can be modified to show $L_{x}=\emptyset$ a.s. However, the problem whether $L_{x}=\emptyset$ a.s. for more general Gaussian random fields remains open. A proof would require Condition (C3) or (C3') and some extra conditions on the function $\mathbb{E}\left(X_{1}(t)-X_{1}(s)\right)^{2}$.

Proof of Theorem 7.1. Similar to the proof of Theorem 5 in Ayache and Xiao (2005), we divide the proof of Theorem 7.1 into two steps. In Step one, we prove (i) and the upper bound for $\operatorname{dim}_{\mathcal{p}} L_{x}$ in (7.1); and in Step two we prove the lower bound for $\operatorname{dim}_{\mathcal{H}} L_{x}$ by constructing a random measure on $L_{x}$ and using a capacity argument. Moreover, the last equality in (7.1) follows from Lemma 6.2.

First we prove

$$
\overline{\operatorname{dim}}_{\mathcal{B}} L_{x} \leq \min \left\{\sum_{j=1}^{k} \frac{H_{k}}{H_{j}}+N-k-H_{k} d, 1 \leq k \leq N\right\} \quad \text { a.s. }
$$

and $L_{x}=\emptyset$ a.s. whenever the right hand side of (7.2) is negative. It can be verified that the latter is equivalent to $\sum_{j=1}^{N} \frac{1}{H_{j}}<d$.

For an integer $n \geq 1$, divide the interval $[\varepsilon, 1]^{N}$ into $m_{n}$ sub-rectangles $R_{n, \ell}$ of side lengths $n^{-1 / H_{j}}(j=1, \cdots, N)$. Then $m_{n} \leq c n^{\sum_{j=1}^{N} H_{j}^{-1}}$. Let $0<\delta<1$ be fixed and let $\tau_{n, \ell}$ be the lower-left vertex of $R_{n, \ell}$. Then

$$
\begin{aligned}
\mathbb{P}\left\{x \in X\left(R_{n, \ell}\right)\right\} \leq & \mathbb{P}\left\{\max _{s, t \in R_{n, \ell}}|X(s)-X(t)| \leq n^{-(1-\delta)} ; x \in X\left(R_{n, \ell}\right)\right\} \\
& +\mathbb{P}\left\{\max _{s, t \in R_{n, \ell}}|X(s)-X(t)|>n^{-(1-\delta)}\right\} \\
\leq & \mathbb{P}\left\{\left|X\left(\tau_{n, \ell}\right)-x\right| \leq n^{-(1-\delta)}\right\}+e^{-c n^{2 \delta}} \\
\leq & c n^{-(1-\delta) d} .
\end{aligned}
$$

In the above we have applied Lemma 2.1 in Talagrand (1995) to get the second inequality. If $\sum_{j=1}^{N} \frac{1}{H_{j}}<d$, we choose $\delta>0$ such that $(1-\delta) d>\sum_{j=1}^{N} \frac{1}{H_{j}}$. Let $N_{n}$ be the number of rectangles $R_{n, \ell}$ such that $x \in X\left(R_{n, \ell}\right)$. It follows from (7.3) that

$$
\mathbb{E}\left(N_{n}\right) \leq c n^{\sum_{j=1}^{N} H_{j}^{-1}} n^{-(1-\delta) d} \rightarrow 0 \quad \text { as } n \rightarrow \infty .
$$

Since the random variables $N_{n}$ are integer-valued, (7.4) and Fatou's lemma imply that a.s. $N_{n}=0$ for infinitely many integers $n \geq 1$. Therefore $L_{x}=\emptyset$ almost surely.

Now we assume $\sum_{j=1}^{N} \frac{1}{H_{j}}>d$ and define a covering $\left\{R_{n, \ell}^{\prime}\right\}$ of $L_{x}$ by $R_{n, \ell}^{\prime}=R_{n, \ell}$ if $x \in X\left(R_{n, \ell}\right)$ and $R_{n, \ell}^{\prime}=\emptyset$ otherwise. We will show that there are $N$ different ways to cover $L_{x}$ by using cubes of the same side-lengths and each of these ways leads to an upper bound for $\overline{\operatorname{dim}}_{\mathcal{B}} L_{x}$.

For every $1 \leq k \leq N$, the rectangle $R_{n, \ell}^{\prime}$ can be covered by $n^{\sum_{j=k+1}^{N}\left(H_{k}^{-1}-H_{j}^{-1}\right)}$ cubes of sidelength $n^{-H_{k}^{-1}}$. Thus we can cover the level set $L_{x}$ by a sequence of cubes of side-length $n^{-H_{k}^{-1}}$. 
Denote the number of such cubes by $M_{n, k}$. Using (7.3) again, we have

$$
\begin{aligned}
\mathbb{E}\left(M_{n, k}\right) & \leq c_{7,1} n^{\sum_{j=1}^{N} H_{j}^{-1}} n^{-(1-\delta) d} \cdot n^{\sum_{j=k+1}^{N}\left(H_{k}^{-1}-H_{j}^{-1}\right)} \\
& =c_{7,1} n^{(N-k) H_{k}^{-1}+\sum_{j=1}^{k} H_{j}^{-1}-(1-\delta) d} .
\end{aligned}
$$

Now let $\eta$ be the constant defined by

$$
\eta=(N-k) H_{k}^{-1}+\sum_{j=1}^{k} H_{j}^{-1}-(1-2 \delta) d .
$$

We consider the sequence of integers $n_{i}=2^{i}(i \geq 1)$. Then by (7.5), the Markov inequality and the Borel-Cantelli lemma we see that almost surely $M_{n_{i}, k} \leq c n_{i}^{\eta}$ for all $i$ large enough. This implies that $\overline{\operatorname{dim}}_{\mathcal{B}} L_{x} \leq H_{k} \eta$ almost surely. Letting $\delta \downarrow 0$ along rational numbers, we have

$$
\overline{\operatorname{dim}}_{\mathcal{B}} L_{x} \leq \sum_{j=1}^{k} \frac{H_{k}}{H_{j}}+N-k-H_{k} d \quad \text { a.s. }
$$

Optimizing (7.6) over $k=1, \ldots, N$ yields (7.2).

To prove the lower bound for $\operatorname{dim}_{\mathcal{H}} L_{x}$ in (7.1), we assume $\sum_{j=1}^{k-1} \frac{1}{H_{j}} \leq d<\sum_{j=1}^{k} \frac{1}{H_{j}}$ for some $1 \leq k \leq N$. Let $\delta>0$ be a small constant such that

$$
\gamma:=\sum_{j=1}^{k} \frac{H_{k}}{H_{j}}+N-k-H_{k}(1+\delta) d>N-k .
$$

This is possible by Lemma 6.2. Note that if we can prove that there is a constant $c_{7,2}>0$, independent of $\delta$, such that

$$
\mathbb{P}\left\{\operatorname{dim}_{\mathcal{H}} L_{x} \geq \gamma\right\} \geq c_{7,2},
$$

then the lower bound in (7.1) will follow by letting $\delta \downarrow 0$.

Our proof of (7.8) is based on the capacity argument due to Kahane [see Kahane (1985)]. Similar methods have been used by Adler (1981), Testard (1986), Xiao (1995) to certain Gaussian and stable random fields.

Let $\mathcal{M}_{\gamma}^{+}$be the space of all non-negative measures on $\mathbb{R}^{N}$ with finite $\gamma$-energy. It is known [cf. Adler (1981)] that $\mathcal{M}_{\gamma}^{+}$is a complete metric space under the metric

$$
\|\mu\|_{\gamma}=\int_{\mathbb{R}^{N}} \int_{\mathbb{R}^{N}} \frac{\mu(d t) \mu(d s)}{|t-s|^{\gamma}}
$$

We define a sequence of random positive measures $\mu_{n}:=\mu_{n}(x, \bullet)$ on the Borel sets $C$ of $[\varepsilon, 1]^{N}$ by

$$
\begin{aligned}
\mu_{n}(C) & =\int_{C}(2 \pi n)^{d / 2} \exp \left(-\frac{n|X(t)-x|^{2}}{2}\right) d t \\
& =\int_{C} \int_{\mathbb{R}^{d}} \exp \left(-\frac{|\xi|^{2}}{2 n}+i\langle\xi, X(t)-x\rangle\right) d \xi d t
\end{aligned}
$$


It follows from Kahane $(1985$, p. 206) or Testard $(1986$, p.17) that if there exist positive and finite constants $c_{7,3}, c_{7,4}$ and $c_{7,5}$ such that

$$
\begin{aligned}
& \mathbb{E}\left(\left\|\mu_{n}\right\|\right) \geq c_{7,3}, \quad \mathbb{E}\left(\left\|\mu_{n}\right\|^{2}\right) \leq c_{7,4}, \\
& \mathbb{E}\left(\left\|\mu_{n}\right\|_{\gamma}\right) \leq c_{7,5},
\end{aligned}
$$

where $\left\|\mu_{n}\right\|=\mu_{n}\left([\varepsilon, 1]^{N}\right)$ denotes the total mass of $\mu_{n}$, then there is a subsequence of $\left\{\mu_{n}\right\}$, say $\left\{\mu_{n_{k}}\right\}$, such that $\mu_{n_{k}} \rightarrow \mu$ in $\mathcal{M}_{\gamma}^{+}$and $\mu$ is strictly positive with probability $\geq c_{7,3}^{2} /\left(2 c_{7,4}\right)$. In this case, it follows from (7.10) that $\mu$ has its support in $L_{x}$ almost surely. Moreover, (7.12) and the monotone convergence theorem together imply that the $\gamma$-energy of $\mu$ is finite. Hence Frostman's theorem yields (7.8) with $c_{7,2}=c_{7,3}^{2} /\left(2 c_{7,4}\right)$.

It remains to verify (7.11) and (7.12). By Fubini's theorem we have

$$
\begin{aligned}
\mathbb{E}\left(\left\|\mu_{n}\right\|\right) & =\int_{[\varepsilon, 1]^{N}} \int_{\mathbb{R}^{d}} e^{-i\langle\xi, x\rangle} \exp \left(-\frac{|\xi|^{2}}{2 n}\right) \mathbb{E} \exp (i\langle\xi, X(t)\rangle) d \xi d t \\
& =\int_{[\varepsilon, 1]^{N}} \int_{\mathbb{R}^{d}} e^{-i\langle\xi, x\rangle} \exp \left(-\frac{1}{2}\left(n^{-1}+\sigma^{2}(t)\right)|\xi|^{2}\right) d \xi d t \\
& =\int_{[\varepsilon, 1]^{N}}\left(\frac{2 \pi}{n^{-1}+\sigma^{2}(t)}\right)^{d / 2} \exp \left(-\frac{|x|^{2}}{2\left(n^{-1}+\sigma^{2}(t)\right)}\right) d t \\
& \geq \int_{[\varepsilon, 1]^{N}}\left(\frac{2 \pi}{1+\sigma^{2}(t)}\right)^{d / 2} \exp \left(-\frac{|x|^{2}}{2 \sigma^{2}(t)}\right) d t:=c_{7,3} .
\end{aligned}
$$

Denote by $I_{2 d}$ the identity matrix of order $2 d$ and $\operatorname{Cov}(X(s), X(t))$ the covariance matrix of the random vector $(X(s), X(t))$. Let $\Gamma=n^{-1} I_{2 d}+\operatorname{Cov}(X(s), X(t))$ and $(\xi, \eta)^{\prime}$ be the transpose of the row vector $(\xi, \eta)$. Then

$$
\begin{aligned}
\mathbb{E}\left(\left\|\mu_{n}\right\|^{2}\right) & =\int_{[\varepsilon, 1]^{N}} \int_{[\varepsilon, 1]^{N}} \int_{\mathbb{R}^{d}} \int_{\mathbb{R}^{d}} e^{-i\langle\xi+\eta, x\rangle} \exp \left(-\frac{1}{2}(\xi, \eta) \Gamma(\xi, \eta)^{\prime}\right) d \xi d \eta d s d t \\
& =\int_{[\varepsilon, 1]^{N}} \int_{[\varepsilon, 1]^{N}} \frac{(2 \pi)^{d}}{\sqrt{\operatorname{det} \Gamma}} \exp \left(-\frac{1}{2}(x, x) \Gamma^{-1}(x, x)^{\prime}\right) d s d t \\
& \leq \int_{[\varepsilon, 1]^{N}} \int_{[\varepsilon, 1]^{N}} \frac{(2 \pi)^{d}}{\left[\operatorname{det} \operatorname{Cov}\left(X_{0}(s), X_{0}(t)\right)\right]^{d / 2}} d s d t .
\end{aligned}
$$

It follows from Conditions (C1), (C2) and (3.11) that for all $s, t \in[\varepsilon, 1]^{N}$,

$$
\operatorname{det} \operatorname{Cov}\left(X_{0}(s), X_{0}(t)\right) \geq c_{7,6} \sum_{j=1}^{N}\left|s_{j}-t_{j}\right|^{2 H_{j}}
$$

Combining (7.14), (7.15) and applying Lemma 6.3 repeatedly, we obtain

$$
\mathbb{E}\left(\left\|\mu_{n}\right\|^{2}\right) \leq c_{7,7} \int_{[\varepsilon, 1]^{N}} \int_{[\varepsilon, 1]^{N}} \frac{1}{\left[\sum_{j=1}^{N}\left|s_{j}-t_{j}\right|^{2 H_{j}}\right]^{d / 2}} d s d t:=c_{7,4}<\infty .
$$

This is similar to (6.25)-(6.28) in the proof of Theorem 6.1. Thus we have shown (7.11) holds. 
Similar to (7.14), we have

$$
\begin{aligned}
\mathbb{E}\left(\left\|\mu_{n}\right\|_{\gamma}\right) & =\int_{[\varepsilon, 1]^{N}} \int_{[\varepsilon, 1]^{N}} \frac{d s d t}{|s-t|^{\gamma}} \int_{\mathbb{R}^{d}} \int_{\mathbb{R}^{d}} e^{-i\langle\xi+\eta, x\rangle} \exp \left(-\frac{1}{2}(\xi, \eta) \Gamma(\xi, \eta)^{\prime}\right) d \xi d \eta \\
& \leq c_{7,8} \int_{[\varepsilon, 1]^{N}} \int_{[\varepsilon, 1]^{N}} \frac{d s d t}{\left(\sum_{j=1}^{N}\left|s_{j}-t_{j}\right|\right)^{\gamma}\left(\sum_{j=1}^{N}\left|s_{j}-t_{j}\right|^{2 H_{j}}\right)^{d / 2}} \\
& \leq c_{7,9} \int_{0}^{1} d t_{N} \cdots \int_{0}^{1} \frac{d t_{1}}{\left(\sum_{j=1}^{N} t_{j}^{H_{j}}\right)^{d}\left(\sum_{j=1}^{N} t_{j}\right)^{\gamma}},
\end{aligned}
$$

where the two inequalities follow from (7.15) and a change of variables. Note that the last integral in (7.17) is similar to (6.32) and can be estimated by using Lemma 6.4 in the same way as in the proof of $(6.33)-(6.36)$. Moreover, we can take $\delta$ small enough, say $\delta<\delta_{0}$, so that the $\gamma$ defined in (7.7) is bounded away from $N-k$ and $N-k+1$. This implies that $\mathbb{E}\left(\left\|\mu_{n}\right\|_{\gamma}\right) \leq c_{7,9}$ which is independent of $\delta$. This proves (7.12) and hence Theorem 7.1.

In light of Theorem 7.1, it is of interest to further study the following question about fractal properties of the level sets.

Question 7.3 Determine the exact Hausdorff and packing measure functions for the level set $L_{x}$.

Questions 7.3 is closely related to the regularity properties such as the laws of the iterated logarithm of the local times of $X$. The latter will be considered in Section 8. When $X$ is an $(N, d)$ fractional Brownian motion with index $\alpha$, Xiao (1997b) proved that $\varphi_{7}(r)=r^{N-\alpha d}(\log \log 1 / r)^{\alpha d / N}$ is an exact Hausdorff measure function for $L_{x}$. In Theorem 8.11 we give a partial result [i.e., lower bound] for the exact Hausdorff measure of the level set $L_{x}$. It seems that the method in Xiao (1997b) may be modified to determine an exact Hausdorff measure function for the level sets of Gaussian random fields satisfying $(\mathrm{C} 3)$ or $\left(\mathrm{C} 3{ }^{\prime}\right)$.

So far no exact packing measure results have been proved for the level sets of fractional Brownian motion or the Brownian sheet. These problems are related to the liminf behavior of the local times of $X$ which are more difficult to study.

More general than level sets, one can consider the following questions:

Question 7.4 Given a Borel set $F \subseteq \mathbb{R}^{d}$, when do we have $\mathbb{P}\{X(I) \cap F \neq \varnothing\}>0$ ?

Question 7.5 If $\mathbb{P}\{X(I) \cap F \neq \varnothing\}>0$, what are the Hausdorff and packing dimensions of the inverse image $X^{-1}(F) \cap I$ ?

Question 7.4 is an important question in potential theory of random fields. Complete answer has only been known for a few types of random fields with certain Markov structures. We mention that Khoshnevisan and Shi (1999) proved that if $X$ is an $(N, d)$-Brownian sheet, then for every Borel set $F \subseteq \mathbb{R}^{d}$,

$$
\mathbb{P}\{X(I) \cap F \neq \varnothing\}>0 \Longleftrightarrow \mathcal{C}_{d-2 N}(F)>0 .
$$

Recall that $\mathcal{C}_{\alpha}$ denotes the Bessel-Riesz capacity of order $\alpha$. Dalang and Nualart (2004) have recently extended the methods of Khoshnevisan and Shi (1999) and proved similar results for the solution of a system of $d$ nonlinear hyperbolic stochastic partial differential equations with two variables. In this context, we also mention that Khoshnevisan and Xiao (2002, 2003, 2005, 2007b) and Khoshnevisan, Xiao and Zhong (2003) have established systematic potential theoretical 
results for additive Lévy processes in $\mathbb{R}^{d}$. The arguments in the aforementioned work rely on the multiparameter martingale theory; we refer to Khoshnevisan (2002) for more information on the latter as well as on potential theory of random fields.

For random fields with general dependence structures, it is more difficult to solve Question 7.4 completely. Instead, one can look for sufficient conditions and necessary conditions on $F$ so that $\mathbb{P}\{X(I) \cap F \neq \varnothing\}>0$. For example, when $X$ is an $(N, d)$-fractional Brownian motion, Testard (1986) and Xiao (1999) proved the following results:

$$
\mathcal{C}_{d-N / \alpha}(F)>0 \Rightarrow \mathbb{P}\{X(I) \cap F \neq \varnothing\}>0 \Rightarrow \mathcal{H}^{d-N / \alpha}(F)>0 .
$$

Similar results for the solution to a non-linear stochastic heat equation with multiplicative noise have been proved recently by Dalang, Khoshnevisan and Nualart (2007a).

The following theorem is an analogue of (7.19) for all Gaussian random fields $X$ satisfying Conditions (C1) and (C2).

Theorem 7.6 Assume that an $(N, d)$-Gaussian random field $X=\left\{X(t), t \in \mathbb{R}^{N}\right\}$ satisfies Conditions (C1) and (C2) on $I$ and $d>Q$. Then for every Borel set $F \subseteq \mathbb{R}^{d}$,

$$
c_{7,10} \mathcal{C}_{d-Q}(F) \leq \mathbb{P}\{X(I) \cap F \neq \varnothing\} \leq c_{7,11} \mathcal{H}_{d-Q}(F),
$$

where $Q=\sum_{j=1}^{N} \frac{1}{H_{j}}, c_{7,10}$ and $c_{7,11}$ are positive constants depending on $I, F$ and $H$ only.

Remark 7.7 When $d<Q$, Theorem 7.1 tells us that $X$ hits points, hence (7.20) holds automatically. When $d=Q$, our proof below shows that the lower bound in (7.20) remains to be true and $\mathcal{C}_{0}$ means the logarithmic capacity [see (6.1)]. This can be seen by estimating the integral in (7.26) when $d=Q$. However, if $\mathcal{C}_{0}(F)>0$, then the upper bound in (7.20) becomes $\infty$, thus not informative.

Proof of Theorem 7.6 The lower bound in (7.20) can be proved by using a second moment argument. In fact one can follow the method in Khoshnevisan and Shi (1999) and Dalang and Nualart (2004) to prove the lower bound in (7.20).

In the following, we provide an alternative proof for the lower bound which is similar to that of Theorem 7.1. For any Borel probability measure $\kappa$ on $F$ with $\mathcal{E}_{d-Q}(\kappa)<\infty$ and for all integers $n \geq 1$, we consider a family of random measures $\nu_{n}$ on $I$ defined by

$$
\begin{aligned}
\int_{I} f(t) \nu_{n}(d t) & =\int_{I} \int_{\mathbb{R}^{d}}(2 \pi n)^{d / 2} \exp \left(-n|X(t)-x|^{2}\right) f(t) \kappa(d x) d t \\
& =\int_{I} \int_{\mathbb{R}^{d}} \int_{\mathbb{R}^{d}} \exp \left(-\frac{|\xi|^{2}}{2 n}+i\langle\xi, X(t)-x\rangle\right) f(t) d \xi \kappa(d x) d t,
\end{aligned}
$$

where $f$ is an arbitrary measurable function on $I$. We claim that the following two inequalities hold:

$$
\mathbb{E}\left(\left\|\nu_{n}\right\|\right) \geq c_{7,12}, \quad \mathbb{E}\left(\left\|\nu_{n}\right\|^{2}\right) \leq c_{7,13} \varepsilon_{d-Q}(\kappa),
$$

where the constants $c_{7,12}$ and $c_{7,13}$ are independent of $\kappa$ and $n$.

Since the proof of the first inequality in (7.22) is very similar to (7.13) in the proof of Theorem 7.1 , we only prove the second inequality in (7.22). 
Denote by $I_{2 d}$ the identity matrix of order $2 d$ and $\operatorname{Cov}(X(s), X(t))$ the covariance matrix of the random vector $(X(s), X(t))$. Let $\Gamma_{n}=n^{-1} I_{2 d}+\operatorname{Cov}(X(s), X(t))$ and $(\xi, \eta)^{\prime}$ be the transpose of the row vector $(\xi, \eta)$. Since $\Gamma_{n}$ is positive definite, we have

$$
\begin{aligned}
\mathbb{E}\left(\left\|\nu_{n}\right\|^{2}\right)= & \int_{I} \int_{I} \int_{\mathbb{R}^{2 d}} \int_{\mathbb{R}^{2 d}} e^{-i(\langle\xi, x\rangle+\langle\eta, y\rangle)} \\
& \times \exp \left(-\frac{1}{2}(\xi, \eta) \Gamma_{n}(\xi, \eta)^{\prime}\right) d \xi d \eta \kappa(d x) \kappa(d y) d s d t \\
= & \int_{I} \int_{I} \int_{\mathbb{R}^{2 d}} \frac{(2 \pi)^{d}}{\sqrt{\operatorname{det} \Gamma_{n}}} \exp \left(-\frac{1}{2}(x, y) \Gamma_{n}^{-1}(x, y)^{\prime}\right) \kappa(d x) \kappa(d y) d s d t .
\end{aligned}
$$

By modifying an argument from Testard (1986), we can prove that, under conditions (C1) and (C2), we have

$$
\frac{(2 \pi)^{d}}{\sqrt{\operatorname{det} \Gamma_{n}}} \exp \left(-\frac{1}{2}(x, y) \Gamma_{n}^{-1}(x, y)^{\prime}\right) \leq \frac{c_{7,14}}{\max \left\{\rho^{d}(s, t),|x-y|^{d}\right\}}
$$

for all $s, t \in I$ and $x, y \in \mathbb{R}^{d}$. See Biermé, Laucaux and Xiao (2007) for details. Hence, it follows from (7.23) and (7.24) that

$$
\mathbb{E}\left(\left\|\nu_{n}\right\|^{2}\right) \leq c_{7,14} \int_{I} \int_{I} \int_{\mathbb{R}^{2 d}} \frac{1}{\max \left\{\rho^{d}(s, t),|x-y|^{d}\right\}} \kappa(d x) \kappa(d y) d s d t .
$$

We can verify that for all $x, y \in \mathbb{R}^{d}$,

$$
\int_{I} \int_{I} \frac{d s d t}{\max \left\{\rho^{d}(s, t),|x-y|^{d}\right\}} \leq c_{7,15}|x-y|^{-(d-Q)}
$$

where $c_{7,15}>0$ is a constant. This can be done by breaking the integral in (7.26) over the regions $\{(s, t) \in I \times I: \rho(s, t) \leq|x-y|\}$ and $\{(s, t) \in I \times I: \rho(s, t)>|x-y|\}$, and estimate them separately. It is clear that (7.23), (7.26) and Fubini's theorem imply the second inequality in (7.22).

By using (7.22) and the Paley-Zygmund inequality [cf. Kahane (1985), p.8], one can verify that there is a subsequence of $\left\{\nu_{n}, n \geq 1\right\}$ that converges weakly to a finite measure $\nu$ which is positive with positive probability [depending on $c_{7,12}$ and $c_{7,13}$ only] and $\nu$ also satisfies (7.22). Since $\nu$ is supported on $X^{-1}(F) \cap I$, we use the Paley-Zygmund inequality again to derive

$$
\mathbb{P}\{X(I) \cap F \neq \varnothing\} \geq \mathbb{P}\{\|\nu\|>0\} \geq \frac{[\mathbb{E}(\|\nu\|)]^{2}}{\mathbb{E}\left[\|\nu\|^{2}\right]} \geq \frac{c_{7,16}}{\varepsilon_{d-Q}(\kappa)},
$$

where $c_{7,16}=c_{7,12}^{2} / c_{7,13}$. This implies the lower bound in (7.20).

Our proof of the upper bound in (7.20) relies on the following lemma on the hitting probability of $X$, whose proof will be deferred to the end of this section.

Lemma 7.8 Let $X=\left\{X(t), t \in \mathbb{R}^{N}\right\}$ be an $(N, d)$-Gaussian random field satisfying Conditions (C1) and (C2) on I. Then there exists a constant $c_{7,17}>0$ such that for all $x \in I$ and $y \in \mathbb{R}^{d}$,

$$
\mathbb{P}\left\{\inf _{t \in B_{\rho}(x, r)}|X(t)-y| \leq r\right\} \leq c_{7,17} r^{d}
$$


Now we proceed to prove the upper bound in (7.20) by using a simple covering argument. Choose and fix an arbitrary constant $\gamma>\mathcal{H}_{d-Q}(F)$. By the definition of $\mathcal{H}_{d-Q}(F)$, there is a sequence of balls $\left\{B\left(y_{j}, r_{j}\right), j \geq 1\right\}$ in $\mathbb{R}^{d}$ such that

$$
F \subseteq \bigcup_{j=1}^{\infty} B\left(y_{j}, r_{j}\right) \quad \text { and } \quad \sum_{j=1}^{\infty}\left(2 r_{j}\right)^{d-Q} \leq \gamma .
$$

Clearly we have

$$
\{F \cap X(I) \neq \varnothing\} \subseteq \bigcup_{j=1}^{\infty}\left\{B\left(y_{j}, r_{j}\right) \cap X(I) \neq \varnothing\right\} .
$$

For every $j \geq 1$, we divide the interval $I$ into $c r_{j}^{-Q}$ intervals of side-lengths $r_{j}^{-1 / H_{\ell}}(\ell=1, \ldots, N)$. Hence $I$ can be covered by at most $c r_{j}^{-Q}$ many balls of radius $r_{j}$ in the metric $\rho$. It follows from Lemma 7.8 that

$$
\mathbb{P}\left\{B\left(y_{j}, r_{j}\right) \cap X(I) \neq \varnothing\right\} \leq c r_{j}^{d-Q} .
$$

Combining (7.30) and (7.31) we derive that $\mathbb{P}\{F \cap X(I) \neq \varnothing\} \leq c \gamma$. Since $\gamma>\mathcal{H}_{d-Q}(F)$ is arbitrary, the upper bound in (7.20) follows.

The following are some further remarks and open questions related to Theorem 7.6.

Remark 7.9 For any Borel set $F \subseteq \mathbb{R}^{d}$, Theorem 7.6 provides a sufficient condition and a necessary condition for $\mathbb{P}\left\{X^{-1}(F) \cap I \neq \varnothing\right\}>0$. It is interesting to determine the Hausdorff and packing dimensions of $X^{-1}(F)$ when it is not empty. Recently, Biermé, Lacaux and Xiao (2007) determined the Hausdorff dimension of $X^{-1}(F)$. Namely, they proved that if $\operatorname{dim}_{\mathcal{H}} F>d-\sum_{\ell=1}^{N} \frac{1}{H_{\ell}}$, then

$$
\left\|\operatorname{dim}_{\mathcal{H}}\left(X^{-1}(F) \cap I\right)\right\|_{L^{\infty}(\mathbb{P})}=\min _{1 \leq k \leq N}\left\{\sum_{j=1}^{k} \frac{H_{k}}{H_{j}}+N-k-H_{k}\left(d-\operatorname{dim}_{\mathcal{H}} F\right)\right\} .
$$

Here, for any function $Y: \Omega \rightarrow \mathbb{R}_{+},\|Y\|_{L^{\infty}(\mathbb{P})}$ is defined as

$$
\|Y\|_{L^{\infty}(\mathbb{P})}=\sup \{\theta: Y \geq \theta \text { on an event } E \text { with } \mathbb{P}(E)>0\} .
$$

However, except for the special case of $F=\{x\}$, there have been no results on the packing dimension of $X^{-1}(F)$ for a given Borel set $F \subseteq \mathbb{R}^{d}$.

Remark 7.10 Note that the event on which (7.32) holds depends on $F$. Motivated by the results in Monrad and Pitt (1987), we may ask the following natural question: When $\sum_{\ell=1}^{N} \frac{1}{H_{\ell}}>d$, is it possible to have a single event $\Omega_{1} \subseteq \Omega$ of positive probability such that on $\Omega_{1}$ (7.32) holds for all Borel sets $F \subseteq \mathbb{R}^{d}$ ?

Here are some partial answers. If in addition to Conditions (C1) and (C2), we also assume Condition (C3) or $\left(\mathrm{C} 3^{\prime}\right)$ holds and $H_{1}=H_{2}=\cdots=H_{N}$, then one can modify the proofs in Monrad and Pitt (1987) to show that the answer to the above question is affirmative. In general, it can be proved that, when $\sum_{\ell=1}^{N} \frac{1}{H_{\ell}}>d$, the upper bound in (7.32) holds almost surely for all Borel sets $F \subseteq \mathbb{R}^{d}$. But it is likely that the lower bound may not hold uniformly due to the anisotropy of $X$. 
Remark 7.11 The method for proving Theorem 7.6 may be extended to provide necessary conditions and sufficient conditions for $\mathbb{P}\{X(E) \cap F \neq \varnothing\}>0$, where $E \subseteq(0, \infty)^{N}$ and $F \subseteq \mathbb{R}^{d}$ are arbitrary Borel sets. Some difficulties arise when both $E$ and $F$ are fractal sets. Testard (1986) obtained some partial results for fractional Brownian motion and, for every fixed Borel set $E \subseteq(0, \infty)^{N}$ (or $F \subseteq \mathbb{R}^{d}$ ), Xiao (1999) characterized the "smallest" set $F$ (or $E$ ) such that $\mathbb{P}\{X(E) \cap F \neq \varnothing\}>0$. No such results on anisotropic Gaussian random fields have been proved.

Finally, let us prove Lemma 7.8. There are two ways to prove (7.28). One is to use the argument in the proof of Proposition 4.4 of Dalang, Khoshnevisan and Nualart (2007a) and the other is reminiscent to the proof of Lemma 3.1 in Xiao (1996b). While the former method is slightly simpler, the latter can be adapted to establish hitting probability estimates of the form (7.42) below for anisotropic Gaussian random fields. Hence we will use an argument similar to that in Xiao (1996b).

Proof of Lemma 7.8 For every integer $n \geq 1$, let $\varepsilon_{n}=r \exp \left(-2^{n+1}\right)$ and denote by $N_{n}=$ $N_{\rho}\left(B_{\rho}(x, r), \varepsilon_{n}\right)$ the minimum number of $\rho$-balls of radius $\varepsilon_{n}$ that are needed to cover $B_{\rho}(x, r)$. Note that $N_{n} \leq c \exp \left(Q 2^{n+1}\right)$ [recall that $Q=\sum_{\ell=1}^{N} 1 / H_{\ell}$ ].

Let $\left\{t_{i}^{(n)}, 1 \leq i \leq N_{n}\right\}$ be a set of the centers of open balls with radius $\varepsilon_{n}$ that cover $B_{\rho}(x, r)$. Denote

$$
r_{n}=\beta \varepsilon_{n} 2^{\frac{n+1}{2}},
$$

where $\beta \geq c_{4,10}$ is a constant to be determined later. Here $c_{4,10}$ is the constant in (4.15).

For all integers $n \geq 1,1 \leq j \leq n$ and $1 \leq i \leq N_{n}$, we consider the following events

$$
\begin{aligned}
& A_{i}^{(j)}=\left\{\left|X\left(t_{i}^{(j)}\right)-y\right| \leq r+\sum_{k=j}^{\infty} r_{k}\right\}, \\
& A^{(n)}=\bigcup_{j=1}^{n} \bigcup_{i=1}^{N_{j}} A_{i}^{(j)}=A^{(n-1)} \cup \bigcup_{i=1}^{N_{n}} A_{i}^{(n)} .
\end{aligned}
$$

Then by a chaining argument, the triangle inequality and (4.15), we have

$$
\mathbb{P}\left\{\inf _{t \in B_{\rho}(x, r)}|X(t)-y| \leq r\right\} \leq \lim _{n \rightarrow \infty} \mathbb{P}\left(A^{(n)}\right) .
$$

By (7.33), we have

$$
\mathbb{P}\left(A^{(n)}\right) \leq \mathbb{P}\left(A^{(n-1)}\right)+\mathbb{P}\left(A^{(n)} \backslash A^{(n-1)}\right)
$$

and

$$
\mathbb{P}\left(A^{(n)} \backslash A^{(n-1)}\right) \leq \sum_{i=1}^{N_{n}} \mathbb{P}\left(A_{i}^{(n)} \backslash A_{i^{\prime}}^{(n-1)}\right),
$$

where $i^{\prime}$ is chosen so that $\rho\left(t_{i}^{(n)}, t_{i^{\prime}}^{(n-1)}\right)<\varepsilon_{n-1}$. Note that

$$
\begin{aligned}
& \mathbb{P}\left(A_{i}^{(n)} \backslash A_{i^{\prime}}^{(n-1)}\right) \\
& =\mathbb{P}\left\{\left|X\left(t_{i}^{(n)}\right)-y\right|<r+\sum_{k=n}^{\infty} r_{k},\left|X\left(t_{i^{\prime}}^{(n-1)}\right)-y\right|>r+\sum_{k=n-1}^{\infty} r_{k}\right\} \\
& \leq \mathbb{P}\left\{\left|X\left(t_{i}^{(n)}\right)-y\right|<r+\sum_{k=n}^{\infty} r_{k},\left|X\left(t_{i}^{(n)}\right)-X\left(t_{i^{\prime}}^{(n-1)}\right)\right| \geq r_{n-1}\right\} .
\end{aligned}
$$


By the elementary properties of Gaussian random variables, we can write

$$
\frac{X\left(t_{i}^{(n)}\right)-X\left(t_{i^{\prime}}^{(n-1)}\right)}{\sigma\left(t_{i}^{(n)}, t_{i^{\prime}}^{(n-1)}\right)}=\eta \frac{X\left(t_{i}^{(n)}\right)}{\sigma\left(t_{i}^{(n)}\right)}+\sqrt{1-\eta^{2}} \Xi,
$$

where

$$
\eta=\frac{\mathbb{E}\left[\left(X_{1}\left(t_{i}^{(n)}\right)-X_{1}\left(t_{i^{\prime}}^{(n-1)}\right)\right) X_{1}\left(t_{i}^{(n)}\right)\right]}{\sigma\left(t_{i}^{(n)}, t_{i^{\prime}}^{(n-1)}\right) \sigma\left(t_{i}^{(n)}\right)}
$$

and where $\Xi$ is a centered Gaussian random vector in $\mathbb{R}^{d}$ with the identity matrix as its covariance matrix and, moreover, $\Xi$ is independent of $X\left(t_{i}^{(n)}\right)$.

We observe that

$$
\begin{aligned}
r+\sum_{k=n}^{\infty} r_{k} & \leq r+\sum_{k=0}^{\infty} r_{k} \\
& \leq\left(1+c \int_{0}^{\infty} \exp \left(-\alpha x^{2}\right) d x\right) r:=c_{7,18} r .
\end{aligned}
$$

It follows from Condition (C1) that (7.37) is bounded by

$$
\begin{aligned}
& \mathbb{P}\left\{\left|X\left(t_{i}^{(n)}\right)-y\right| \leq c_{7,18} r,|\Xi| \geq \frac{1}{\sqrt{1-\eta^{2}}}\left[\frac{r_{n-1}}{\rho\left(t_{i}^{(n)}, t_{i^{\prime}}^{(n-1)}\right)}-\eta \frac{X\left(t_{i}^{(n)}\right)}{\sigma\left(t_{i}^{(n)}\right)}\right]\right\} \\
& \leq \mathbb{P}\left\{\left|X\left(t_{i}^{(n)}\right)-y\right| \leq c_{7,18} r,|\Xi| \geq \frac{\beta d}{2} 2^{\frac{n}{2}}\right\} \\
& \quad+\mathbb{P}\left\{\left|X\left(t_{i}^{(n)}\right)-y\right| \leq c_{7,18} r, \eta \frac{\left|X\left(t_{i}^{(n)}\right)\right|}{\sigma\left(t_{i}^{(n)}\right)} \geq \frac{\beta d}{2} 2^{\frac{n}{2}}\right\} \\
& :=I_{1}+I_{2} .
\end{aligned}
$$

By the independence of $\Xi$ and $X\left(t_{i}^{(n)}\right)$, we have

$$
\begin{aligned}
I_{1} & =\mathbb{P}\left\{\left|X\left(t_{i}^{(n)}\right)-y\right| \leq c_{7,18} r\right\} \cdot \mathbb{P}\left\{|\Xi| \geq \frac{\beta d}{2} 2^{\frac{n}{2}}\right\} \\
& \leq c_{7,19} r^{d} \exp \left(-\frac{(\beta d)^{2}}{16} 2^{n}\right) .
\end{aligned}
$$

On the other hand,

$$
\begin{aligned}
I_{2} & \leq \int_{\left\{|u-y| \leq c_{7,18} r,|u| \geq \frac{\beta d}{2} 2^{n / 2} \sigma\left(t_{i}^{(n)}\right)\right\}}\left(\frac{1}{2 \pi}\right)^{\frac{d}{2}} \frac{1}{\sigma^{d}\left(t_{i}^{(n)}\right)} \exp \left(-\frac{|u|^{2}}{2 \sigma^{2}\left(t_{i}^{(n)}\right)}\right) d u \\
& \leq c_{7,20} \int_{\left\{|u-y| \leq c_{7,18} r\right\}} \frac{1}{\sigma^{d}\left(t_{i}^{(n)}\right)} \exp \left(-\frac{|u|^{2}}{4 \sigma^{2}\left(t_{i}^{(n)}\right)}\right) d u \cdot \exp \left(-\frac{(\beta d)^{2}}{16} 2^{n}\right) \\
& \leq c_{7,21} r^{d} \exp \left(-\frac{(\beta d)^{2}}{16} 2^{n}\right) .
\end{aligned}
$$


Combining (7.35) through (7.40) and choosing $\beta \geq c_{4,10}$ satisfying $(\beta d)^{2}>32$, we obtain

$$
\begin{aligned}
\mathbb{P}\left(A^{(n)}\right) & \leq \mathbb{P}\left(A^{(n-1)}\right)+c_{7,22} N_{n} r^{d} \exp \left(-\frac{(\beta d)^{2}}{16} 2^{n}\right) \\
& \leq c_{7,23}\left[\sum_{k=0}^{\infty} N_{k} \exp \left(-\frac{(\beta d)^{2}}{16} 2^{k}\right)\right] r^{d} \\
& \leq c_{7,24} r^{d} .
\end{aligned}
$$

Therefore, (7.28) follows from (7.34) and (7.41).

When $X$ is an $(N, d)$-fractional Brownian motion of index $\alpha$, Xiao (1999) proved the following hitting probability result: If $N<\alpha d$, then there exist positive and finite constants $c_{7,25}$ and $c_{7,26}$, depending only on $N, d$ and $\alpha$, such that for any $r>0$ small enough and any $y \in \mathbb{R}^{d}$ with $|y| \geq r$, we have

$$
c_{7,25}\left(\frac{r}{|y|}\right)^{d-\frac{N}{\alpha}} \leq \mathbb{P}\left\{\exists t \in \mathbb{R}^{N} \text { such that }|X(t)-y|<r\right\} \leq c_{7,26}\left(\frac{r}{|y|}\right)^{d-\frac{N}{\alpha}} .
$$

It would be interesting and useful to establish analogous results for all Gaussian random fields satisfying Conditions (C1) and (C2). Such an estimate will be useful in studying the escape rate and exact packing measure of the sample paths of Gaussian random fields; see Xiao (2003) for the special case of fractional Brownian motion.

\section{Local times and their joint continuity}

We start by briefly recalling some aspects of the theory of local times. For excellent surveys on local times of random and deterministic vector fields, we refer to Geman and Horowitz (1980) and Dozzi (2002).

Let $X(t)$ be a Borel vector field on $\mathbb{R}^{N}$ with values in $\mathbb{R}^{d}$. For any Borel set $T \subseteq \mathbb{R}^{N}$, the occupation measure of $X$ on $T$ is defined as the following Borel measure on $\mathbb{R}^{d}$ :

$$
\mu_{T}(\bullet)=\lambda_{N}\{t \in T: X(t) \in \bullet\} .
$$

If $\mu_{T}$ is absolutely continuous with respect to the Lebesgue measure $\lambda_{d}$, we say that $X(t)$ has a local time on $T$, and define its local time, $L(\bullet, T)$, as the Radon-Nikodým derivative of $\mu_{T}$ with respect to $\lambda_{d}$, i.e.,

$$
L(x, T)=\frac{d \mu_{T}}{d \lambda_{d}}(x), \quad \forall x \in \mathbb{R}^{d} .
$$

In the above, $x$ is the so-called space variable, and $T$ is the time variable. Sometimes, we write $L(x, t)$ in place of $L(x,[0, t])$. It is clear that if $X$ has local times on $T$, then for every Borel set $S \subseteq T, L(x, S)$ also exists.

By standard martingale and monotone class arguments, one can deduce that the local times of $X$ have a version, still denoted by $L(x, T)$, such that it is a kernel in the following sense:

(i). For each fixed $S \subseteq T$, the function $x \mapsto L(x, S)$ is Borel measurable in $x \in \mathbb{R}^{d}$. 
(ii). For every $x \in \mathbb{R}^{d}, L(x, \cdot)$ is Borel measure on $\mathcal{B}(T)$, the family of Borel subsets of $T$.

Moreover, $L(x, T)$ satisfies the following occupation density formula: For every Borel set $T \subseteq \mathbb{R}^{N}$ and for every measurable function $f: \mathbb{R}^{d} \rightarrow \mathbb{R}_{+}$,

$$
\int_{T} f(X(t)) d t=\int_{\mathbb{R}^{d}} f(x) L(x, T) d x .
$$

[cf. Geman and Horowitz (1980, Theorems 6.3 and 6.4)]

Suppose we fix a rectangle $T=\prod_{i=1}^{N}\left[a_{i}, a_{i}+h_{i}\right]$ in $\mathcal{A}$. Then, whenever we can choose a version of the local time, still denoted by $L\left(x, \prod_{i=1}^{N}\left[a_{i}, a_{i}+t_{i}\right]\right)$, such that it is a continuous function of $\left(x, t_{1}, \cdots, t_{N}\right) \in \mathbb{R}^{d} \times \prod_{i=1}^{N}\left[0, h_{i}\right], X$ is said to have a jointly continuous local time on $T$. When a local time is jointly continuous, $L(x, \bullet)$ can be extended to be a finite Borel measure supported on the level set

$$
X_{T}^{-1}(x)=\{t \in T: X(t)=x\}
$$

see Adler (1981) for details. In other words, local times often act as a natural measure on the level sets of $X$ for applying the capacity argument. As such, they are useful in studying the various fractal properties of level sets and inverse images of the vector field $X$. In this regard, we refer to Berman (1972), Ehm (1981), Rosen (1984), Monrad and Pitt (1987) and Xiao (1997b).

First we consider the existence of the local times of Gaussian random fields.

Theorem 8.1 Let $X=\left\{X(t), t \in \mathbb{R}^{N}\right\}$ be an $(N, d)$-Gaussian field defined by (2.26) and suppose Condition (C1) is satisfied on $I$. Then $X$ has a local time $L(x, I) \in L^{2}\left(\mathbb{P} \times \lambda_{d}\right)$ if and only if $d<\sum_{j=1}^{N} 1 / H_{j}$. In the latter case, $L(x, I)$ admits the following $L^{2}$ representation:

$$
L(x, I)=(2 \pi)^{-d} \int_{\mathbb{R}^{d}} e^{-i\langle y, x\rangle} \int_{I} e^{i\langle y, X(s)\rangle} d s d y, \quad \forall x \in \mathbb{R}^{d} .
$$

Proof The Fourier transform of the occupation measure $\mu_{I}$ is

$$
\widehat{\mu}_{I}(\xi)=\int_{I} e^{i\langle\xi, X(t)\rangle} d t
$$

By applying Fubini's theorem twice, we have

$$
\mathbb{E} \int_{\mathbb{R}^{d}}|\widehat{\mu}(\xi)|^{2} d \xi=\int_{I} d s \int_{I} d t \int_{\mathbb{R}^{d}} \mathbb{E} \exp (i\langle\xi, X(s)-X(t)\rangle) d \xi
$$

We denote the right hand side of (8.4) by $\mathcal{J}(I)$. It follows from the Plancherel theorem that $X$ has a local time $L(\cdot, I) \in L^{2}\left(\mathbb{P} \times \lambda_{d}\right)$ if and only if $\mathcal{J}(I)<\infty$; see Theorem 21.9 in Geman and Horowitz (1980), or Kahane (1985). Hence it is sufficient to prove

$$
\mathcal{J}(I)<\infty \quad \text { if and only if } \quad d<\sum_{j=1}^{N} 1 / H_{j} .
$$

For this purpose, we use the independence of the coordinate processes of $X$ and Condition $(\mathrm{C} 1)$ to deduce

$$
\mathcal{J}(I)=\int_{I} \int_{I} \frac{d s d t}{\left[\mathbb{E}\left(X_{0}(s)-X_{0}(t)\right)^{2}\right]^{d / 2}} \asymp \int_{I} \int_{I} \frac{d s d t}{\left(\sum_{j=1}^{N}\left|s_{j}-t_{j}\right|^{2 H_{j}}\right)^{d / 2}}
$$


By using Lemma 8.6 below, it is elementary to verify that the last integral in (8.6) is finite if and only if $d<\sum_{j=1}^{N} 1 / H_{j}$. This proves (8.5), and hence Theorem 8.1.

The following result on the joint continuity of the local times is similar to those proved by Xiao and Zhang (2002), Ayache, Wu and Xiao (2006) for fractional Brownian sheets.

Theorem 8.2 Let $X=\left\{X(t), t \in \mathbb{R}^{N}\right\}$ be an ( $\left.N, d\right)$-Gaussian field defined by (2.26) and we assume Conditions (C1) and $\left(C 3^{\prime}\right)$ are satisfied on $I$. If $d<\sum_{j=1}^{N} 1 / H_{j}$, then $X$ has a jointly continuous local time on $I$.

Remark 8.3 The conclusion of Theorem 8.2 can also be proved to hold for all Gaussian random fields satisfying Conditions (C1) and (C3). The proof follows a similar line, but some modifications are needed in order to prove analogous estimates in Lemmas 8.4 and 8.8. This is left to the reader as an exercise.

To prove Theorem 8.2 we will, similar to Ehm (1981), Xiao (1997b), Ayache, Wu and Xiao (2006), first use the Fourier analytic arguments to derive estimates on the moments of the local times [see Lemmas 8.4 and 8.8 below] and then apply a multiparameter version of Kolmogorov continuity theorem [cf. Khoshnevisan (2002)]. It will be clear that Condition (C3') plays an essential role in the proofs of Lemmas 8.4 and 8.8 .

Our starting points is the following identities about the moments of the local time and its increments. It follows from (25.5) and (25.7) in Geman and Horowitz (1980) [see also Pitt (1978)] that for all $x, y \in \mathbb{R}^{d}, T \in \mathcal{A}$ and all integers $n \geq 1$,

$$
\begin{aligned}
& \mathbb{E}\left[L(x, T)^{n}\right]=(2 \pi)^{-n d} \int_{T^{n}} \int_{\mathbb{R}^{n d}} \exp \left(-i \sum_{j=1}^{n}\left\langle u^{j}, x\right\rangle\right) \\
& \times \mathbb{E} \exp \left(i \sum_{j=1}^{n}\left\langle u^{j}, X\left(t^{j}\right)\right\rangle\right) d \bar{u} d \bar{t}
\end{aligned}
$$

and for all even integers $n \geq 2$,

$$
\begin{aligned}
\mathbb{E}\left[(L(x, T)-L(y, T))^{n}\right]=(2 \pi)^{-n d} & \int_{T^{n}} \int_{\mathbb{R}^{n d}} \prod_{j=1}^{n}\left[e^{-i\left\langle u^{j}, x\right\rangle}-e^{-i\left\langle u^{j}, y\right\rangle}\right] \\
& \times \mathbb{E} \exp \left(i \sum_{j=1}^{n}\left\langle u^{j}, X\left(t^{j}\right)\right\rangle\right) d \bar{u} d \bar{t},
\end{aligned}
$$

where $\bar{u}=\left(u^{1}, \ldots, u^{n}\right), \bar{t}=\left(t^{1}, \ldots, t^{n}\right)$, and each $u^{j} \in \mathbb{R}^{d}, t^{j} \in T \subseteq(0, \infty)^{N}$. In the coordinate notation we then write $u^{j}=\left(u_{1}^{j}, \ldots, u_{d}^{j}\right)$.

Lemma 8.4 Suppose the assumptions of Theorem 8.2 hold. Let $\tau \in\{1, \ldots, N\}$ be the integer such that

$$
\sum_{\ell=1}^{\tau-1} \frac{1}{H_{\ell}} \leq d<\sum_{\ell=1}^{\tau} \frac{1}{H_{\ell}},
$$


then there exists a positive and finite constant $c_{8,1}$, depending on $N, d, H$ and $I$ only, such that for all hypercubes $T=[a, a+\langle r\rangle] \subseteq I$ with side-length $r \in(0,1)$, all $x \in \mathbb{R}^{d}$ and all integers $n \geq 1$,

$$
\mathbb{E}\left[L(x, T)^{n}\right] \leq c_{8,1}^{n} n ! r^{n \beta_{\tau}},
$$

where $\beta_{\tau}=\sum_{\ell=1}^{\tau} \frac{H_{\tau}}{H_{\ell}}+N-\tau-H_{\tau} d$.

\section{Remark 8.5}

(i) It is important to note that, when (8.9) holds, $\beta_{\tau}=\sum_{\ell=1}^{\tau} \frac{H_{\tau}}{H_{\ell}}+N-\tau-H_{\tau} d$ is the Hausdorff dimension of the level set $L_{x}$; see Theorem 7.1. Combining (8.10) with the upper density theorem of Rogers and Taylor (1961), one can obtain some information on the exact Hausdorff measure of $L_{x}$ [see Corollary 8.11 below].

(ii) We point out that the upper bound in (8.10) is not sharp, and one may be able to prove the following inequality:

$$
\mathbb{E}\left[L(x, T)^{n}\right] \leq c_{8,2}^{n}(n !)^{N-\beta_{\tau}} r^{n \beta_{\tau}} .
$$

If this is indeed true, then one can conjecture that the function $\varphi_{8}(r)=r^{\beta_{\tau}}(\log \log 1 / r)^{N-\beta_{\tau}}$ is an exact Hausdorff measure function for $L_{x}$.

For proving Lemma 8.4, we will make use of the following elementary lemma [which is stronger than Lemma 6.3].

Lemma 8.6 Let $\alpha, \beta$ and $A$ be positive constants. Then

$$
\int_{0}^{1} \frac{1}{\left(A+t^{\alpha}\right)^{\beta}} d t \asymp \begin{cases}A^{-\left(\beta-\frac{1}{\alpha}\right)} & \text { if } \alpha \beta>1, \\ \log \left(1+A^{-1 / \alpha}\right) & \text { if } \alpha \beta=1 \\ 1 & \text { if } \alpha \beta<1 .\end{cases}
$$

Proof of Lemma 8.4 Since $X_{1}, \cdots, X_{d}$ are independent copies of $X_{0}$, it follows from (8.7) that for all integers $n \geq 1$,

$$
\mathbb{E}\left[L(x, T)^{n}\right] \leq(2 \pi)^{-n d} \int_{T^{n}} \prod_{k=1}^{d}\left\{\int_{\mathbb{R}^{n}} \exp \left[-\frac{1}{2} \operatorname{Var}\left(\sum_{j=1}^{n} u_{j}^{k} X_{0}\left(t^{j}\right)\right)\right] d U_{k}\right\} d \bar{t}
$$

where $U_{k}=\left(u_{k}^{1}, \cdots, u_{k}^{n}\right) \in \mathbb{R}^{n}$. It is clear that in order to bound the integral in $d \bar{t}$ it is sufficient to consider only the integral over $T_{\neq}^{n}=\left\{\bar{t} \in T^{n}: t^{1}, \ldots, t^{n}\right.$ are distinct] [the set of $\bar{t} \in \mathbb{R}^{N n}$ having $t^{i}=t^{j}$ for some $i \neq j$ has $(N n)$-dimensional Lebesgue measure 0$]$. It follows from Lemma 3.4 that for every $\bar{t} \in T_{\neq}^{n}$, the covariance matrix of $X_{0}\left(t^{1}\right), \cdots, X_{0}\left(t^{n}\right)$ is invertible. We denote the inverse matrix by $R\left(t^{1}, \cdots, t^{n}\right)$, and let $\left(Z_{1}, \cdots, Z_{n}\right)$ be the Gaussian vector with mean zero and the covariance matrix $R\left(t^{1}, \cdots, t^{n}\right)$. Then the density function of $\left(Z_{1}, \cdots, Z_{n}\right)$ is given by

$$
(2 \pi)^{-n / 2}\left[\operatorname{det} \operatorname{Cov}\left(X_{0}\left(t^{1}\right), \ldots, X_{0}\left(t^{n}\right)\right)\right]^{1 / 2} \exp \left(-\frac{1}{2} U \operatorname{Cov}\left(X_{0}\left(t^{1}\right), \ldots, X_{0}\left(t^{n}\right)\right) U^{\prime}\right),
$$


where $U=\left(u^{1}, \cdots, u^{n}\right) \in \mathbb{R}^{n}$ and $U^{\prime}$ is the transpose of $U$. Hence for each $1 \leq k \leq d$,

$$
\int_{\mathbb{R}^{n}} \exp \left[-\frac{1}{2} \operatorname{Var}\left(\sum_{j=1}^{n} u_{k}^{j} X_{0}\left(t^{j}\right)\right)\right] d U_{k}=\frac{(2 \pi)^{n / 2}}{\left[\operatorname{det} \operatorname{Cov}\left(X_{0}\left(t^{1}\right), \ldots, X_{0}\left(t^{n}\right)\right)\right]^{1 / 2}} .
$$

Combining (8.13) and (8.15), we derive

$$
\mathbb{E}\left[L(x, T)^{n}\right] \leq(2 \pi)^{-n d / 2} \int_{T^{n}} \frac{1}{\left[\operatorname{det} \operatorname{Cov}\left(X_{0}\left(t^{1}\right), \ldots, X_{0}\left(t^{n}\right)\right)\right]^{d / 2}} d \bar{t} .
$$

It follows from Condition $\left(\mathrm{C} 3^{\prime}\right)$ and (3.11) that

$$
\begin{aligned}
\operatorname{det} \operatorname{Cov}\left(X_{0}\left(t^{1}\right), \ldots, X_{0}\left(t^{n}\right)\right) & =\prod_{j=1}^{n} \operatorname{Var}\left(X_{0}\left(t^{j}\right) \mid X_{0}\left(t^{i}\right), j<i \leq n\right) \\
& \geq c_{8,3}^{n} \prod_{j=1}^{n} \min _{j<i \leq n+1} \rho\left(t^{j}, t^{i}\right)^{2}
\end{aligned}
$$

where $t^{n+1}=0$. This and (8.16) together imply that

$$
\mathbb{E}\left[L(x, T)^{n}\right] \leq c_{8,4}^{n} \int_{T^{n}} \prod_{j=1}^{n} \frac{1}{\left[\min _{j<i \leq n+1} \rho\left(t^{j}, t^{i}\right)\right]^{d}} d \bar{t} .
$$

We will estimate the integral in (8.18) by integrating in the order $d t^{1}, d t^{2}, \ldots, d t^{n}$. Considering first the integral in $d t^{1}$, we have

$$
\int_{T} \frac{1}{\left[\min _{1<i \leq n+1} \rho\left(t^{j}, t^{i}\right)\right]^{d}} d t^{1} \leq \sum_{i=2}^{n+1} \int_{T} \frac{1}{\left[\rho\left(t^{j}, t^{i}\right)\right]^{d}} d t^{1} \leq c n \int_{[0, r]^{N}} \frac{d s_{1} \cdots d s_{N}}{\left[\sum_{k=1}^{N} s_{k}^{H_{k}}\right]^{d}}
$$

where the last inequality follows from a change of variables. Integrating the last integral in the order $d s_{1}, \cdots, d s_{N}$ and applying (8.12) in Lemma 6.3, we can show that, because of (8.9), the last integrand in (8.19) only affects the integral in $d s_{1}, \ldots, d s_{\tau}$ which contributes [up to a constant] the factor $r^{\sum_{\ell=1}^{\tau} \frac{H_{\tau}}{H_{\ell}}-H_{\tau} d}$; and the integral in $d s_{\tau+1}, \ldots, d s_{N}$ contributes the factor $r^{N-\tau}$. In other words, we have

$$
\int_{[0, r]^{N}} \frac{d s_{1} \cdots d s_{N}}{\left[\sum_{k=1}^{N} s_{k}^{H_{k}}\right]^{d}} \leq c_{8,5} r^{\sum_{\ell=1}^{\tau} \frac{H_{\tau}}{H_{\ell}}+N-\tau-H_{\tau} d}
$$

This and (8.19) imply

$$
\int_{T} \frac{1}{\left[\min _{1<i \leq n+1} \rho\left(t^{j}, t^{i}\right)\right]^{d}} d t^{1} \leq c_{8,6} n r^{\sum_{\ell=1}^{\tau} \frac{H_{\tau}}{H_{\ell}}+N-\tau-H_{\tau} d} .
$$

Repeating the same procedure for integrating in $d t^{2}, \ldots, d t^{n}$ in (8.18) yields (8.10). This proves Lemma 8.4. 
Remark 8.7 In the proof of Lemma 8.4, we have assumed $T$ is a hypercube $T=[a, a+\langle r\rangle]$. This is only for convenience and one can consider arbitrary closed intervals $T=\prod_{\ell=1}^{N}\left[a_{\ell}, a_{\ell}+r_{\ell}\right] \subseteq I$. The argument is the same as above, but (8.19) becomes

$$
\int_{T} \frac{1}{\left[\min _{1<i \leq n+1} \rho\left(t^{j}, t^{i}\right)\right]^{d}} d t^{1} \leq c n \int_{\prod_{k=1}^{N}\left[0, r_{k}\right]} \frac{d s_{1} \cdots d s_{N}}{\left[\sum_{k=1}^{N} s_{k}^{H_{k}}\right]^{d}} .
$$

Choose $N$ positive numbers $p_{1}, \ldots, p_{N} \in(0,1)$ defined by

$$
p_{k}=\frac{H_{k}^{-1}}{\sum_{i=1}^{N} H_{i}^{-1}}, \quad(k=1, \ldots, N) .
$$

Then $\sum_{k=1}^{N} p_{k}=1$. By using the following inequality

$$
\sum_{k=1}^{N} s_{k}^{H_{k}} \geq \sum_{k=1}^{N} p_{k} s_{k}^{H_{k}} \geq \prod_{k=1}^{N} s_{k}^{p_{k} H_{k}}, \quad \forall s \in(0, \infty)^{N},
$$

one can verify that

$$
\int_{\prod_{k=1}^{N}\left[0, r_{k}\right]} \frac{d s_{1} \cdots d s_{N}}{\left[\sum_{k=1}^{N} s_{k}^{H_{k}}\right]^{d}} \leq c \lambda_{N}(T)^{1-\nu}
$$

where $\nu=d /\left(\sum_{i=1}^{N} H_{i}^{-1}\right) \in(0,1)$. Combining (8.18), (8.22) and (8.24) we derive that

$$
\mathbb{E}\left[L(x, T)^{n}\right] \leq c_{8,7}^{n} n ! \lambda_{N}(T)^{n(1-\nu)}
$$

holds for every interval $T \subseteq I$. We will apply this inequality in the proof of Theorem 8.2 below.

Lemma 8.4 implies that for all $n \geq 1, L(x, T) \in L^{n}\left(\mathbb{R}^{d}\right)$ a.s. [see Geman and Horowitz (1980, p. 42)]. Our next lemma estimates the moments of the increments of $L(x, T)$ in the space variable $x$.

Lemma 8.8 Assume (8.9) holds for some $\tau \in\{1, \ldots, N\}$. Then there exists a positive and finite constant $c_{8,8}$, depending on $N, d, H$ and $I$ only, such that for all hypercubes $T=[a, a+\langle r\rangle] \subseteq I$, $x, y \in \mathbb{R}^{d}$ with $|x-y| \leq 1$, all even integers $n \geq 1$ and all $\gamma \in(0,1)$ small enough,

$$
\mathbb{E}\left[(L(x, T)-L(y, T))^{n}\right] \leq c_{8,8}^{n}(n !)^{(1+\gamma)}|x-y|^{n \gamma} r^{n\left(\beta_{\tau}-2 H_{\tau} \gamma\right)} .
$$

In order to prove Lemma 8.8, we will make use of the following lemma essentially due to Cuzick and DuPreez (1982) [see also Khoshnevisan and Xiao (2007a)].

Lemma 8.9 Let $Z_{1}, \ldots, Z_{n}$ be mean zero Gaussian variables which are linearly independent, then for any nonnegative function $g: \mathbb{R} \rightarrow \mathbb{R}_{+}$,

$$
\begin{aligned}
\int_{\mathbb{R}^{n}} g\left(v_{1}\right) \exp \left[-\frac{1}{2} \operatorname{Var}\left(\sum_{j=1}^{n} v_{j} Z_{j}\right)\right] d v_{1} \cdots d v_{n} \\
=\frac{(2 \pi)^{(n-1) / 2}}{\left[\operatorname{det} \operatorname{Cov}\left(Z_{1}, \cdots, Z_{n}\right)\right]^{1 / 2}} \int_{-\infty}^{\infty} g\left(\frac{v}{\sigma_{1}}\right) e^{-v^{2} / 2} d v,
\end{aligned}
$$

where $\sigma_{1}^{2}=\operatorname{Var}\left(Z_{1} \mid Z_{2}, \ldots, Z_{n}\right)$ is the conditional variance of $Z_{1}$ given $Z_{2}, \ldots, Z_{n}$. 
Proof of Lemma 8.8 Let $\gamma \in(0,1)$ be a constant whose value will be determined later. Note that by the elementary inequalities

$$
\left|e^{i u}-1\right| \leq 2^{1-\gamma}|u|^{\gamma} \quad \text { for all } u \in \mathbb{R}
$$

and $|u+v|^{\gamma} \leq|u|^{\gamma}+|v|^{\gamma}$, we see that for all $u^{1}, \ldots, u^{n}, x, y \in \mathbb{R}^{d}$,

$$
\prod_{j=1}^{n}\left|e^{-i\left\langle u^{j}, x\right\rangle}-e^{-i\left\langle u^{j}, y\right\rangle}\right| \leq 2^{(1-\gamma) n}|x-y|^{n \gamma} \sum \prod_{j=1}^{\prime} \mid u_{k_{j}}^{j}{ }^{\gamma},
$$

where the summation $\sum^{\prime}$ is taken over all the sequences $\left(k_{1}, \cdots, k_{n}\right) \in\{1, \cdots, d\}^{n}$.

It follows from (8.8) and (8.28) that for every even integer $n \geq 2$,

$$
\begin{aligned}
\mathbb{E}[(L(x, T)- & \left.L(y, T))^{n}\right] \leq(2 \pi)^{-n d} 2^{(1-\gamma) n}|x-y|^{n \gamma} \\
& \times \sum^{\prime} \int_{T^{n}} \int_{\mathbb{R}^{n d}} \prod_{m=1}^{n}\left|u_{k_{m}}^{m}\right|^{\gamma} \mathbb{E} \exp \left(-i \sum_{j=1}^{n}\left\langle u^{j}, X\left(t^{j}\right)\right\rangle\right) d \bar{u} d \bar{t} \\
& \leq c_{8,9}^{n}|x-y|^{n \gamma} \sum^{\prime} \int_{T^{n}} d \bar{t} \\
& \times \prod_{m=1}^{n}\left\{\int_{\mathbb{R}^{n d}}\left|u_{k_{m}}^{m}\right|^{n \gamma} \exp \left[-\frac{1}{2} \operatorname{Var}\left(\sum_{j=1}^{n}\left\langle u^{j}, X\left(t^{j}\right)\right\rangle\right)\right] d \bar{u}\right\}^{1 / n},
\end{aligned}
$$

where the last inequality follows from the generalized Hölder inequality.

Now we fix a vector $\bar{k}=\left(k_{1}, \ldots, k_{n}\right) \in\{1, \cdots, d\}^{n}$ and $n$ distinct points $t^{1}, \ldots, t^{n} \in T$ [the set of such points has full $(n N)$-dimensional Lebesgue measure]. Let $\mathcal{M}=\mathcal{M}(\bar{k}, \bar{t}, \gamma)$ be defined by

$$
\mathcal{M}=\prod_{m=1}^{n}\left\{\int_{\mathbb{R}^{n d}}\left|u_{k_{m}}^{m}\right|^{n \gamma} \exp \left[-\frac{1}{2} \operatorname{Var}\left(\sum_{j=1}^{n}\left\langle u^{j}, X\left(t^{j}\right)\right\rangle\right)\right] d \bar{u}\right\}^{1 / n} .
$$

Note that $X_{\ell}(1 \leq \ell \leq N)$ are independent copies of $X_{0}$. By Lemma 3.4, the random variables $X_{\ell}\left(t^{j}\right)(1 \leq \ell \leq N, 1 \leq j \leq n)$ are linearly independent. Hence Lemma 8.9 gives

$$
\begin{aligned}
\int_{\mathbb{R}^{n d}} & \left|u_{k_{m}}^{m}\right|^{n \gamma} \exp \left[-\frac{1}{2} \operatorname{Var}\left(\sum_{j=1}^{n}\left\langle u^{j}, X\left(t^{j}\right)\right\rangle\right)\right] d \bar{u} \\
& =\frac{(2 \pi)^{(n d-1) / 2}}{\left[\operatorname{det} \operatorname{Cov}\left(X_{0}\left(t^{1}\right), \ldots, X_{0}\left(t^{n}\right)\right)\right]^{d / 2}} \int_{\mathbb{R}}\left(\frac{v}{\sigma_{m}}\right)^{n \gamma} e^{-\frac{v^{2}}{2}} d v \\
& \leq \frac{c_{8,10}^{n}(n !)^{\gamma}}{\left[\operatorname{det} \operatorname{Cov}\left(X_{0}\left(t^{1}\right), \ldots, X_{0}\left(t^{n}\right)\right)\right]^{d / 2}} \frac{1}{\sigma_{m}^{n \gamma}}
\end{aligned}
$$

where $\sigma_{m}^{2}$ is the conditional variance of $X_{k_{m}}\left(t^{m}\right)$ given $X_{i}\left(t^{j}\right)\left(i \neq k_{m}\right.$ or $i=k_{m}$ but $\left.j \neq m\right)$, and the last inequality follows from Stirling's formula.

Combining (8.30) and (8.31) we obtain

$$
\mathcal{M} \leq \frac{c_{8,11}^{n}(n !)^{\gamma}}{\left[\operatorname{det} \operatorname{Cov}\left(X_{0}\left(t^{1}\right), \ldots, X_{0}\left(t^{n}\right)\right)\right]^{d / 2}} \prod_{m=1}^{n} \frac{1}{\sigma_{m}^{\gamma}} .
$$


The second product in (8.32) will be treated as a "perturbation" factor and will be shown to be small when integrated. For this purpose, we use again the independence of the coordinate processes of $X$ and Condition $\left(\mathrm{C} 3^{\prime}\right)$ to derive

$$
\begin{aligned}
\sigma_{m}^{2} & =\operatorname{Var}\left(X_{k_{m}}\left(t^{m}\right) \mid X_{k_{m}}\left(t^{j}\right), j \neq m\right) \\
& \geq c_{8,12}^{2} \min \left\{\rho\left(t^{m}, t^{j}\right)^{2}: j=0 \text { or } j \neq m\right\} .
\end{aligned}
$$

Now we define a permutation $\pi$ of $\{1, \cdots, n\}$ such that

$$
\rho\left(t^{\pi(1)}, 0\right)=\min \left\{\rho\left(t^{i}, 0\right), j=1, \cdots, n\right\}
$$

and if $t^{\pi(m-1)}$ has been defined, we choose $t^{\pi(m)}$ such that

$$
\rho\left(t^{\pi(m)}, t^{\pi(m-1)}\right)=\min \left\{\rho\left(t^{j}, t^{\pi(m-1)}\right), j \in\{1, \cdots, n\} \backslash\{\pi(1), \cdots, \pi(m-1)\}\right\} .
$$

By this definition, we see that for every $m=1, \cdots, n$,

$$
\begin{aligned}
\min & \left\{\rho\left(t^{\pi(m)}, t^{j}\right): j=0 \text { or } j \neq \pi(m)\right\} \\
& =\min \left\{\rho\left(t^{\pi(m)}, t^{\pi(m-1)}\right), \rho\left(t^{\pi(m+1)}, t^{\pi(m)}\right)\right\} .
\end{aligned}
$$

It follows from (8.33), (8.34) and (3.11) that

$$
\begin{aligned}
\prod_{m=1}^{n} \frac{1}{\sigma_{m}^{\gamma}} & \leq c_{8,12}^{-n \gamma} \prod_{m=1}^{n} \frac{1}{\min \left\{\rho\left(t^{\pi(m)}, t^{j}\right)^{\gamma}: j=0 \text { or } j \neq \pi(m)\right\}} \\
& \leq c_{8,13}^{n} \prod_{m=1}^{n} \frac{1}{\min \left\{\rho\left(t^{\pi(m)}, t^{\pi(m-1)}\right)^{\gamma}, \rho\left(t^{\pi(m+1)}, t^{\pi(m)}\right)^{\gamma}\right\}} \\
& \leq c_{8,13}^{n} \prod_{m=1}^{n} \frac{1}{\rho\left(t^{\pi(m)}, t^{\pi(m-1)}\right)^{2 \gamma}} \\
& \leq c_{8,14}^{n} \prod_{m=1}^{n} \frac{1}{\left[\operatorname{Var}\left(X_{0}\left(t^{\pi(m)}\right) \mid X_{0}\left(t^{\pi(i)}\right), i=1, \cdots, m-1\right)\right]^{\gamma}} \\
& =\frac{c_{8,15}^{n}}{\left[\operatorname{det} \operatorname{Cov}\left(X_{0}\left(t^{1}\right), \cdots, X_{0}\left(t^{n}\right)\right)\right]^{\gamma}} .
\end{aligned}
$$

Combining (8.32) and (8.35), we obtain

$$
\mathcal{M} \leq \frac{c_{8,16}^{n}(n !)^{\gamma}}{\left[\operatorname{det} \operatorname{Cov}\left(X_{0}\left(t^{1}\right), \cdots, X_{0}\left(t^{n}\right)\right)\right]^{\frac{d}{2}+\gamma}} \leq \frac{c_{8,17}^{n}(n !)^{\gamma}}{\prod_{j=1}^{n}\left[\min _{j<i \leq n+1} \rho\left(t^{j}, t^{i}\right)\right]^{d+2 \gamma}},
$$

where the last step follows from Condition $\left(\mathrm{C} 3^{\prime}\right)$ and (3.11).

It follows from (8.29), (8.30), (8.32) and (8.36) that

$$
\mathbb{E}\left[(L(x+y, T)-L(x, T))^{n}\right] \leq c_{8,18}^{n}|y|^{n \gamma}(n !)^{\gamma} \int_{T^{n}} \prod_{j=1}^{n} \frac{1}{\left[\min _{j<i \leq n+1} \rho\left(t^{j}, t^{i}\right)\right]^{d+2 \gamma}} d \bar{t}
$$


Note that the last integral in (8.37) is similar to that in (8.18) and can be estimated by integrating in the order $d t^{1}, d t^{2}, \ldots, d t^{n}$. To this end, we take $\gamma \in(0,1)$ small such that

$$
\sum_{\ell=1}^{\tau-1} \frac{1}{H_{\ell}} \leq d+2 \gamma<\sum_{\ell=1}^{\tau} \frac{1}{H_{\ell}}
$$

Then, similar to (8.19)-(8.21), we derive

$$
\int_{T^{n}} \prod_{j=1}^{n} \frac{1}{\left[\min _{j<i \leq n+1} \rho\left(t^{j}, t^{i}\right)\right]^{d+2 \gamma}} d \bar{t} \leq c_{8,19}^{n}(n !)^{1+\gamma} r^{n\left(\sum_{\ell=1}^{\tau} \frac{H_{\tau}}{H_{\ell}}+N-\tau-H_{\tau}(d+2 \gamma)\right)} .
$$

It is now clear that (8.26) follows from (8.37) and (8.39). This proves Lemma 8.8.

Now we are ready to prove Theorem 8.2.

Proof of Theorem 8.2 It follows from Lemma 8.8 and the multiparameter version of Kolmogorov's continuity theorem [cf. Khoshnevisan (2002)] that, for every fixed interval $T \in \mathcal{A}$ such that $T \subseteq I, X$ has almost surely a local time $L(x, T)$ that is continuous for all $x \in \mathbb{R}^{d}$.

To prove the joint continuity, observe that for all $x, y \in \mathbb{R}^{d}, s, t \in I$ and all even integers $n \geq 1$, we have

$$
\begin{aligned}
& \mathbb{E}\left[(L(x,[\varepsilon, s])-L(y,[\varepsilon, t]))^{n}\right] \leq 2^{n-1}\left\{\mathbb{E}\left[(L(x,[\varepsilon, s])-L(x,[\varepsilon, t]))^{n}\right]\right. \\
&\left.+\mathbb{E}\left[(L(x,[\varepsilon, t])-L(y,[\varepsilon, t]))^{n}\right]\right\} .
\end{aligned}
$$

Since the difference $L(x,[\varepsilon, s])-L(x,[\varepsilon, t])$ can be written as a sum of finite number (only depends on $N$ ) of terms of the form $L\left(x, T_{j}\right)$, where each $T_{j} \in \mathcal{A}$ is a closed subinterval of $I$ with at least one edge length $\leq|s-t|$, we can use Lemma 8.4 and Remark 8.7, to bound the first term in (8.40). On the other hand, the second term in (8.40) can be dealt with using Lemma 8.8 as above. Consequently, for some $\gamma \in(0,1)$ small, the right hand side of $(8.40)$ is bounded by $c_{8,20}^{n}(|x-y|+|s-t|)^{n \gamma}$, where $n \geq 2$ is an arbitrary even integer. Therefore the joint continuity of the local times $L(x, t)$ follows again from the multiparameter version of Kolmogorov's continuity theorem. This finishes the proof of Theorem 8.2.

The proof of Theorem 8.2 also provides some information about the modulus of continuity of $L(x, t)$ as a function of $x$ and $t$. It would be interesting to establish sharp local and uniform Hölder conditions for the local time, because such results bear rich information about the irregular properties of the sample functions of $X$; see Berman (1972), Geman and Horowitz (1980), Adler (1981), Xiao (1997b).

By applying Lemma 8.4 and the Borel-Cantelli lemma, one can easily derive the following law of the iterated logarithm for the local time $L(x, \cdot)$ : There exists a positive constant $c_{8,21}$ such that for every $x \in \mathbb{R}^{d}$ and $t \in(0,1)^{N}$,

$$
\limsup _{r \rightarrow 0} \frac{L(x, U(t, r))}{\varphi_{9}(r)} \leq c_{8,21},
$$


where $U(t, r)$ is the open or closed ball [in the Euclidean metric] centered at $t$ with radius $r$ and $\varphi_{9}(r)=r^{\beta_{\tau}} \log \log (1 / r)$. It follows from Fubini's theorem that, with probability one, (8.41) holds for $\lambda_{N}$-almost all $t \in I$. Now we prove a stronger version of this result, which is useful in determining the exact Hausdorff measure of the level set $L_{x}$.

Theorem 8.10 Let $X$ be an $(N, d)$-Gaussian random field defined by (2.26). We assume Conditions (C1) and $\left(C 3^{\prime}\right)$ are satisfied on $I$ and $d<\sum_{j=1}^{N} \frac{1}{H_{j}}$. Let $\tau \in\{1, \ldots, N\}$ be the integer so that (8.9) holds and let $L$ be the jointly continuous local time of $X$. Then, there is a finite constant $c_{8,22}$ such that with probability one,

$$
\limsup _{r \rightarrow 0} \frac{L(x, U(t, r))}{\varphi_{9}(r)} \leq c_{8,22}
$$

holds for $L(x, \cdot)$-almost all $t \in I$.

Proof The proof is similar to that of Theorem 4.1 in Ayache, Wu and Xiao (2006) [see also Xiao (1997b)].

For every integer $k \geq 1$, we consider the random measure $\mu_{k}:=\mu_{k}(x, \bullet)$ on the Borel subsets $C$ of $I$ defined by (7.10) [where the integer $n$ is replaced by $k$ ]. Then, by the occupation density formula (8.1) and the continuity of the function $y \mapsto L(y, C)$, one can verify that almost surely $\mu_{k}(C) \rightarrow L(x, C)$ as $k \rightarrow \infty$ for every Borel set $C \subseteq I$.

For every integer $m \geq 1$, denote $f_{m}(t)=L\left(x, U\left(t, 2^{-m}\right)\right)$. From the proof of Theorem 8.2 we can see that almost surely the functions $f_{m}(t)$ are continuous and bounded. Hence we have almost surely, for all integers $m, n \geq 1$,

$$
\int_{I}\left[f_{m}(t)\right]^{n} L(x, d t)=\lim _{k \rightarrow \infty} \int_{I}\left[f_{m}(t)\right]^{n} \mu_{k}(d t) .
$$

It follows from (8.43), (7.10) and the proof of Proposition 3.1 of Pitt (1978) that for every positive integer $n \geq 1$,

$$
\begin{gathered}
\mathbb{E} \int_{I}\left[f_{m}(t)\right]^{n} L(x, d t)=\left(\frac{1}{2 \pi}\right)^{(n+1) d} \int_{I} \int_{U\left(t, 2^{-m}\right)^{n}} \int_{\mathbb{R}^{(n+1) d}} \exp \left(-i \sum_{j=1}^{n+1}\left\langle x, u^{j}\right\rangle\right) \\
\times \mathbb{E} \exp \left(i \sum_{j=1}^{n+1}\left\langle u^{j}, X\left(s^{j}\right)\right\rangle\right) d \bar{u} d \bar{s},
\end{gathered}
$$

where $\bar{u}=\left(u^{1}, \ldots, u^{n+1}\right) \in \mathbb{R}^{(n+1) d}$ and $\bar{s}=\left(t, s^{1}, \ldots, s^{n}\right)$. Similar to the proof of (8.10) we have that the right hand side of Eq. (8.44) is at most

$$
c_{8,23}^{n} \int_{I} \int_{U\left(t, 2^{-m}\right)^{n}} \frac{d \bar{s}}{\left[\operatorname{det} \operatorname{Cov}\left(X_{0}(t), X_{0}\left(s^{1}\right), \ldots, X_{0}\left(s^{n}\right)\right)\right]^{d / 2}} \leq c_{8,24}^{n} n ! 2^{-m n \beta_{\tau}},
$$

where $c_{8,24}$ is a positive and finite constant depending on $N, d, H$, and $I$ only.

Let $\gamma>0$ be a constant whose value will be determined later. We consider the random set

$$
I_{m}(\omega)=\left\{t \in I: f_{m}(t) \geq \gamma \varphi_{9}\left(2^{-m}\right)\right\} .
$$


Denote by $\nu_{\omega}$ the restriction of the random measure $L(x, \cdot)$ on $I$, that is, $\nu_{\omega}(E)=L(x, E \cap I)$ for every Borel set $E \subseteq \mathbb{R}_{+}^{N}$. Now we take $n=\lfloor\log m\rfloor$, where $\lfloor y\rfloor$ denotes the integer part of $y$. Then by applying (8.45) and Stirling's formula, we have

$$
\begin{aligned}
\mathbb{E}\left[\nu_{\omega}\left(I_{m}\right)\right] & \leq \frac{\mathbb{E} \int_{I}\left[f_{m}(t)\right]^{n} L(x, d t)}{\left[\gamma \varphi_{9}\left(2^{-m}\right)\right]^{n}} \\
& \leq \frac{c_{8,25}^{n} n ! 2^{-m n} \beta_{\tau}}{\gamma^{n} 2^{-m n} \beta_{\tau}(\log m)^{n}} \leq m^{-2},
\end{aligned}
$$

provided $\gamma>0$ is chosen large enough, say, $\gamma \geq c_{8,25} e^{2}:=c_{8,26}$. This implies that

$$
\mathbb{E}\left(\sum_{m=1}^{\infty} \nu_{\omega}\left(I_{m}\right)\right)<\infty .
$$

Therefore, with probability 1 for $\nu_{\omega}$ almost all $t \in I$, we have

$$
\limsup _{m \rightarrow \infty} \frac{L\left(x, U\left(t, 2^{-m}\right)\right)}{\varphi_{9}\left(2^{-m}\right)} \leq c_{8,26} .
$$

Finally, for any $r>0$ small enough, there exists an integer $m$ such that $2^{-m} \leq r<2^{-m+1}$ and (8.47) is applicable. Since the function $\varphi_{9}(r)$ is increasing near $r=0,(8.42)$ follows from (8.47) and a monotonicity argument.

Since $L(x, \cdot)$ is a random Borel measure supported on the level set $L_{x}=\{t \in I: X(t)=x\}$, Theorem 8.10 and the upper density theorem of Rogers and Taylor (1961) imply the following partial result on the exact Hausdorff measure of $L_{x}$.

Corollary 8.11 Assume the conditions of Theorem 8.10 are satisfied. Then there exists a positive constant $c_{8,27}$ such that for every $x \in \mathbb{R}^{d}$, we have

$$
\mathcal{H}^{\varphi_{9}}\left(L_{x}\right) \geq c_{8,27} L(x, I), \quad \text { a.s. }
$$

Proof The proof is left to the reader as an exercise.

We should mention that the estimates in Lemmas 8.4 and 8.8 are not sharp and it would be interesting to improve them. In the rest of this section, we consider the special case when $H=\langle\alpha\rangle$ and $\alpha \in(0,1)$. Many sample path properties of such Gaussian random fields have been studied in Adler (1981), Khoshnevisan (2002), Shieh and Xiao (2006), Xiao (1997b, 2007a). By applying Lemma 2.3 in Xiao (1997b) in place of (8.19), we prove the following sharp estimates.

Lemma 8.12 Let $X$ be an $(N, d)$-Gaussian random field satisfying the conditions (C1) and (C3') with $H=\langle\alpha\rangle$. We assume that $N>\alpha d$. Then there exists a positive and finite constant $c_{8,28}$, depending on $N, d, \alpha$ and $I$ only, such that for all intervals $T=[a, a+\langle r\rangle] \subseteq I$ with edge length $r \in(0,1)$, all $x \in \mathbb{R}^{d}$ and all integers $n \geq 1$,

$$
\mathbb{E}\left[L(x, T)^{n}\right] \leq c_{8,28}^{n}(n !)^{\alpha d / N} r^{n(N-\alpha d)}
$$

and for any $0<\gamma<\min \{1,(N / \alpha-d) / 2\}$, there exists a positive and finite constant $c_{8,29}$ such that

$$
\mathbb{E}\left[(L(x, T)-L(y, T))^{n}\right] \leq c_{8,29}^{n}(n !)^{2 \gamma+\alpha(d+\gamma) / N}|x-y|^{n \gamma} r^{n(N-\alpha(d+\gamma))} .
$$


Note that, for a Gaussian random field $X$ satisfying the assumptions of Lemma 8.12, Eq. (8.49) allows us to improve the results in Theorem 8.10 and Corollary 8.11 by replacing the Hausdorff measure function $\varphi_{9}(r)$ by $\varphi_{7}(r)=r^{N-\alpha d}(\log \log 1 / r)^{\alpha d / N}$.

Moreover, by combining Lemma 8.12 and the argument in Xiao (1997b), one can establish the following sharp local and uniform Hölder conditions for the maximum local time $L^{*}(\bullet)$ of $X$ defined by

$$
L^{*}(T)=\sup _{x \in \mathbb{R}^{d}} L(x, T), \quad \forall T \subseteq I .
$$

Theorem 8.13 Let $X$ be an $(N, d)$-Gaussian random field satisfying the conditions (C1) and (C3') with $H=\langle\alpha\rangle$ and $N>\alpha d$. Then the following statements hold:

(i) There exists a positive and finite constant $c_{8,30}$ such that for every $t \in I$,

$$
\limsup _{r \rightarrow 0} \frac{L^{*}(U(t, r))}{\varphi_{7}(r)} \leq c_{8,30} \quad \text { a.s. }
$$

where $U(t, r)=\{s \in I:|s-t| \leq r\}$.

(ii) There exists a positive and finite constant $c_{8,31}$ such that

$$
\limsup _{r \rightarrow 0} \sup _{t \in I} \frac{L^{*}(U(t, r))}{\varphi_{10}(r)} \leq c_{8,31} \quad \text { a.s. },
$$

where $\varphi_{10}(r)=r^{N-\alpha d}(\log 1 / r)^{\alpha d / N}$.

Proof The proofs of (8.51) and (8.52) are based on Lemma 8.12 and a chaining argument, which is the same as those of Theorems 1.1 and 1.2 in Xiao (1997b) [see also Ehm (1981)]. We leave the details to the reader.

Similar to Xiao (1997b, 2007a), one can apply Lemma 8.12 and Theorem 8.13 to derive further results, such as the Chung-type laws of the iterated logarithm, modulus of nowhere differentiability, tail probability of the local times, for $(N, d)$-Gaussian random fields satisfying the conditions $(\mathrm{C} 1)$ and $\left(\mathrm{C} 3^{\prime}\right)$ with $H=\langle\alpha\rangle$. These are left to the reader as exercises.

The following is our final remark.

Remark 8.14 Both Conditions (C3) and $\left(\mathrm{C} 3^{\prime}\right)$ are useful in studying the existence and regularity of the self-intersection local times of $X$ which, in turn, provide information on the fractal dimensions of the sets of multiple points and multiple times of $X$. When $X$ is an $(N, d)$-fractional Brownian sheet, these problems have been studied by Meerschaert, Wu and Xiao (2008). It is expected that analogous results also hold for Gaussian random fields satisfying Conditions (C1) and (C3').

\section{References}

[1] R. J. Adler (1981), The Geometry of Random Fields. John Wiley \& Sons Ltd., New York.

[2] L. Arnold and P. Imkeller (1996), Stratonovich calculus with spatial parameters and anticipative problems in multiplicative ergodic theory. Stoch. Process. Appl. 62, 19-54. 
[3] A. Ayache (2004), Hausdorff dimension of the graph of the fractional Brownian sheet. Rev. Mat. Iberoamericana, 20, 395-412.

[4] A. Ayache, S. Leger and M. Pontier (2002), Drap Brownien fractionnaire. Potential Theory 17, 31-43.

[5] A. Ayache and M. S. Taqqu (2003), Rate optimality of wavelet series approximations of fractional Brownian sheet. J. Fourier Anal. Appl. 9, 451-471.

[6] A. Ayache, D. Wu and Y. Xiao (2006), Joint continuity of the local times of fractional Brownian sheets. Ann. Inst. H. Poincaré Probab. Statist., (to appear).

[7] A. Ayache and Y. Xiao (2005), Asymptotic properties and Hausdorff dimensions of fractional Brownian sheets. J. Fourier Anal. Appl. 11, 407-439.

[8] R. Balan and D. Kim (2006), The stochastic heat equation driven by a Gaussian noise: Markov property. Preprint.

[9] E. Belinski and W. Linde (2002), Small ball probabilities of fractional Brownian sheets via fractional integration operators. J. Theoret. Probab. 15, 589-612.

[10] A. Benassi, S. Jaffard and D. Roux (1997), Elliptic Gaussian random processes. Rev. Mat. Iberoamericana 13, 19-90.

[11] D. A. Benson, M. M. Meerschaert, B. Baeumer and H. P. Scheffler (2006), Aquifer operator-scaling and the effect on solute mixing and dispersion. Water Resources Research 42, no. 1, W01415 (18 pp.).

[12] C. Berg and G. Forst (1975), Potential Theory on Locally Compact Abelian Groups. Springer-Verlag, New York-Heidelberg.

[13] S. M. Berman (1972), Gaussian sample function: uniform dimension and Hölder conditions nowhere. Nagoya Math. J. 46, 63-86.

[14] S. M. Berman (1973), Local nondeterminism and local times of Gaussian processes. Indiana Univ. Math. J. 23, 69-94.

[15] H. Biermé, A. Estrade, M. M. Meerschaert and Y. Xiao (2008), Sample path properties of operator scaling Gaussian random fields. In Preparation.

[16] H. Biermé, C. Lacaux and Y. Xiao (2007), Hitting probabilities and the Hausdorff dimension of the inverse images of anisotropic Gaussian random fields. Preprint.

[17] H. Biermé, M. M. Meerschaert and H.-P. Scheffler (2007), Operator scaling stable random fields. Stoch. Process. Appl. 117, 312-332.

[18] A. Bonami and A. Estrade (2003), Anisotropic analysis of some Gaussian models. J. Fourier Anal. Appl. 9, 215-236.

[19] J, Cuzick and J. DuPreez (1982), Joint continuity of Gaussian local times. Ann. Probab. 10, 810-817.

[20] R. C. Dalang (1999), Extending martingale measure stochastic integral with applications to spatially homogeneous s.p.d.e.'s. Electron. J. Probab. 4, no. 6, 1-29. Erratum in Electron. J. Probab. 6 (2001), no. $6,1-5$.

[21] R. C. Dalang and N. E. Frangos (1998), The stochastic wave equation in two spatial dimensions. Ann. Probab. 26, 187-212.

[22] R. C. Dalang, D. Khoshnevisan and E. Nualart (2007a), Hitting probabilities for systems of non-linear stochastic heat equations with additive noise. Latin Amer. J. Probab. Statist. (Alea) 3, 231-271.

[23] R. C. Dalang, D. Khoshnevisan and E. Nualart (2007b), Hitting probabilities for the non-linear stochastic heat equation with multiplicative noise. Submitted. 
[24] R. C. Dalang and C. Mueller (2003), Some non-linear s.p.e.e.'s that are second order in time. Electron. J. Probab. 8, No. 1, 1-21.

[25] R. C. Dalang, C. Mueller and L. Zambotti, (2006), Hitting properties of parabolic s.p.d.e.'s with reflection. Ann. Probab. 34, 1423-1450.

[26] R. C. Dalang and E. Nualart (2004), Potential theory for hyperbolic SPDEs. Ann. Probab. 32, 20992148.

[27] R. C. Dalang and M. Sanz-Solé (2005), Regularity of the sample paths of a class of second-order spde's. J. Funct. Anal. 227, 304-337.

[28] R. C. Dalang and M. Sanz-Solé (2007), Hölder regularity of the solution to the stochastic wave equation in dimension three. Memoirs Amer. Math. Soc., (to appear).

[29] S. Davies and P. Hall (1999), Fractal analysis of surface roughness by using spatial data (with discussion). J. Roy. Statist. Soc. Ser. B 61, 3-37.

[30] P. Doukhan, G. Oppenheim and M. S. Taqqu (2003), Theory and Applications of Long-Range Dependence. Birkhaüser, Boston.

[31] M. Dozzi (2002), Occupation density and sample path properties of $N$-parameter processes. Topics in Spatial Stochastic Processes (Martina Franca, 2001), pp. 127-169, Lecture Notes in Math. 1802, Springer, Berlin.

[32] T. Dunker (2000), Estimates for the small ball probabilities of the fractional Brownian sheet. $J$. Theoret. Probab. 13, 357-382.

[33] K. Dzhaparidze and H. van Zanten (2005), Optimality of an explicit series of the fractional Browinan sheet. Statist. Probab. Lett. 71, 295-301.

[34] W. Ehm (1981), Sample function properties of multi-parameter stable processes. Z. Wahrsch. verw Gebiete 56, 195-228.

[35] N. Eisenbaum and D. Khoshnevisan (2002), On the most visited sites of symmetric Markov processes. Stoch. Process. Appl. 101, 241-256.

[36] K. J. Falconer (1990), Fractal Geometry - Mathematical Foundations And Applications. John Wiley \& Sons Ltd., Chichester.

[37] K. J. Falconer and J. D. Howroyd (1997), Packing dimensions for projections and dimension profiles. Math. Proc. Camb. Philo. Soc. 121, 269-286.

[38] T. Funaki (1983), Random motion of strings and related stochastic evolution equations. Nagoya Math. J. 89, 129-193.

[39] T. Funaki, M. Kikuchi and J. Potthoff (2006), Direction-dependent modulus of continuity for random fields. Preprint.

[40] A. M. Garsia, E. Rodemich and H. Jr. Rumsey (1970), A real variable lemma and the continuity of paths of some Gaussian processes. Indiana Univ. Math. J. 20, 565-578.

[41] D. Geman and J. Horowitz (1980), Occupation densities. Ann. Probab. 8, 1-67.

[42] J. Hawkes (1978), Measures of Hausdorff type and stable processes. Mathematika 25, 202-210.

[43] E. Herbin (2006), From $N$ parameter fractional Brownian motions to $N$ parameter multifractional Brownian motions. Rocky Mount. J. Math. 36, 1249-1284.

[44] X. Hu and S. J. Taylor (1994), Fractal properties of products and projections of measures in $\mathbb{R}^{d}$. Math. Proc. Camb. Philos. Soc., 115, 527-544. 
[45] Y. Hu, B. Øksendal and T. Zhang (2000), Stochastic partial differential equations driven by multiparameter fractional white noise. Stochastic Processes, Physics and Geometry: new interplays, II (Leipzig, 1999), 327-337, Amer. Math. Soc., Providence, RI.

[46] J.-P. Kahane (1985), Some Random Series of Functions. 2nd edition, Cambridge University Press, Cambridge.

[47] A. Kamont (1996), On the fractional anisotropic Wiener field. Probab. Math. Statist. 16, 85-98.

[48] R. Kaufman (1972), Measures of Hausdorff-type, and Brownian motion. Mathematika 19, 115-119.

[49] D. Khoshnevisan (2002), Multiparameter Processes: An Introduction to Random Fields. Springer, New York.

[50] D. Khoshnevisan and Z. Shi (1999), Brownian sheet and capacity. Ann. Probab. 27, 1135-1159.

[51] D. Khoshnevisan, D. Wu and Y. Xiao (2006), Sectorial local nondeterminism and the geometry of the Brownian sheet. Electron. J. Probab. 11, 817-843.

[52] D. Khoshnevisan and Y. Xiao (2002), Level sets of additive Lévy processes. Ann. Probab. 30, 62-100.

[53] D. Khoshnevisan and Y. Xiao (2003), Weak unimodality of finite measures, and an application to potential theory of additive Lévy processes. Proc. Amer. Math. Soc. 131, 2611-2616.

[54] D. Khoshnevisan and Y. Xiao (2005), Lévy processes: capacity and Hausdorff dimension. Ann. Probab. 33, 841-878.

[55] D. Khoshnevisan and Y. Xiao (2007a), Images of the Brownian sheet. Trans. Amer. Math. Soc. 359, $3125-3151$.

[56] D. Khoshnevisan and Y. Xiao (2007b), Harmonic analysis of additive Lévy processes. Submitted.

[57] D. Khoshnevisan, Y. Xiao and Y. Zhong (2003), Measuring the range of an additive Lévy process. Ann. Probab. 31, 1097-1141.

[58] N. Kôno (1975), Asymptotoc behavior of sample functions of Gaussian random fields. J. Math. Kyoto Univ. 15, 671-707.

[59] T. Kühn and W. Linde (2002), Optimal series representation of fractional Brownian sheet. Bernoulli 8, 669-696.

[60] S. Kwapień and J. Rosiński (2004), Sample Hölder continuity of stochastic processes and majorizing measures. In: Seminar on Stochastic Analysis, Random Fields and Applications IV, pp. 155-163, Progr. Probab., 58, Birkhäuser, Basel.

[61] M. Ledoux (1996), Isoperimetry and Gaussian analysis. Lecture Notes in Math. 1648, 165-294, Springer-Verlag, Berlin.

[62] W. V. Li and Q.-M. Shao (2001), Gaussian processes: inequalities, small ball probabilities and applications. In Stochastic Processes: Theory and Methods. Handbook of Statistics, 19, (C. R. Rao and D. Shanbhag, editors), pp. 533-597, North-Holland.

[63] M. A. Lifshits (1999), Asymptotic behavior of small ball probabilities. In: Probab. Theory and Math. Statist., Proc. VII International Vilnius Conference (1998). Vilnius, VSP/TEV, pp. 453-468.

[64] W. Linde (2007), Non-determinism of linear operators and lower entropy estimates. Preprint.

[65] D. J. Mason and Y. Xiao (2001), Sample path properties of operator self-similar Gaussian random fields. Teor. Veroyatnost. i Primenen. 46, 94-116.

[66] D. M. Mason and Z. Shi (2001), Small deviations for some multi-parameter Gaussian processes. J. Theoret. Probab. 14, 213-239. 
[67] P. Mattila (1995), Geometry of Sets and Measures in Euclidean Spaces. Cambridge University Press, Cambridge.

[68] M. M. Meerschaert, W. Wang and Y. Xiao (2007), Fernique-type inequalities and moduli of continuity for anisotropic Gaussian random fields. Preprint.

[69] M. M. Meerschaert, D. Wu and Y. Xiao (2008), Self-intersection local times of fractional Brownian sheets. In Preparation.

[70] D. Monrad and L. D. Pitt (1987), Local nondeterminism and Hausdorff dimension. Progress in Probability and Statistics. Seminar on Stochastic Processes 1986, (E, Cinlar, K. L. Chung, R. K. Getoor, Editors), pp. 163-189, Birkhäuser, Boston.

[71] D. Monrad and H. Rootzén (1995), Small values of Gaussian processes and functional laws of the iterated logarithm. Probab. Thory Relat. Fields 101, 173-192.

[72] T. S. Mountford (1989), Uniform dimension results for the Brownian sheet. Ann. Probab. 17, 14541462.

[73] C. Mueller and R. Tribe (2002), Hitting probabilities of a random string. Electron. J. Probab. 7, Paper No. 10, 1-29.

[74] D. Nualart (2006), Stochastic heat equation driven by fractional noise. Preprint.

[75] B. Øksendal and T. Zhang (2000), Multiparameter fractional Brownian motion and quasi-linear stochastic partial differential equations. Stoch. Stoch. Rep. 71, 141-163.

[76] S. Orey and W. E. Pruitt (1973), Sample functions of the $N$-parameter Wiener process. Ann. Probab. 1, 138-163.

[77] L. D. Pitt (1978), Local times for Gaussian vector fields. Indiana Univ. Math. J. 27, 309-330.

[78] L. D. Pitt and R. S. Robeva (2003), On the sharp Markov property for Gaussian random fields and spectral synthesis in spaces of Bessel potentials. Ann. Probab. 31, 1338-1376.

[79] R. S. Robeva and L. D. Pitt (2004), On the equality of sharp and germ $\sigma$-fields for Gaussian processes and fields. Pliska Stud. Math. Bulgar. 16, 183-205.

[80] C. A. Rogers and S. J. Taylor (1961), Functions continuous and singular with respect to a Hausdorff measure. Mathematika 8, 1-31.

[81] J. Rosen (1984), Self-intersections of random fields. Ann. Probab. 12, 108-119.

[82] G. Samorodnitsky and M. S. Taqqu (1994), Stable non-Gaussian Random Processes: Stochastic models with infinite variance. Chapman \& Hall, New York.

[83] N.-R. Shieh and Y. Xiao (2006), Images of Gaussian random fields: Salem sets and interior points. Studia Math. 176, 37-60.

[84] M. Talagrand (1993), New Gaussian estimates for enlarged balls. Geometric Funct. Anal. 3, 502-526.

[85] M. Talagrand (1995), Hausdorff measure of trajectories of multiparameter fractional Brownian motion. Ann. Probab. 23, 767-775.

[86] M. Talagrand (1998), Multiple points of trajectories of multiparameter fractional Brownian motion. Probab. Theory Relat. Fields 112, 545-563.

[87] M. Talagrand and Y. Xiao (1996), Fractional Brownian motion and packing dimension. J. Theoret. Probab. 9, 579-593.

[88] S. J. Taylor (1986), The measure theory of random fractals. Math. Proc. Camb. Philos. Soc. 100, 383-406. 
[89] S. J. Taylor and C. Tricot (1985), Packing measure and its evaluation for a Brownian path. Trans. Amer. Math. Soc. 288, 679-699.

[90] S. J. Taylor and A. A. Watson (1985), A Hausdorff measure classification of polar sets for the heat equation. Math. Proc. Camb. Philos. Soc. 97, 325-344.

[91] F. Testard (1986), Polarité, points multiples et géométrie de certain processus gaussiens. Publ. du Laboratoire de Statistique et Probabilités de l'U.P.S. Toulouse, 01-86.

[92] C. Tricot (1982), Two definitions of fractional dimension. Math. Proc. Camb. Philos. Soc. 91, 57-74.

[93] C. A. Tudor and Y. Xiao (2007), Sample path properties of bifractional Brownian motion. Bernoulli 14, 1023-1052.

[94] J. B. Walsh (1986), An introduction to stochastic partial differential equations. École d'été de probabilités de Saint-Flour, XIV-1984, 265-439, Lecture Notes in Math., 1180, Springer, Berlin.

[95] W. Wang (2007), Almost-sure path properties of fractional Brownian sheet. Ann. Inst. H. Poincaré Probab. Statist. 43, 619-631.

[96] D. Wu and Y. Xiao (2006), Fractal properties of random string processes. IMS Lecture NotesMonograph Series-High Dimensional Probability. 51, 128-147.

[97] D. Wu and Y. Xiao (2007), Geometric properties of fractional Brownian sheets. J. Fourier Anal. Appl. 13, 1-37.

[98] Y. Xiao (1995), Dimension results for Gaussian vector fields and index- $\alpha$ stable fields. Ann. Probab. 23, 273-291.

[99] Y. Xiao (1996a), Hausdorff measure of the sample paths of Gaussian random fields. Osaka J. Math. 33, 895-913.

[100] Y. Xiao (1996b), Packing measure of the sample paths of fractional Brownian motion. Trans. Amer. Math. Soc. 348, 3193-3213.

[101] Y. Xiao (1997a), Hausdorff measure of the graph of fractional Brownian motion. Math. Proc. Camb. Philos. Soc. 122, 565-576.

[102] Y. Xiao (1997b), Hölder conditions for the local times and the Hausdorff measure of the level sets of Gaussian random fields. Probab. Theory Relat. Fields 109, 129-157.

[103] Y. Xiao (1997c), Packing dimension of the image of fractional Brownian motion. Statist. Probab. Lett. 33, 379-387.

[104] Y. Xiao (1999), Hitting probabilities and polar sets for fractional Brownian motion. Stoch. Stoch. Rep. 66, 121-151.

[105] Y. Xiao (2003), The packing measure of the trajectories of multiparameter fractional Brownian motion. Math. Proc. Camb. Philos. Soc. 135, 349-375.

[106] Y. Xiao (2004), Random fractals and Markov processes. In: Fractal Geometry and Applications: A Jubilee of Benoit Mandelbrot, (M. L. Lapidus and M. van Frankenhuijsen, editors), pp. 261-338, American Mathematical Society.

[107] Y. Xiao (2006), Properties of local nondeterminism of Gaussian and stable random fields and their applications. Ann. Fac. Sci. Toulouse Math. XV, 157-193.

[108] Y. Xiao (2007), Strong local nondeterminism and sample path properties of Gaussian random fields. In: Asymptotic Theory in Probability and Statistics with Applications (T.-L. Lai, Q.-M. Shao and L. Qian, eds), pp. 136-176, Higher Education Press, Beijing. 
[109] Y. Xiao (2008), Spectral conditions for strong local nondeterminism of Gaussian random fields. In Preparation.

[110] Y. Xiao and T. Zhang (2002), Local times of fractional Brownian sheets. Probab. Theory Relat. Fields 124, 204-226.

[111] A. M. Yaglom (1957), Some classes of random fields in $n$-dimensional space, related to stationary random processes. Th. Probab. Appl. 2, 273-320.

Yimin XiaO. Department of Statistics and Probability, A-413 Wells Hall, Michigan State University, East Lansing, MI 48824, U.S.A.

E-mail: xiao@stt.msu.edu

URL: http://www.stt.msu.edu/ xiaoyimi 